\title{
DESIGN AND IMPLEMENTATION OF A CODE- PHASE-SHIFT KEYING SPREAD SPECTRUM RECEIVER EMPLOYING A FPGA BASEBAND DECODER
}

\author{
by \\ SUNNY KIN SUN CHAN \\ B.A.Sc.(E.E.), University of British Columbia, 1994 \\ A THESIS SUBMITTED IN PARTIAL FULFILLMENT OF \\ THE REQUIREMENTS FOR THE DEGREE OF \\ MASTER OF APPLIED SCIENCE \\ in \\ THE FACULTY OF GRADUATE STUDIES \\ DEPARTMENT OF ELECTRICAL ENGINEERING
}

We accept this thesis as conforming to the required standard

THE UNIVERSITŸ OF BRITISH COLUMBIA

April 1997

(C) Sunny Kin Sun Chan, 1997 
In presenting this thesis in partial fulfilment of the requirements for an advanced degree at the University of British Columbia, I agree that the Library shall make it freely available for reference and study. I further agree that permission for extensive copying of this thesis for scholarly purposes may be granted by the head of my department or by his or her representatives. It is understood that copying or publication of this thesis for financial gain shall not be allowed without my written permission.

Department of Electrical Engineering

The University of British Columbia Vancouver, Canada

Date Apr.29,97 


\begin{abstract}
Code-phase-shift-keying (CPSK) is a novel direct sequence spread spectrum (DS/SS) signaling technique aimed at improving the data throughput of a DS/SS system without the lost of processing gain. To further evaluate its practical performance, a CPSK modem needs to be designed and implemented. This thesis addresses the above objective, concentrating on the digital receiver.
\end{abstract}

The CPSK receiver consists of three major modules, the IF demodulator, the CPSK decoder, and the PC interface. As with other DS/SS systems, carrier phase and PN code phase synchronization are the two most important issues to be addressed in the design of the CPSK receiver. Carrier phase synchronization is solved by employing a Costas loop in the demodulator to lock the carrier phase and limit the phase uncertainty to either 0 or 180 degree. The uncertainty is then completely resolved by using double thresholds in the CPSK decoder. A modified double dwell serial search scheme with a tracking loop is employed in the CPSK decoder to achieve PN code phase synchronization. Furthermore, to add the flexibility in decoding data with different symbol size and PN code length, a re-programmable Xilinx 4010 FPGA chip is employed to build the decoder.

To evaluate the performance of the receiver, extensive symbol-error-rate (SER) measurements in the presence of additive white Gaussian noise and jamming from another CPSK transmitter are conducted, and the resulting bit error rate (BER), versus signal to noise ratio curves are compared with those of an ideal receiver. Results show that there were 0.25 to $0.85 \mathrm{~dB}$ BER degradation when the carriers of both the transmitter and receiver are perfectly synchronized, and a further $1 \mathrm{~dB}$ degradation when the Costas loop is employed. Jamming from another 
transmitter results in substantial degradations in the BER performance in a noisy environment.

The scalability of the CPSK decoder is also studied based on the reconfigurability of the XC4000 FPGA family. The possible symbol size / PN code length combinations of the decoder that can be fitted into the FPGA chip are discussed and the modifications needed for each major component of the decoder are outlined. Finally, the maximum throughput possible with the FPGAs is addressed. 


\section{Table of Contents}

Abstract

List of Tables

List of Figures

Acknowledgment $\quad$ x

Chapter 1 Introduction $\quad$ - 1

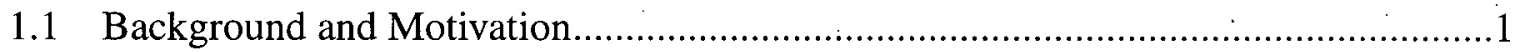

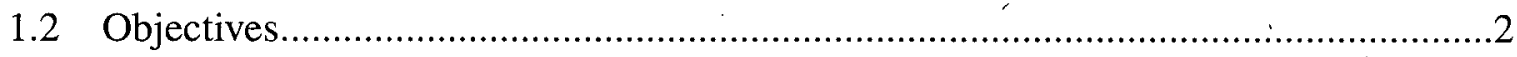

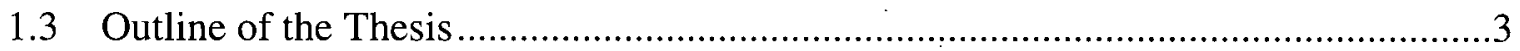

Chapter 2 The Principle of Coded Phase Shift Keying Communication System 4

2.1 Conventional BPSK Direct Sequence Spread Spectrum Communication System.....4

2.2 The Code-Phase-Shift Keying (CPSK) Signaling Method ................................... 8

$\begin{array}{lll}\text { Chapter } 3 & \text { Receiver Design } & 12\end{array}$

3.1 The Requirement Specifications of the CPSK Receiver .....................................12

3.1.1 The System Model .................................................................................. 12

3.1.2 Functional Requirements ........................................................... 13

3.2 High Level Design Considerations .............................................................. 13

3.2.1 The Design Constraints ...................................................................... 14

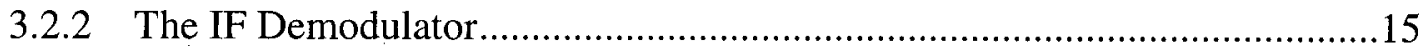

3.2.3 The CPSK Decoder...................................................................... 15

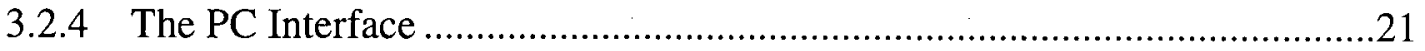

$\begin{array}{lll}\text { Chapter } 4 \text { The Receiver Implementation } & 22\end{array}$

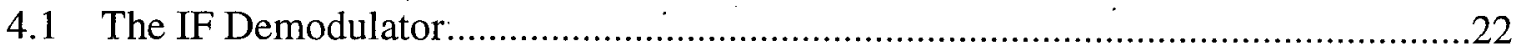




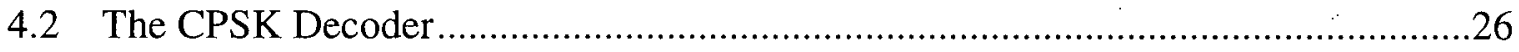

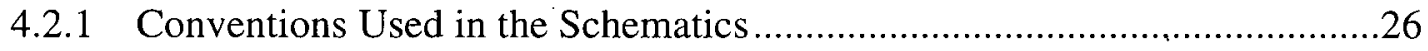

4.2.2 The Digital Correlator and the Threshold Device...................................29

4.2.3 The PN Code Generator with Shift Register.............................................34

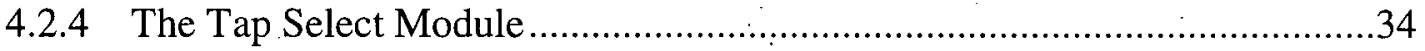

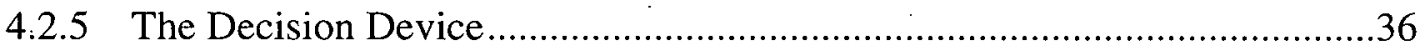

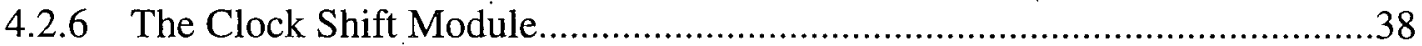

4.2.7 The Search Lock Logic Module .........................................................40

4.2.8 The Tracking Loop .......................................................................40

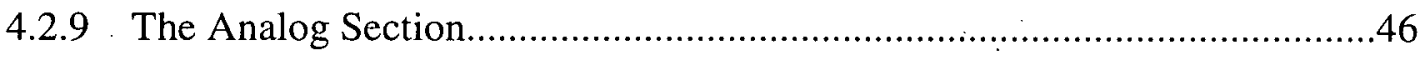

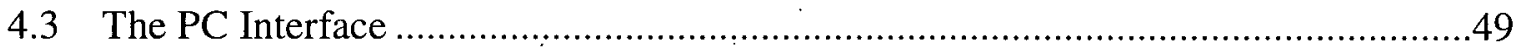

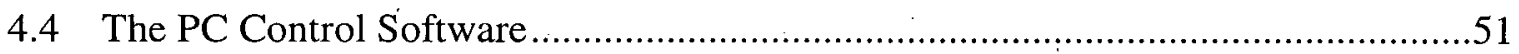

Chapter 5 Testing and Performance Evaluation $\quad 54$

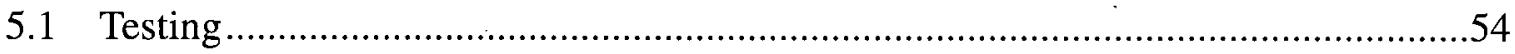

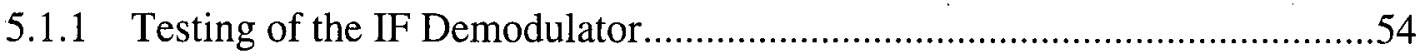

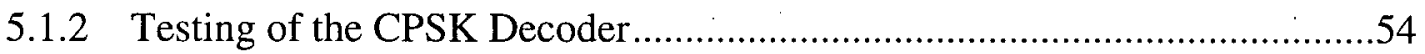

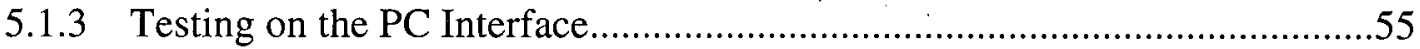

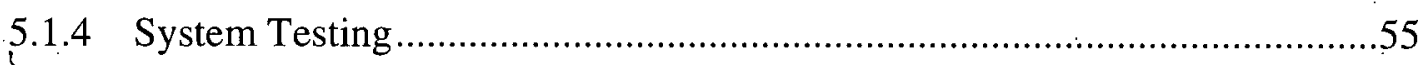

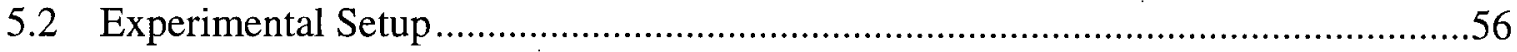

5.3 Performance of the CPSK Receiver Under AWGN ............................................57

5.3.1 Performance with Perfectly Synchronized Carriers...................................58

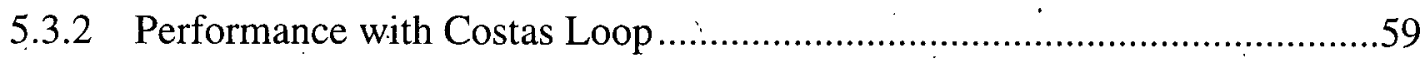

5.4 Performance of the CPSK Receiver With Jamming From Another CPSK Transmitter. 
Chapter 6 Design Scalability of the FPGA Based Decoder

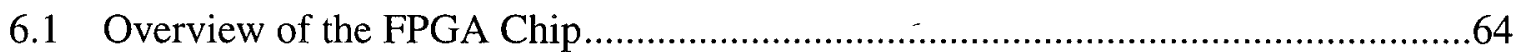

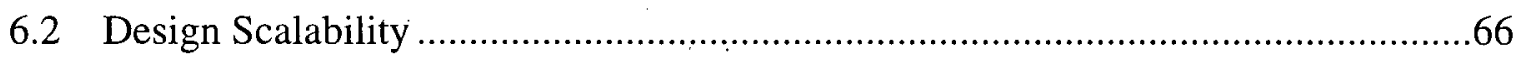

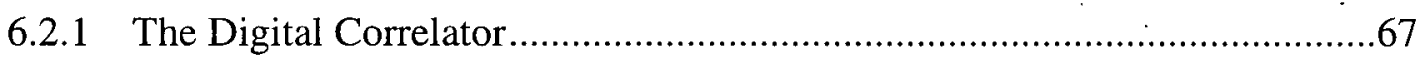

6.2.2 The PN Code Generator with Shift Register.........................................68

6.2.3 The Tap Select Module ....................................................................68

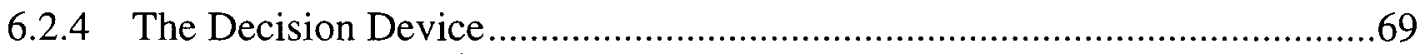

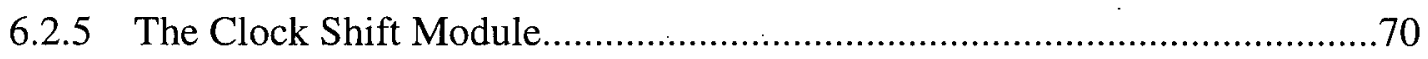

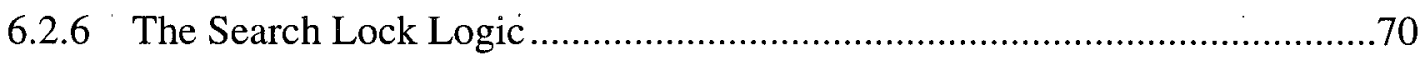

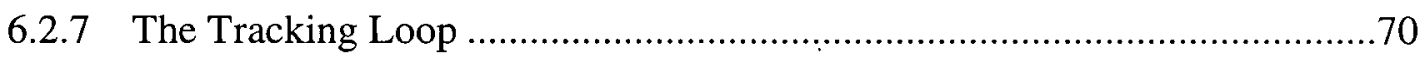

6.2.8 Possible Implementation on Xilinx 4000 FPGA Family ...........................71

6.3 The Maximum Throughput of the FPGA Based Decoder ................................... 72

$\begin{array}{lll}\text { Chapter } 7^{`} \text { Conclusions } & 74\end{array}$

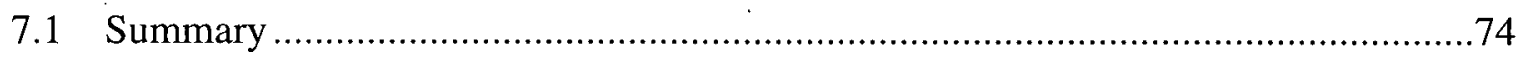

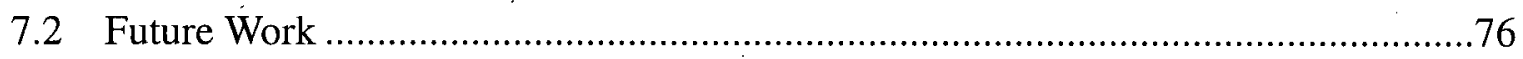

Bibliography $\quad \succ \quad 77$

Appendix A. The Pin Assignment of the FPGA Decoder Chip 79

Appendix B. The Circuit for Configuring and Interfacing the FPGA Decoder Chip : 82

$\begin{array}{lll}\text { Appendix C. The Source Code for the PC Interface } & 84\end{array}$

Glossary $\quad 89$ 


\section{List of Tables}

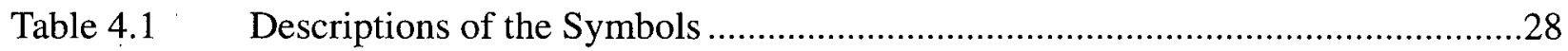

Table 4.2 Digital Representations of Incoming Signal ................................................

Table 4.3 Digital Representations of PN Sequence .........................................................30

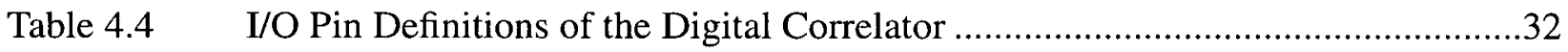

Table 4.5 Representation of the Search Lock States........................................................40

Table 4.6 I/O Pin Definitions of the 8-bit-3-stage Shift Register ................................42

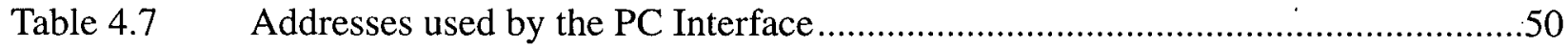

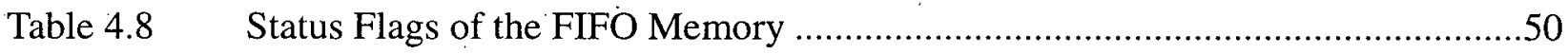

Table 6.1 Size of Accumulator Needed According to G .............................................68

Table 6.2 . Possible Values of the Sum of PN Codes.....................................................71 


\section{List of Figures}

Figure 2.1 Conventional BPSK-DS-SS System

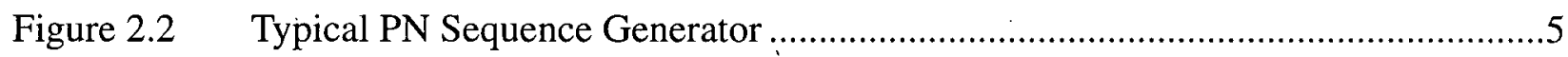

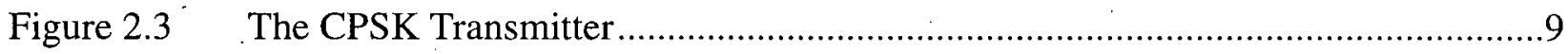

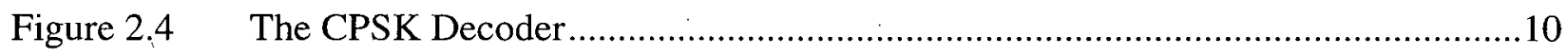

Figure $3.1 \quad$ Block Diagram of a CPSK Communication System .........................................12

Figure 3.2 High Level Design of the IF Demodulator ........................................................16

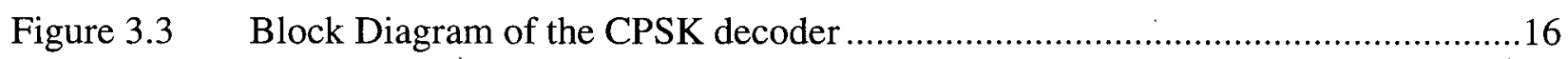

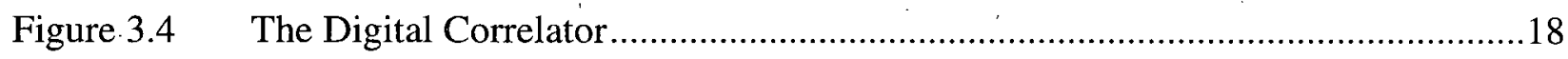

Figure 3.5 State Diagram of the Double Dwell Search Lock Scheme ....................................20

Figure 3.6 Block Diagram of the PC Interface

Figure 4.1 The BPSK Demodulation Section of the IF Demodulator.....................................22

Figure 4.2 The Filter and Amplifier Section of the IF Demodulator .....................................23

Figure 4.3 The Costas Loop Section of the IF Demodulator ………......................................24

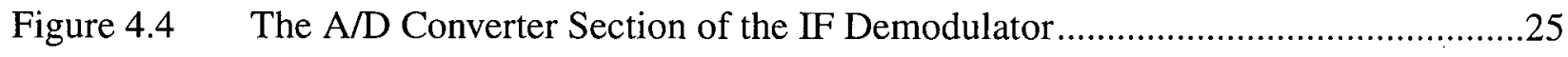

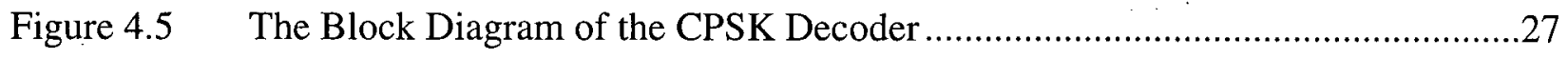

Figure 4.6 Symbols and I/O Conventions Used in the Schematics Composed with Cadence

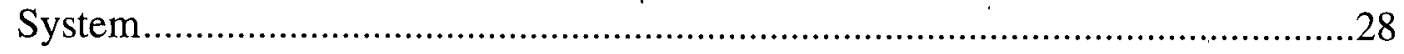

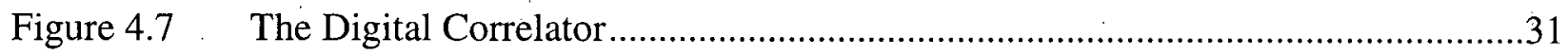

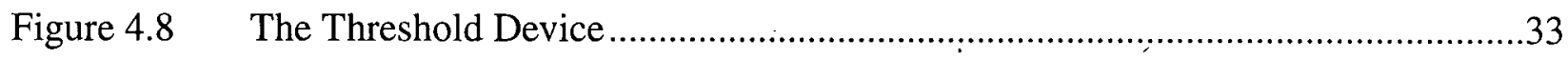

Figure 4:9 Correlations of Incoming Signal with Local PN Code ..........................................33

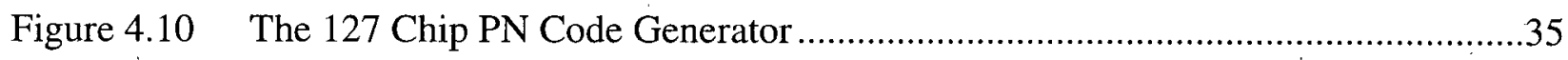

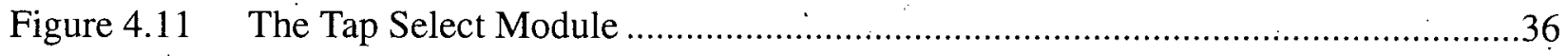


Figure 4.12 The Decision Device

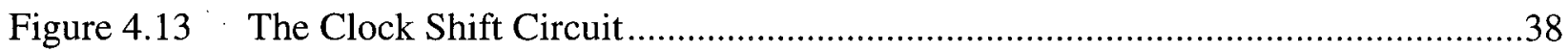

Figure 4.14 The Schematic of the Search Lock Logic Circuit ...............................................41

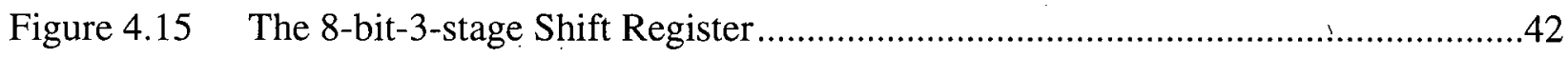

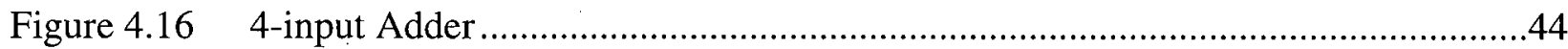

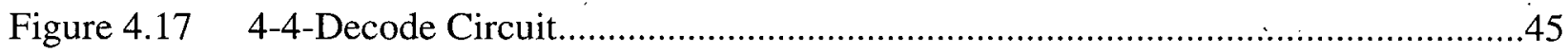

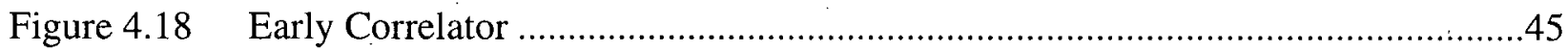

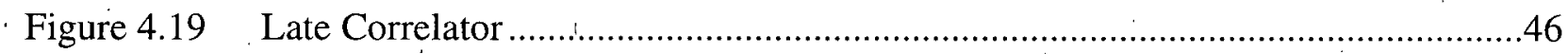

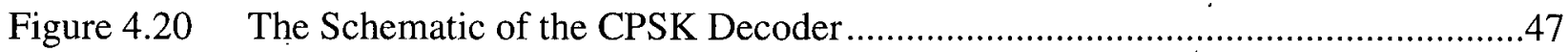

Figure 4.21 The Analog Section of the CPSK Decoder.........................................................

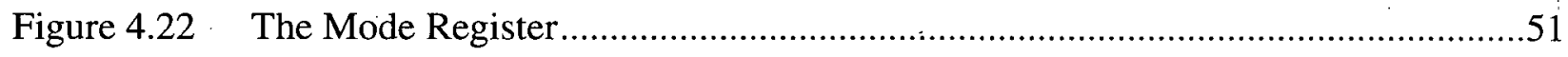

Figure 4.23 The Schematic of the PC Interface …….......................................................52

Figure 4.24 The Flow Chart of the PC Control Software......................................................53

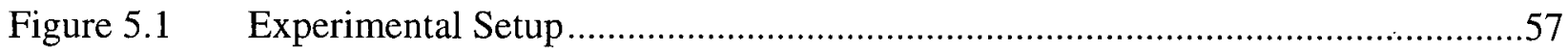

Figure 5.2 Performance of the Receiver with Synchronized Carrier .....................................60

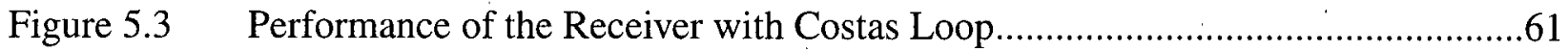

Figure 5.4 Performance of the Receiver With Jamming From Another Transmitter ..............62

Figure 5.5 Costas Loop Demodulator With Signals From Both Transmitters ........................63

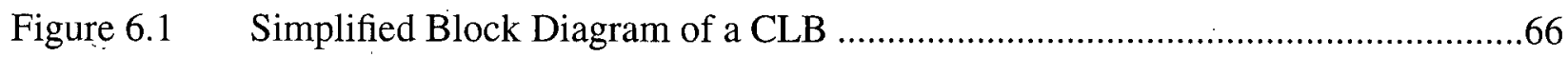

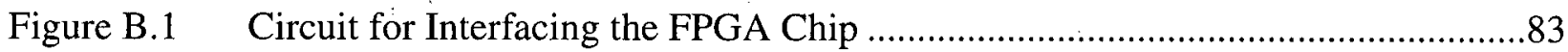




\section{Acknowledgment}

I would like to express my deepest gratitude to my supervisor, Dr. Victor C. M. Leung for the direction and numerous advice he gave throughout the whole thesis research. I would like to acknowledge the Science Council of British Columbia for the financial assistance.

Special thanks go to Mr. Hansen Wang for his help in the design and implementation of the RF demodulator with the Costas loop, Mr. Mark Jay McCutcheon for his advice in digital circuit design with Cadence, and Mr. Robert Link for his design and implementation of the CPSK transmitter, and the assistance in circuit testing and performance measurement.

I would also like to thank my colleagues, my, family members, ánd my friends in Richmond Chinese Alliance Church for they have given me numerous and consistent supports.

Last but not least, thank to God who have guided me throughout the research. Without the strength from Him, I would not be able to finish the research. 


\section{Chapter 1 Introduction.}

In this introductory chapter, the background and motivation of the thesis research and the objectives are discussed. The outline of the thesis is also shown in the last section of this chapter.

\subsection{Background and Motivation}

As technology advances, the cost of manufacturing components for spread spectrum (SS) communication systems has been reduced dramatically and thus SS systems for non-military use become economically feasible. A spread spectrum communication system can be simply defined as a communication system having a bandwidth which is larger than the minimum bandwidth required to transmit the information [1]. In such a system, the information is spread to a wider bandwidth at the transmitter and at the receiver, the signal is restored to the original bandwidth. SS signalling schemes are particularly suitable for wireless local area network (WLAN) applications for they have the following properties:

- Antijamming,

- High interference immunity,

- Information hiding, and

- Capable of having multiple channels within the same bandwidth (code division multiple access).

Currently, wireless transceivers available in the market which employ conventional direct sequence spread spectrum (DS/SS) signaling scheme provide a data throughput up to $2 \mathrm{Mbits} / \mathrm{sec}$ which is limited by the available frequency bandwidth. The processing gain defined by the ratio of bandwidth after and before the signal is spread by a code sequence, is an important parameter in 
evaluating the performance of a SS system. Higher processing gain results in higher interference immunity. However, the data throughput is decreased relatively. One way to improve the throughput is to use M-ary SS scheme in which several different pseudo noise (PN) sequences are used to send several data bits over the communication channel at a time. However, this scheme has a drawback in that the PN codes may interfere with each other and depending on the operating environment, the bit error rate (BER) performance may only have little improvement. Although orthogonal maximal length PN sequences can be used, the number of available sequences for the same code length is limited. The other drawback includes the need of extra hardware to generate different PN codes. To minimize these drawbacks, a novel M-ary SS signalling scheme, known as code-phase-shift keying was proposed (CPSK) [4]. From the simulations and implementation using DSP techniques done by previous research works, the CPSK scheme was proved to be practical with enhanced data throughput as compared with conventional DS/SS schemes[4][5]. However, the cost of building a CPSK transceiver (or modem) using DSP boards is too expensive and the data throughput is too low if it is to be commercialized. Thus, an alternative approach to build a cost reduced CPSK modem is necessary.

\subsection{Objectives}

The objectives of this thesis are the design, implementation, and evaluation of a CPSK receiver using VLSI techniques to make it more economically feasible for commercialization. With some appropriate software, the final product will be able to replace conventional wired LAN cards in PCs with the additional benefit of mobility. Also, the flexibility in changing system parameters such as the PN sequence length and the word size is considered in designing the receiver to enable it to adapt to different envíronments. 


\subsection{Outline of the Thesis}

The remainder of this thesis is written in 6 chapters. Chapter 2 describes the basic principles of CPSK signaling and the compares it with conventional DS/SS schemes. In Chapter 3, the high level design of the CPSK receiver, including the system model and the module break-down of the system, is described. Then, a detailed explanation of the design and implementation of the actual circuits of the receiver is presented in Chapter 4. Chapter 5 is devoted to the testing and performance evaluation of the CPSK receiver. The testing methodology and BER measurements with and without jamming from another CPSK transmitter are presented. Since the heart of the CPSK decoder is a FPGA (field programmable gate array) chip manufactured by Xilinx, the limits of the chip and its ability to accommodate different configurations of the CPSK decoder needs to be addressed and they are discussed in Chapter 6. Finally, in Chapter 7, the thesis is concluded with a summary of work and suggestions for future developments. 


\section{Chapter 2 The Principle of Coded Phase Shift Keying Com- munication System}

In this chapter, a brief discussion of a conventional DS/SS communication system is presented which is followed by an explanation of the CPSK signalling scheme. The comparison of the performance of the CPSK signaling scheme with that of a conventional DS/SS signaling scheme is also discussed.

\subsection{Conventional BPSK Direct Sequence Spread Spectrum Communication System}

Figure 2.1 shows a simplified conventional BPSK DS/SS communication system. The data with rate $R_{d}$ to be sent is spread by a pseudonoise $(P N)$ sequence running at a rate $R_{c}$ [2]. Then the spreading factor or the processing gain of the system is the ratio $G=R_{c} / R_{d}$. Assume that. the bandwidth of the PN sequence is much larger than that of the data, the resulting transmit bandwidth is approximately the bandwidth of the PN sequence. The spread signal is then BPSK modulated and is sent over the communication channel to the receiver. At the receiver, assume

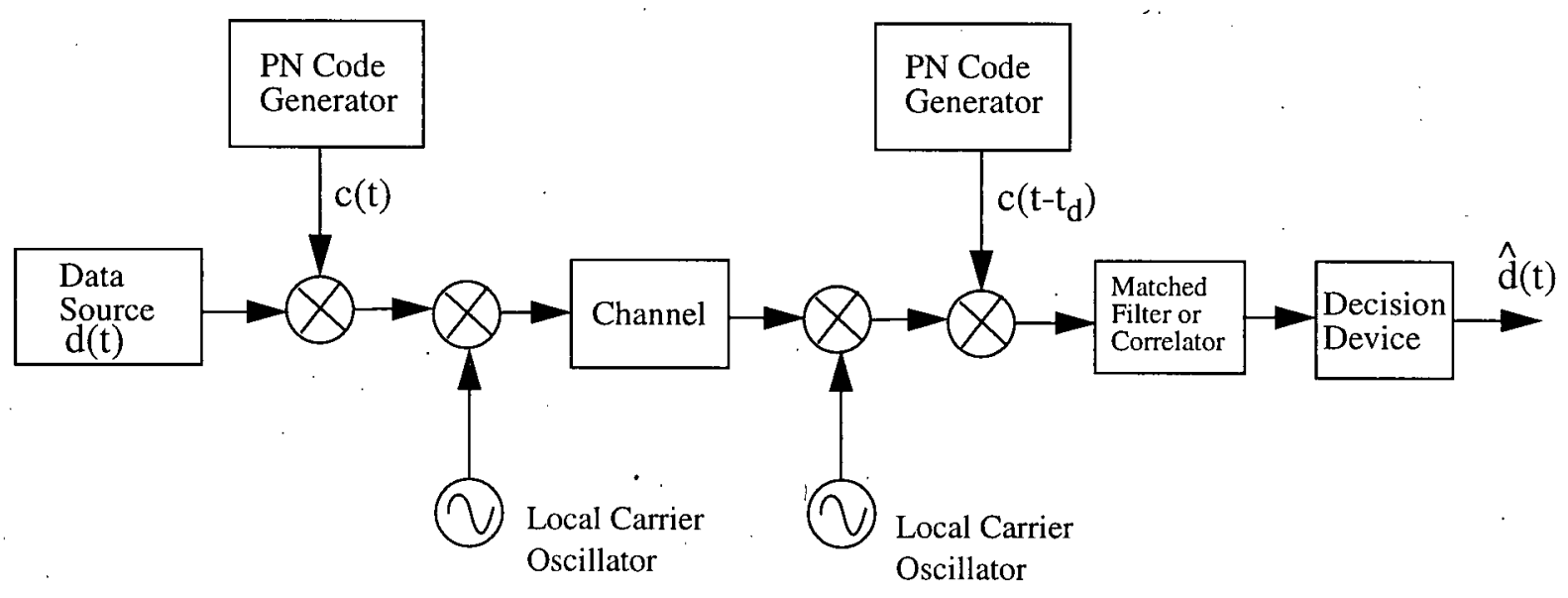

Figure 2.1 Conventional BPSK-DS-SS System . 
carrier and spreading synchronization is made, the signal is first demodulated by a BPSK demodulator and finally restored to the original signal by a despreader. The despreader is essentially a multiplier that multiplies the signal with the same synchronized PN sequence as that in the transmitter. The bandwidth of the signal is restored and the data is decoded [3]. As will be seen in the following explanation, the BPSK-DS/SS system offers a high rejection of jamming signals.

Mathematically, the data to be sent is represented by a bit-stream d(t) which has either +1 or -1 as its value. A PN sequence $\mathrm{c}(\mathrm{t})$, usually generated by a linear-feedback-shift-register such as the one shown in Figure 2.2, is used to spread the signal [1]. The chip duration or the pulse width is $T_{c}=T_{b} / G$ where $T_{b}$ is the bit duration of the data stream. At the transmitter output, the signal is represented by:

$$
x_{c}(t)=A d(t) c(t) \cos \left(\omega_{c} t\right)
$$

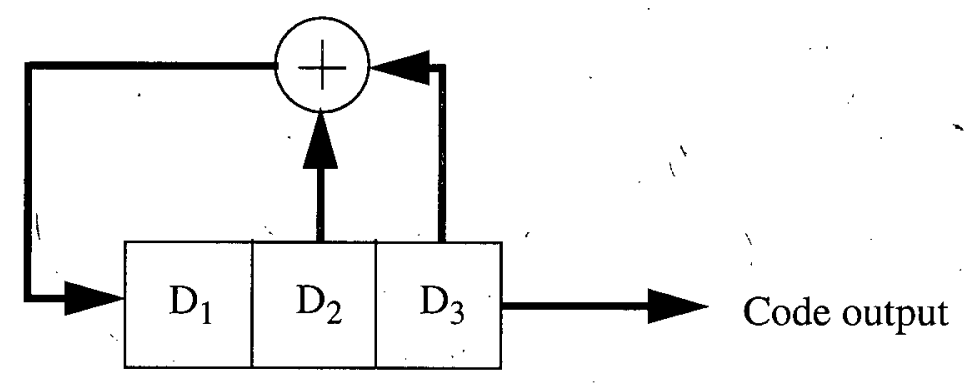

Three-stage maximal generator

Figure 2.2 Typical PN Sequence Generator

Assuming that there are additive white Gaussian noise (AWGN) $n(t)$ and narrow-band 
interference $j(t)$ in the channel, the received signal at the receiver input is:

$$
z(t)=x_{c}(t)+n(t)+j(t)
$$

After the received signal is BPSK demodulated and the double frequency terms are filtered, the signal becomes:

$$
z_{1}(t)=A d(t) c(t) c(t-\Delta)+n^{\prime}(t) c(t-\Delta)+j^{\prime}(t) c(t-\Delta)
$$

where

$$
\begin{aligned}
n^{\prime}(t) & =2 n(t) c(t) \cos \left(\omega_{c} t\right) \\
j^{\prime}(t) & =2 j(t) c(t) \cos \left(\omega_{c} t\right)
\end{aligned}
$$

$\Delta$ is the misalignment of the locally generated code at the receiver with the code in the received signal. Assuming perfect code synchronization $(\Delta=0)$, after every $\mathrm{T}_{\mathrm{b}}$ seconds, the correlator or matched filter becomes:

$$
z= \pm A T_{b}+N_{j}+N_{g}
$$

where

$$
\begin{aligned}
& N_{j}=\int_{0}^{T_{b}} 2 j(t) c(t) \cos \left(\omega_{c} t\right) d t \\
& N_{g}=\int_{0}^{T_{b}} 2 n(t) c(t) \cos \left(\omega_{c} t\right) d t
\end{aligned}
$$

For single tone jamming, $\mathrm{j}(\mathrm{t})$ can be modeled as:

$$
j(t)=A_{I} \cos \left[\left(\omega_{c}+\Delta \omega\right) t+\phi\right]
$$

where $A_{I}$ is the amplitude of the jamming signal and $\Delta \omega$ is its offset frequency from the carrier frequency. It is assumed that $\Delta \omega<2 \pi / T_{c}$. The means of $\mathrm{Ng}$ and $\mathrm{Nj}$ are zero, and their variances are given by: 


$$
\begin{aligned}
& \operatorname{var}\left(N_{g}\right)=N_{o} T_{b} \\
& \operatorname{var}\left(N_{j}\right)=\frac{T_{c} T_{b}^{2}}{2} .
\end{aligned}
$$

Then the probability of bit error can be shown to be [6]:

$$
P_{E}=\frac{1}{2} \operatorname{erfc}\left(\sqrt{\frac{A^{2} T_{b}^{2}}{2 \sigma_{T}^{t}}}\right)
$$

where

$$
\sigma_{T}^{t}=N_{o} T_{b}+\frac{T_{c} T_{b} A_{I}^{2}}{2}
$$

is the total variance of the noise plus interference components at the integrator output.

Moreover,

$$
\frac{A^{2} T_{b}^{2}}{2 \sigma_{T}^{2}}=\frac{P_{s}}{P_{n}+P_{I} / G}=\frac{S N R}{1+\frac{(S N R)(J S R)}{G}}
$$

where

$P_{s}=A^{2} / 2$ is the signal power at the input

$P_{n}=N_{o} / T_{b}$ is the Gaussian noise power in the bit-rate bandwidth

$P_{I}=A_{I}^{2}$ is the power of the interfering component at the input

$\mathrm{G}=\mathrm{T}_{\mathrm{b}} / \mathrm{T}_{\mathrm{c}}$ is the processing gain of the DS/SS system

$\mathrm{SNR}=\mathrm{P}_{\mathrm{S}} / \mathrm{P}_{\mathrm{n}}$ is the signal-to-noise ratio 
$\mathrm{JSR}=\mathrm{P}_{\mathrm{I}} / \mathrm{P}_{\mathrm{S}}$ is the jamming-to-signal ratio

It is easily seen from the above equations that spreading does not improve the error rate performance of coherent BPSK reception in AWGN but the single tone jamming effect is reduced by a factor of the processing gain $\mathrm{G}[6]$.

To maintain the above performance while obtaining a higher data throughput, M-ary modulation can be used. In such a modulation scheme, $M$ different code sequences representing M different alphabets are used to transmit a data symbol. In the following section, one M-ary modulation scheme, the CPSK scheme, is discussed. CPSK not only offers the performance advantages of conventional $\mathrm{M}$-ary systems, but it provides many implementation advantages as well.

\subsection{The Code-Phase-Shift Keying (CPSK) Signaling Method}

A CPSK transmitter is shown in Figure 2.3. The data are grouped into k-bit data symbols represented by an integer $\mathrm{m}$ where $0 \leq m \leq M-1$. The symbols are used to select one of the $\mathrm{M}=$ $2^{k}$ code sequences $p_{m}(t)=p\left(t-m_{c} T_{c}\right)$ which are phase-shifted by $m_{c}=m(L+1) / M$ where $L$ is the length of the PN sequence with chip interval $\mathrm{T}_{\mathrm{c}}$. Different code sequences are generated by a PN code generator with a code chip delay line. The selected sequence is then up-converted for transmission at carrier frequency $\omega$ and the transmitted signal is:

$$
s(t)=A p_{m}(t) \cos \left(\omega_{c} t\right)
$$

The received signal $r(t)=s(t)+j(t)+n(t)$ at the input of the receiver is fed into a bank of M correlators as shown in Figure 2.4. For a maximal length PN sequence $p(t)$, the autocorrelation function is: 


$$
\Re(\tau)=\sum_{i=0}^{i=L-1} p\left(i T_{c}\right) p\left((i+\tau) T_{c}\right)=\left\{\begin{array}{c}
L \tau=0, L, 2 L, \ldots \\
-1 \text { otherwise }
\end{array}\right.
$$

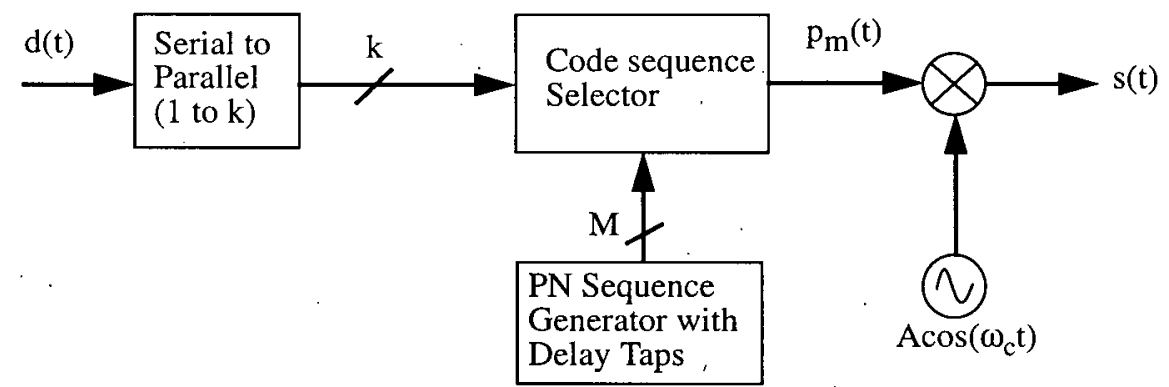

0

Figure 2.3 The CPSK Transmitter

Assume that carrier, chip, and data symbol synchronization are made at the receiver. When the m-th symbol is sent, the normalized output of the m-th correlator at the end of every symbol period is:

$$
z_{m}=1+J_{m}+N_{m}
$$

and the output of the other correlators, $z_{i}, i \neq m$, are:

$$
z_{z}=-\frac{1}{L}+J_{i}+N_{i}
$$

with the noise and interference terms $\forall i \quad 0 \leq i \leq M-1$ :

$$
\begin{aligned}
N_{i} & =\frac{2}{A L} \int_{0}^{T_{s}} n(t) p_{i}(t) \cos (\omega t) d t \\
J_{i} & =\frac{2}{A L} \int_{0}^{T_{s}} j(t) P_{i}(t) \cos (\omega t) d t
\end{aligned}
$$


The decision device locates the correlator which gives the largest output and selects the symbol according to the location of the largest correlator output for parallel-to-serial decoding into the output data.

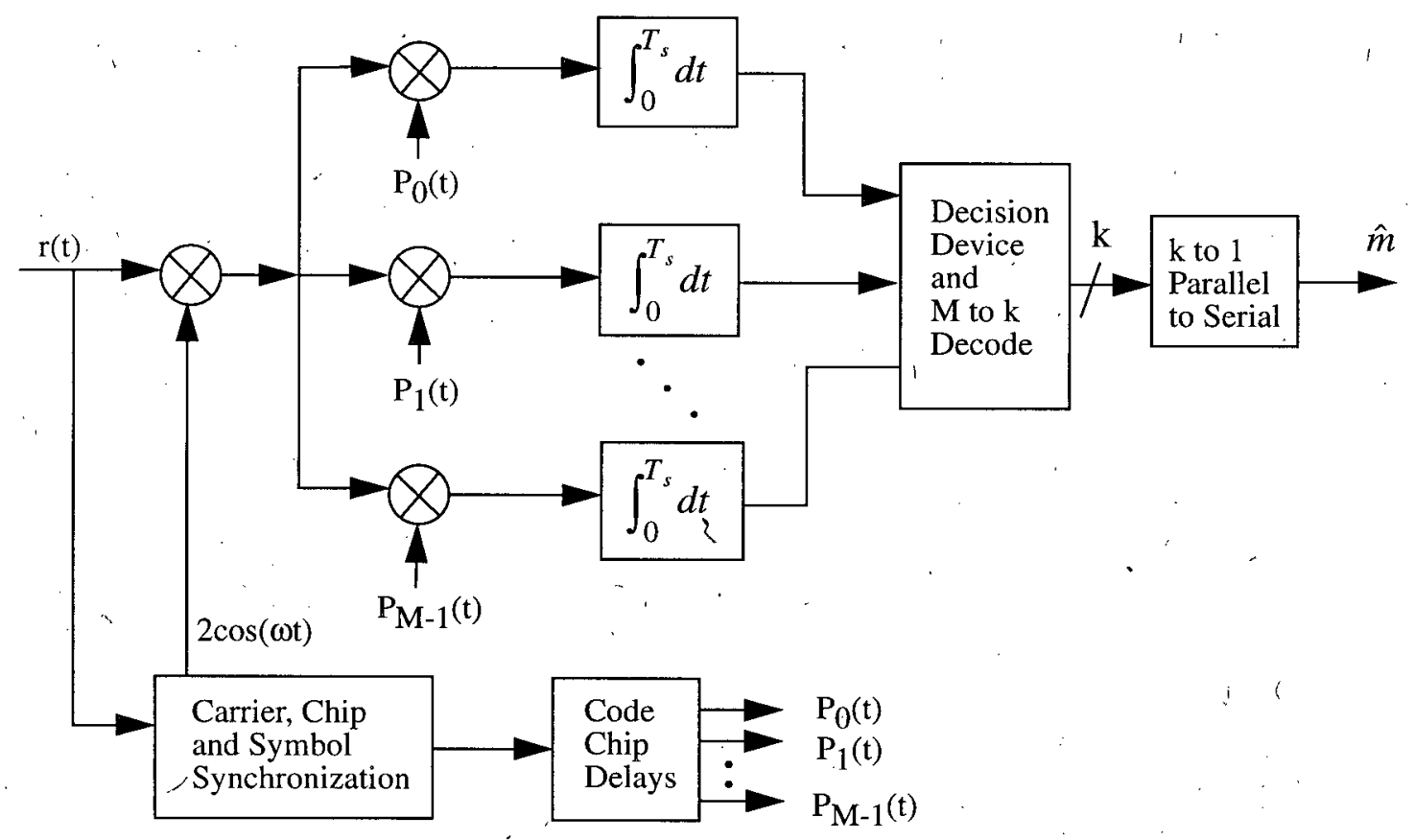

Figure 2.4 The CPSK Decoder

If the interference is a single tone at the carrier frequency,

$$
j(t)=A_{I} \cos (\omega t+\phi)
$$

where $\phi$ is the phase difference between the signal carrier and the jamming signal, then all $\mathrm{J}_{\mathrm{i}}$ 's are the same and therefore the decision are not affected by the carrier-wave jamming signal.

The symbol error rate (SER) in AWGN with zero mean and $E[\mathrm{Ni2}]=\mathrm{No} / 2$ is: 


$$
P_{e}=\frac{1}{\sqrt{2 \pi}} \int_{-\infty}^{\infty}\left\{1-\left(\frac{1}{2} \operatorname{erfc}\left(\frac{y}{\sqrt{2}}\right)\right)^{M-1}\right\} \exp \left(\frac{-\left(y-\sqrt{2\left(E_{s} / N_{o}\right)}\right)^{2}}{2}\right) d y
$$

and the detail calculation of the above error probability can be found in [4].

Also, from the simulation results in [4], the performance of CPSK is similar to M-FSK, in that the power efficiency increases with $\mathrm{M}$. When $k \geq 3$, CPSK requires a lower $E_{b} / N_{o}$ than that required by B-PSK at any given BER. For example, at BER $=10^{-5}, E_{b} / N_{o} \approx 9.5 \mathrm{~dB}$ for BPSK-DS/ SS while $E_{b} / N_{o} \approx 8.3 \mathrm{~dB}, 7.3 \mathrm{~dB} ; 6.7 \mathrm{~dB}$, and $6 \mathrm{~dB}$ with $\mathrm{k}=3,4,5$, and 6 respectively for CPSK. Therefore, at $\mathrm{k}=6$, the power saving for CPSK is $3.5 \mathrm{~dB}$. Moreover, the bandwidth of CPSK is dependent on the spreading gain but not $\mathrm{M}$ and the bandwidth efficiency can be increased by increasing $\mathrm{M}$ (i.e. the number of different code-phases).

In the following chapters, the details of the design, implementation, and the evaluation of the performance of the CPSK modem are discussed. 


\section{Chapter 3 Receiver Design}

The requirement specifications and the high level design of the CPSK receiver are discussed in this chapter.

\subsection{The Requirement Specifications of the CPSK Receiver}

The block diagram of a complete CPSK communication system is shown in Figure 3.1. The focus of this thesis is on the design and implementation of the CPSK receiver which includes an IF demodulator, a CPSK decoder, and a PC interface. The following sub-sections define the requirements and the high level design of the CPSK receiver.

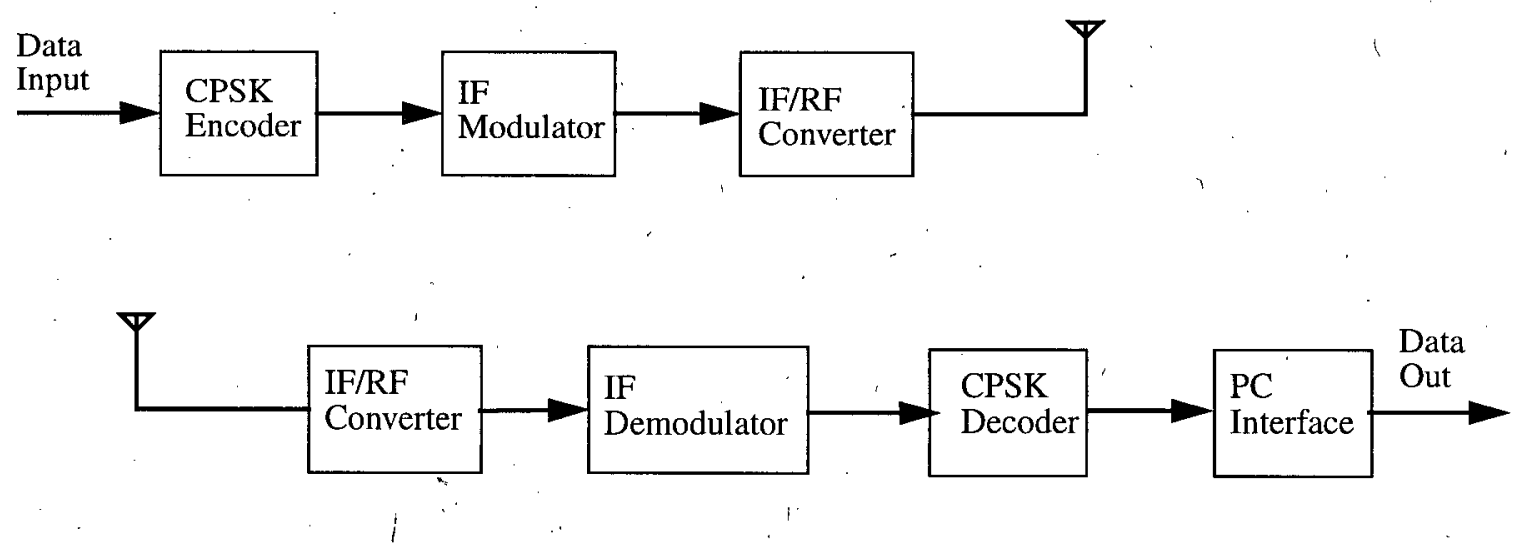

Figure 3.1 Block Diagram of a CPSK Communication System

\subsubsection{The System Model}

The system consists of a CPSK transmitter and a CPSK receiver housed in two separate computers. As shown in Figure 3.1, data are sent to the encoder for CPSK encoding. The encoded signal is then fed into the IF modulator for BPSK modulation and finally up-converted to an RF frequency before it is sent to the receiver. When the transmitted signal is received by the receiver, 
it is down-converted to IF frequency before it is demodulated. The demodulated signal is then decoded by the decoder and finally the decoded data are read into the computer through the PC interface.

\subsubsection{Functional Requirements}

The functional requirements of the CPSK receiver are listed as follows:

1.Encoding/decoding method: CPSK direct sequence spread spectrum signaling scheme

2.IF modulation/demodulation scheme: binary phase shift keying (BPSK)

3.Data format: bit stream (Data packets can be assembled / disassembled by higher layer protocols in the OSI model)

4.Flexibility: The receiver should be able to be reconfigured to decode signals with different PN sequence lengths and word size.

5.Level of integration: The final product should be built with as few discrete components as possible which means that most of the circuits should be put into a VLSI chip or programmable chip so that the cost of the product can be minimized.

\subsection{High Level Design Considerations}

In this section, the choice of the approach in each major part of the receiver is discussed. The actual circuit design and the calculations of some important parameters are discussed in Chapter 4. As mentioned in the requirement specifications, the receiver consists of 3 parts, the IF demodulator, the CPSK decoder, and the PC interface. There are several reasons for splitting the receiver into 3 separate parts: Each part is responsible for processing the signal at different signalling levels and the implementation requirements are different. The IF demodulator demodulates 
the input signal with carrier frequency of $140 \mathrm{MHz}$. Discrete components and a well designed PCB are needed to ensure maximum shielding and minimum noise generation within the circuit. The CPSK decoder despreads the digitized signal from the RF demodulator and a vast amount of digital logic components are needed. The best way to implement the decoder is to use VLSI techniques to build a custom chip (either an ASIC or a re-programmable chip). The PC interface collects the decoded data from the CPSK decoder and delivers the data to the PC, it must be built into an AT card so that it can be installed in the PC. As the design is not too complex, discrete logic components and a multi-purpose AT card were used to build the interface. Priority in design was given to modularity, simplicity, low cost, and reliability.

\subsubsection{The Design Constraints}

Some more detailed and design dependent specifications are determined by the constraint that the receiver has to communicate with the DSP based CPSK transmitter already implemented in previous research [5]. Most of the detailed specifications of the receiver are adapted from that of the transmitter as follows:

1. Carrier frequency: $140 \mathrm{Mhz}$

2. $P N$ code chip rate $R_{\mathrm{c}}: 1 \mathrm{MHz}$

3. PN code length L: 127 chips

4. Input range to the CPSK decoder: $0-2 \mathrm{~V}$

5. Resolution of the input signal to the CPSK decoder: 8 bit unsigned and level-shifted

6. Data symbol size S: 3 bit

From the above constraints, the data rate $\mathrm{R}_{\mathrm{d}}$ is given by: 


$$
R_{d}=S \times \frac{R_{c}}{L}=23.62 \mathrm{kbps}
$$

The data rate is limited by the fact that $\mathrm{Rc}$ is determined by the transmitter previously implemented based on a DSP board. If we have another transmitter that is capable of providing a higher chip rate, the data rate could be improved. In Chapter 6, the limit of the data that can be achieved by using FPGA chips is explored. The reason for choosing a 127-chip PN code is that there are more choices for different PN codes at this length so that the receiver can be easily modified to decode signal spread with different PN codes for CDMA implementations in future development.

\subsubsection{The IF Demodulator}

The high level design of the IF demodulator is shown in Figure 3.2. The incoming signal from the transmitter is product modulated to give in phase (I) and quadrature (Q) baseband signals. To ensure coherent reception, a Costas loop is included to synchronize the local oscillator (LO) to the carrier frequency [10]. However, there is still a 180 degree phase uncertainty in the demodulated baseband signal, but this can be resolved in the CPSK decoder as seen in Chapter 4. Finally, the I and Q baseband signals are A/D converted for decoding by the CPSK decoder. The detail circuit as well as the implementation are shown in Chapter 4. Since the demodulator involves analog and digital sections, discrete components are used and the whole circuit is implemented on a single circuit board.

\subsubsection{The CPSK Decoder}

The CPSK decoder contains four major parts, the despreader, the synchronization and tracking device, the decision device, and the data reassembler, as shown in Figure 3.3. The 


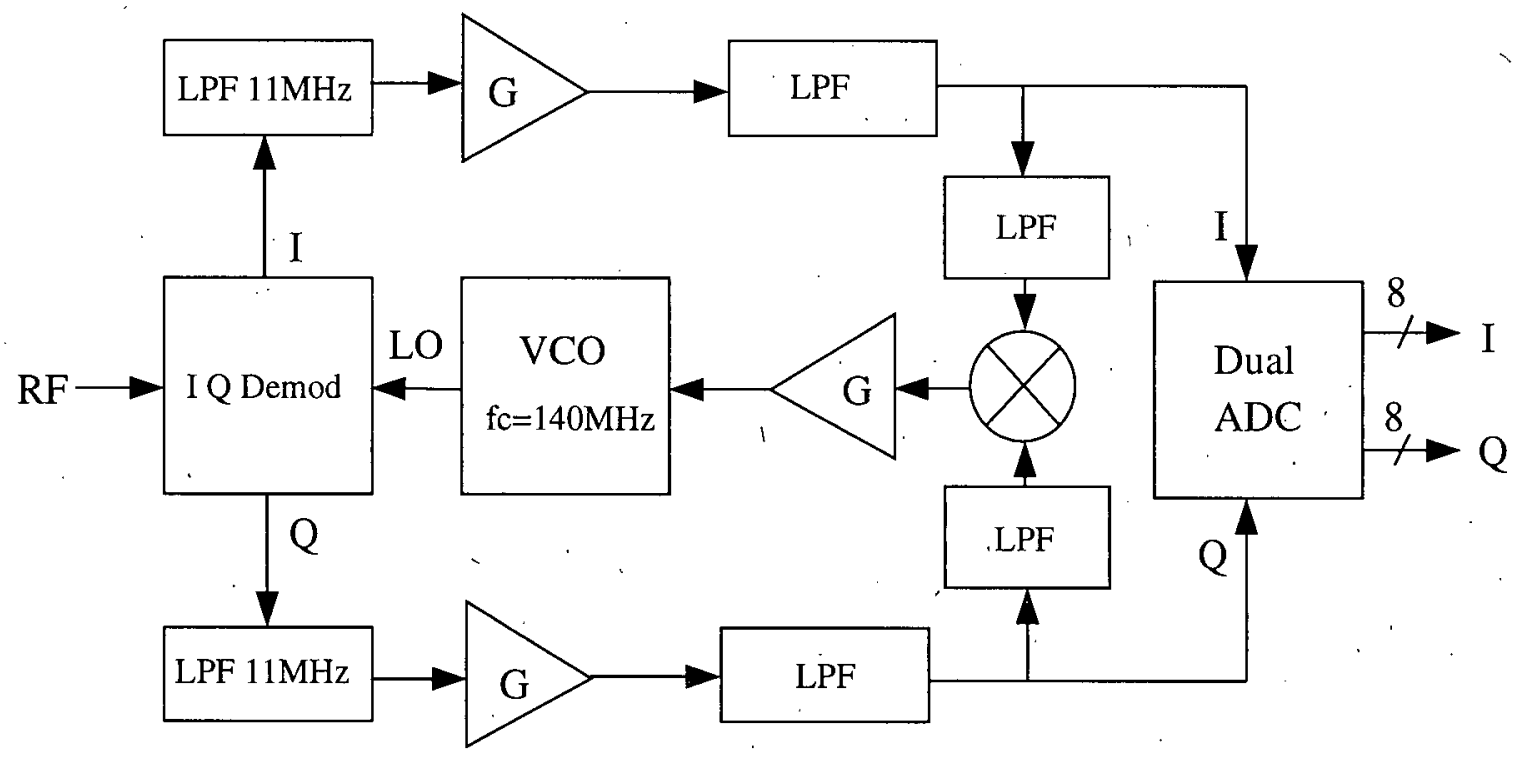

Figure 3.2 High Level Design of the IF Demodulator

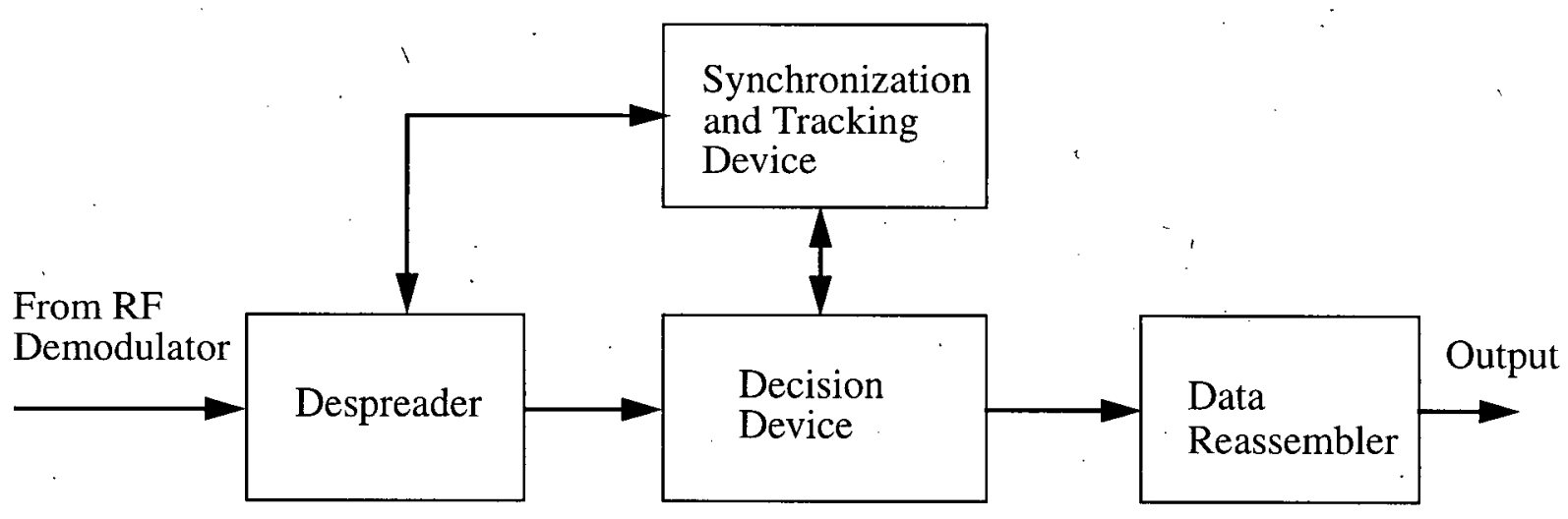

Figure 3.3 Block Diagram of the CPSK decoder

despreader is used to despread the signal by multiplying the incoming signal with phase shifted versions of the PN code and accumulating the value for a whole PN code period. The result is fed into the decision device for estimating the amount of phase shift of the incoming signal with respect to the reference local PN code. The data reassembler then looks up the symbol from the 
amount of phase shift detected and sends out the symbol. Before the data can be decoded, the received signal needs to be synchronized with the receiver's local PN code. The need for synchronization and the steps involved in the process are explained as follows. The PN codes of both the transmitter and receiver are driven by separate clock signals and so the phase of both PN codes are not synchronized. In oder to despread the received signal, the phase of the local PN code must align with that of the received signal. The alignment is done in 2 stages, namely acquisition (or initial synchronization) and tracking. In acquisition stage, the local PN codes is sliding half a chip every code cycle until the code phase difference with the received PN code is no more than half a chip. Then, the alignment is fine-tuned in tracking stage by slowly adjusting the clock that generate the local PN code so that maximum correlation is always maintained. The synchronization and tracking device is responsible for the phase alignment.

More in-depth discussions about each module are presented in the following subsections.

\subsubsection{The Despreader}

There are 2 approaches to despread the incoming signal with different phase shifted versions of the local PN code. One is the matched filter approach and the other is the correlator approach. The matched filter approach employs a band of summers with the number of inputs corresponding to nearly twice the length of the pseudo random sequence [7]. Although the structure of the summers are identical, the number of gates involved is huge. For example, if the code length is 127 , then there are approximately 127 summers and each summer contains 127 half adders and 191 full adders. The total number of gates involved will be approximately 513,920. Therefore this approach is impossible if all the summers are to be built in one programmable chip such as the largest one from Xilinx which contains as many as 62000 gates [19]. The other 
approach is to use digital correlators instead of matched filters. The correlator is basically a product modulator with an accumulator. The product modulator is an XNOR gate which is used to despread the incoming signal from the RF demodulator. The accumulator is used to sum up and store the despread signal. In this approach, the number of gates for a correlator is approximately 242 and the total number of gates needed to implement the bank of correlators will be approximately 15246 gates for a PN sequence of length 127 . Therefore, this approach is more feasible. The block diagram for the correlator is shown in Figure 3.4.

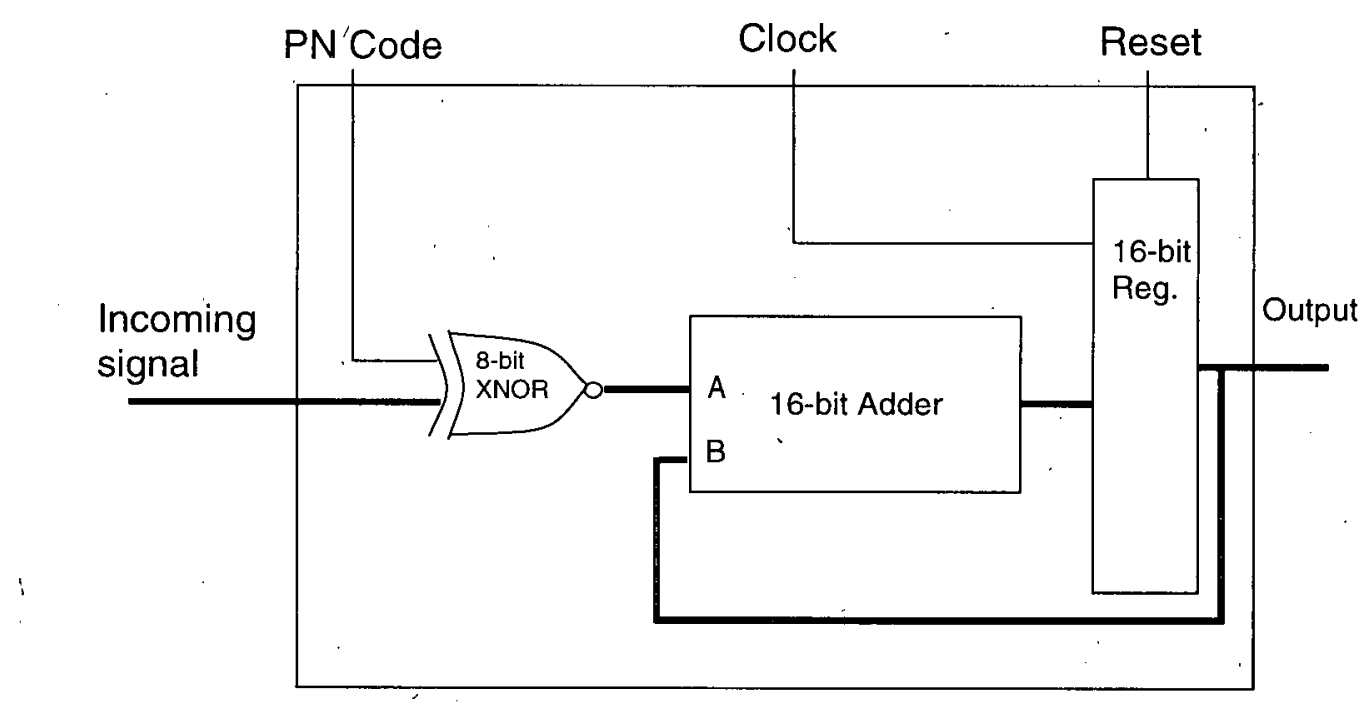

Figure 3.4 The Digital Correlator

\subsubsection{The Synchronization and Tracking Device}

The device consists of 2 parts: The acquisition part and the tracking part. The acquisition part is used for initial synchronization when a preamble containing pure zeros (the PN sequences with zero phase shift) is being sent. The length in the preamble is usually equal to the PN code length to ensure the acquisition is made. However, since a bank of correlators (as described in the 
next paragraph) is used to decode the data, the preamble length and the average acquisition time required will be reduced by a factor equal to the number of correlators used. After acquisition is made, the tracking circuit takes over to monitor and adjust for any phase changes while decoding the received signal. The transition between acquisition state and tracking state is controlled by a search lock logic as shown by the state diagram in Figure 3.5.

The acquisition and synchronization works in the following way: A slightly modified version of double dwell serial search scheme is used [13][15]. The system starts in the first dwell state (aq1). The PN code is fed into a shift register which is long enough to hold the whole PN sequence. A bank of correlators are tapped to the shift registers in such a way that the code phase of the incoming signal can be detected if there is a high correlated output from one of the correlators at the end of each correlation cycle. The clock signal to the shift register is designed such that the phase can be half-shifted once after a complete PN sequence is generated. Therefore, the local code is sliding half a chip every cycle, until one of the correlator outputs exceeds the predetermined threshold (hit $1=1$ ). The system then switches to the second dwell state (aq2) and correlates for another cycle. If again, the output of the same correlator exceeds the threshold (hit2 $=1$ ), the decode is said to be synchronized and the system advances to the tracking state (lock1). Otherwise, the system switches back to the first dwell state (aq1) and the clock shifts half a chip again after each PN cycle and the process is repeated.

In the tracking mode (lock1), a pair of correlators are employed to correlate the half-chip advanced and retarded versions of the received signal. The difference of the outputs from the 2 correlators is D/A converted and fed to a voltage controlled oscillator (VCO) that adjusts the clock phase continuously. Meanwhile, the threshold detector continues to monitor the outputs 


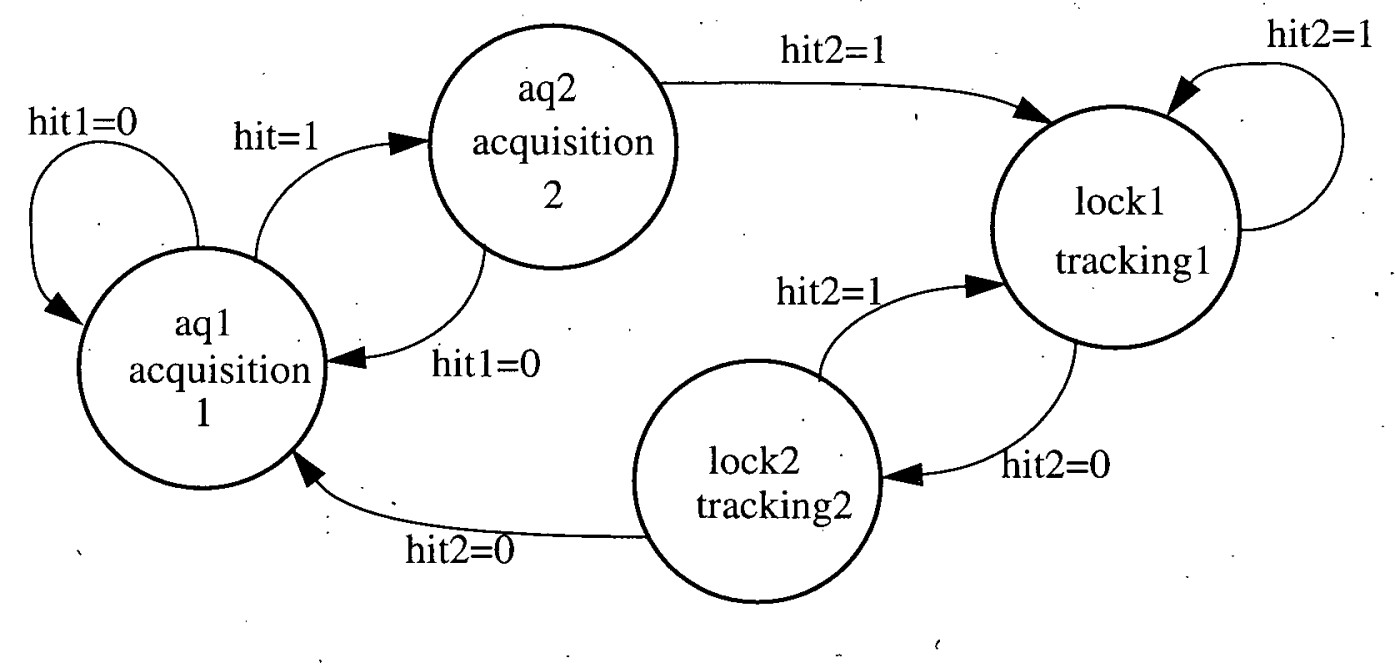

Figure 3.5 State.Diagram of the Double Dwell Search Lock Scheme

from the correlators. If none of them exceeds the threshold (hit $2=0$ ), the system will go to the second lock state (lock2) and continue to decode data. The system will go back to lock1 if the next correlation exceeds the threshold (hit $2=1$ ). Otherwise, the tracking is lost and the system switches back to the very first acquisition state (aq1). The reason for adding a state lock 2 is as follows: When the noise in the channel is high, there may be chances that none of the correlator outputs exceed the threshold while the system is still synchronized and decoding correct data.The the lock 2 state is therefore added for the system to declare the loss of synchronization when the correlator outputs are lower than the threshold twice in a row.

\subsubsection{The Decision Device}

The decision device compares the correlator (from the despreader) outputs so as to locate the correlator which gives the largest output. The "location" of the correlator provides the information for the data reassembler to look up the symbol corresponding to that amount of phase shift. The major component is a digital comparator. By using a bank of digital comparators 
connected to a bank of correlators, and with some simple logics, the correlator that gives the highest correlation can be identified.

\subsubsection{The Data Reassembler}

The data reassembler compares the "location" of the correlator which gives the largest correlated output with that of the correlator which correlates with the reference PN sequence. The difference of the location is the symbol value and hence the output of the decoder.

\subsubsection{The PC Interface}

The PC interface is responsible for reading the data from the CPSK decoder and storing into the PC's memory. Since the PC cannot receive the data in synchronization with the decoder, some first in first out (FIFO) memory buffer is inserted to accommodate the situation [18]. Also, the interface is able to read the status like the search-lock state of the decoder and give a reset on the CPSK decoder to restart the data acquisition process. All the data flow and signal control are controlled by a piece of software. The block diagram of the interface is shown is Figure 3.6.

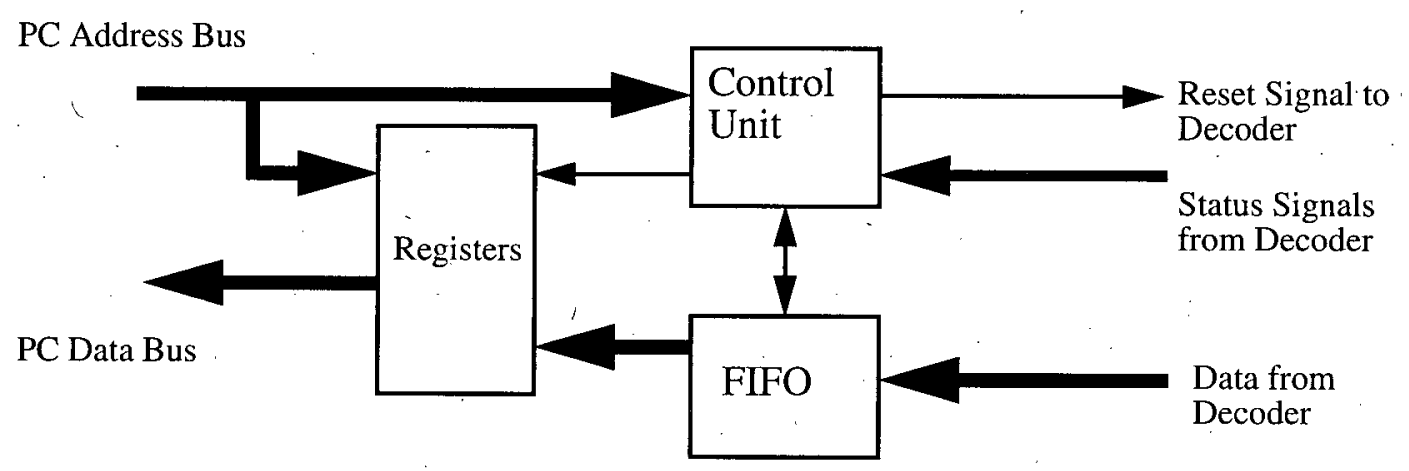

Figure 3.6 Block Diagram of the PC Interface 


\section{Chapter 4 The Receiver Implementation}

The details in implementing the IF demodulator, the CPSK decoder, the PC interface, and the control software are discussed in the following sections.

\subsection{The IF Demodulator}

The block diagram for the IF demodulator is shown in Figure 3.2. As the basic circuit is adapted from the WLAN receiver front end in [5], only a brief description on the basic circuit and the modifications made are presented here. The design details of the front end can be found in [12]. The major modification includes the addition of a Costas loop to provide a synchronized local oscillator (LO) frequency. with respect to the carrier frequency. In the IF demodulator, I and Q demodulation are performed by a low cost Mini-Circuits MIQY-140D demodulator (Figure 4.1).

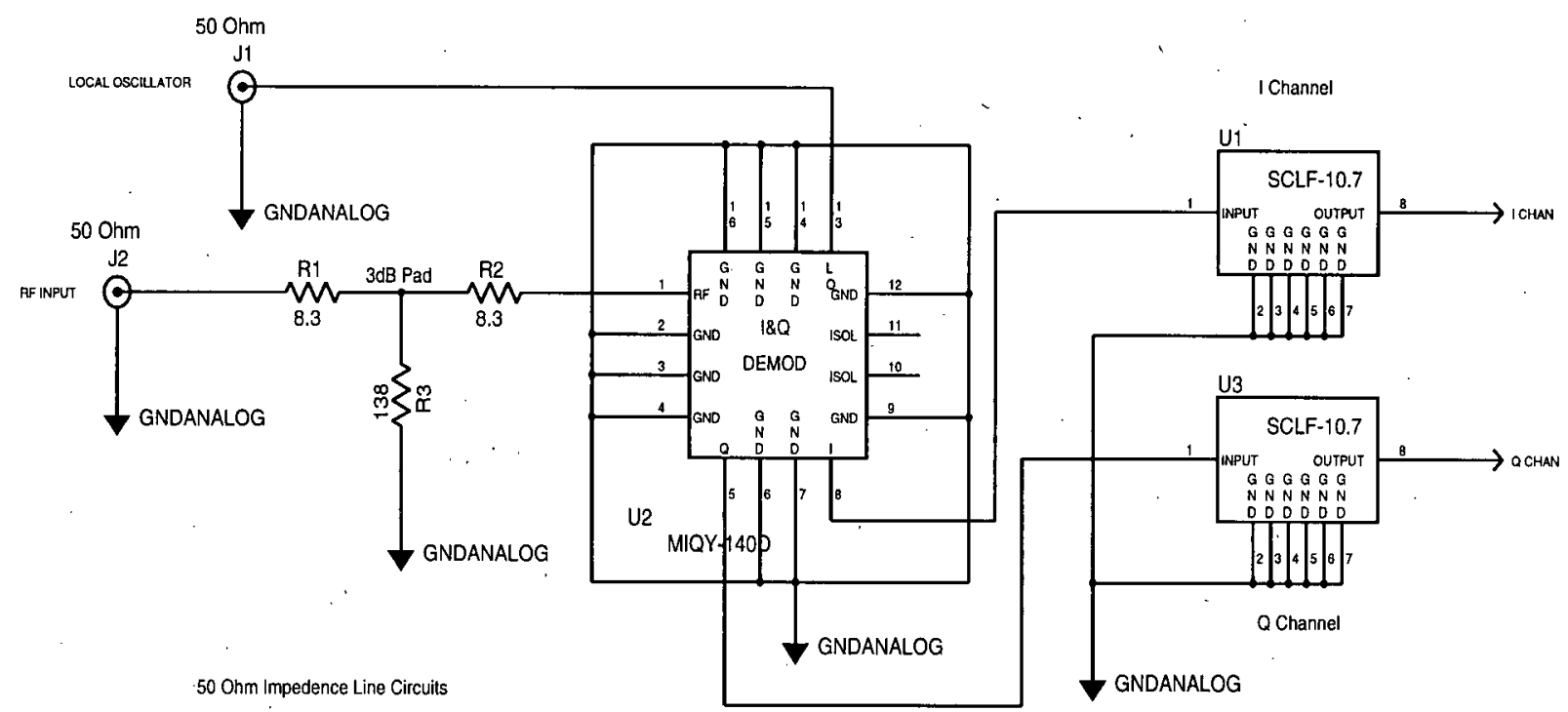

Figure 4.1 The BPSK Demodulation Section of the IF Demodulator

Although this demodulator provides good harmonic suppression, additional low pass 
filtering has been added at the demodulator I and Q outputs to improve harmonic suppression above $11 \mathrm{MHz}$ further. Low pass filtering at $11 \mathrm{MHz}, 1 \mathrm{~dB}$ cutoff, is provided by Mini-Circuits SCLF-10.7 filters. $11 \mathrm{MHz}$ is selected to allow for possible future increase in chipping rate goals so that a PN code chipping rate up to 4 Mcps can be used. The setting of the cutoff frequency can be found in [11]. I and Q channel signals are then amplified and level shifted to the range required by the $\mathrm{A} / \mathrm{D}$ converter which is between 0 and $2 \mathrm{~V}$. A second stage active first order low pass filter is combined with the gain stage to provide dominant pole cutoff at a calculated optimal frequency cutoff point to maximize signal to noise ratio (Figure 4.2). Please refer to [12] for the details in cutoff frequency calculation.
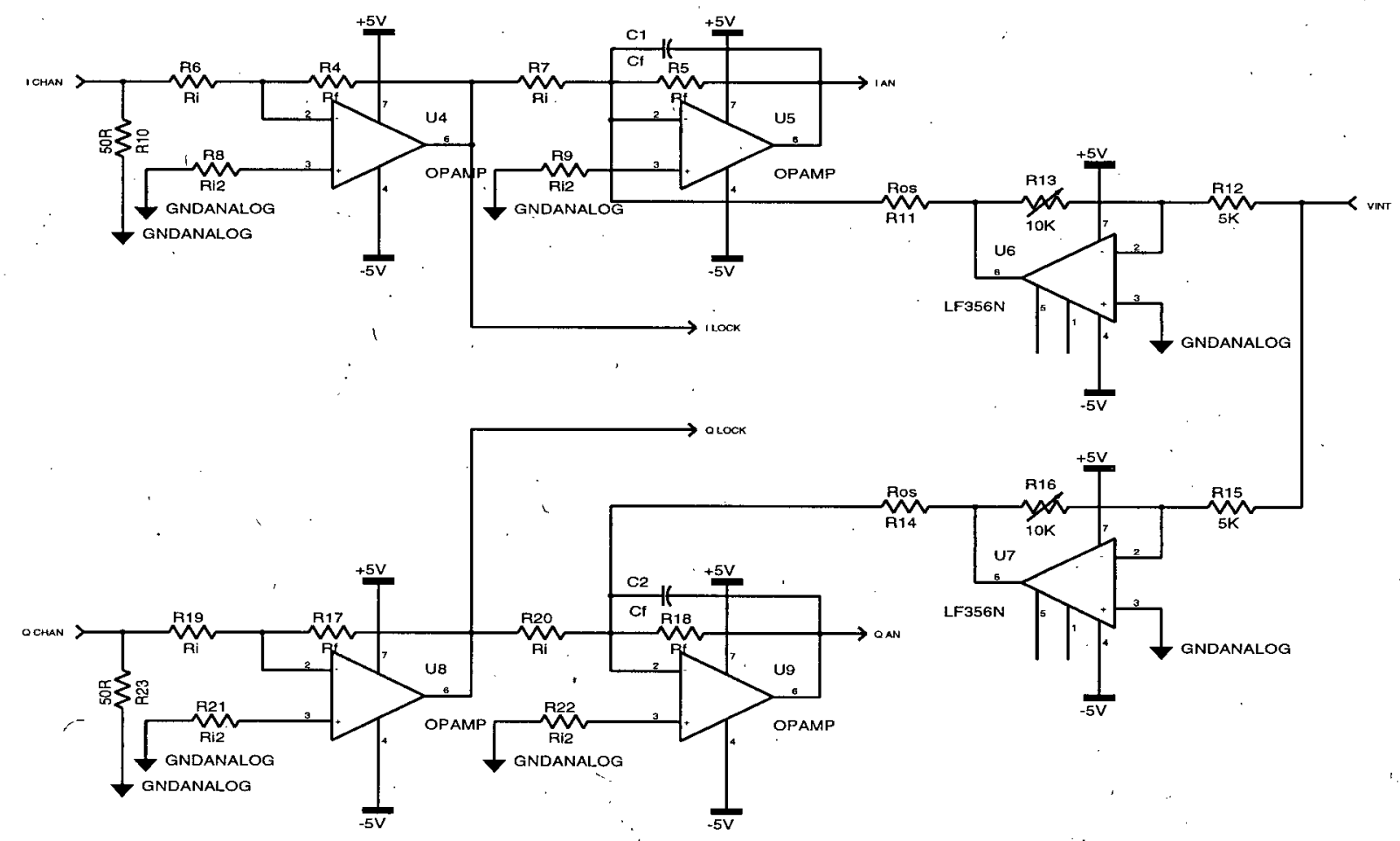

Figure 4.2 The Filter and Amplifier Section of the IF Demodulator

The feedback part of the Costas loop is formed by an EXAR 2208 four quadrant multiplier and a Mini-Circuits POS-150 VCO. As shown in Figure 4.3, the I and Q channel signals are fed 


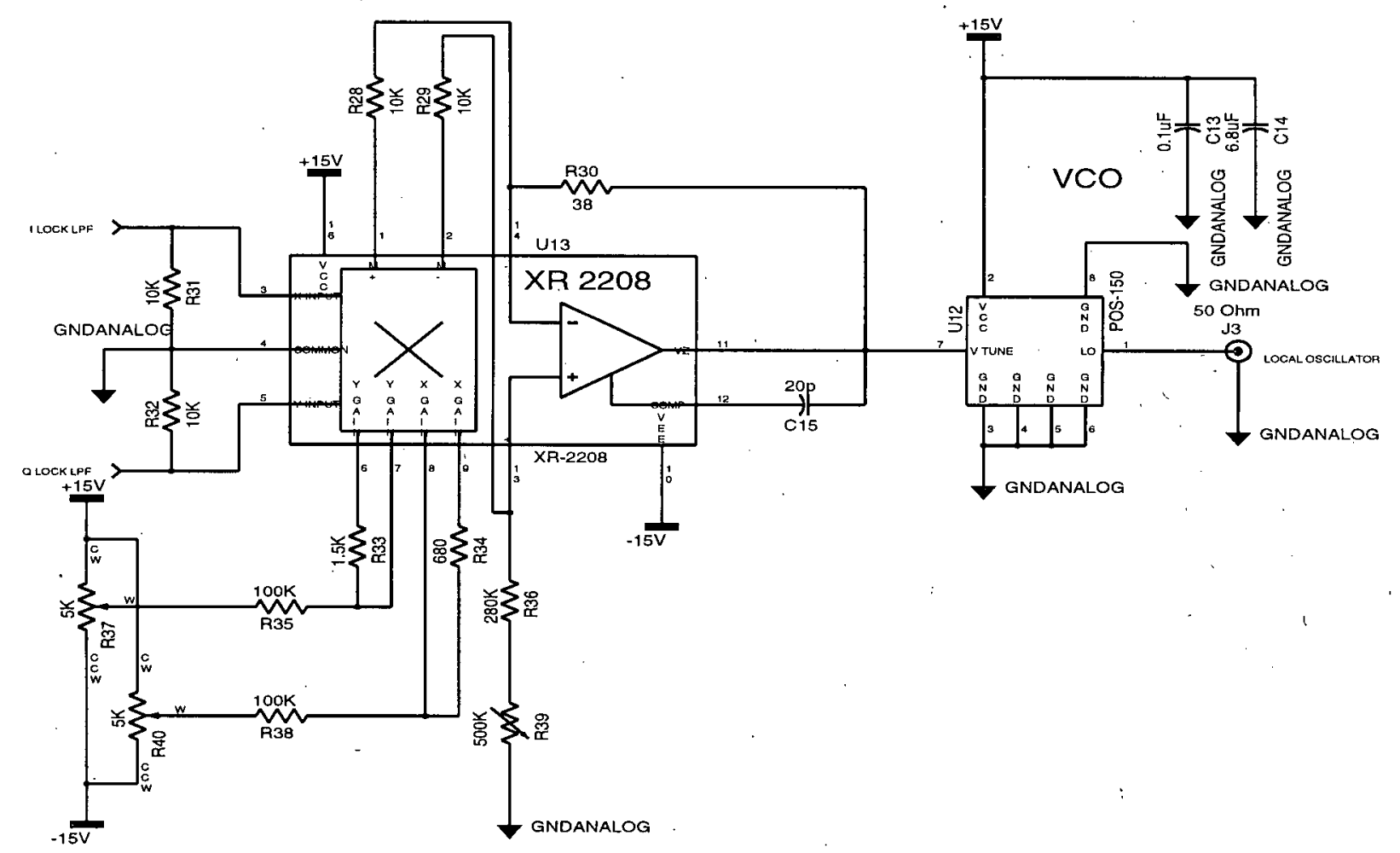

Figure 4.3 The Costas Loop Section of the IF Demodulator

into the multiplier. The analog multiplier output is gain adjusted and level shifted to provide a control signal to the $\mathrm{VCO}$ centered at $140 \mathrm{MHz}$ which in turn provides the LO signal required for demodulation. The demodulated signals are then band-pass filtered and fed into an Analog Device AD9058 JD dual 8-bit A/D converter (Figure 4.4). Note that the I and Q channels must carry the same baseband signal for the Costas loop to work properly. The CPSK decoder provides a sample signal at twice of the chipping rate to sample the demodulated signal and convert it into digital form for decoding. Only the A/D output from the I channel is used by the CPSK decoder. 


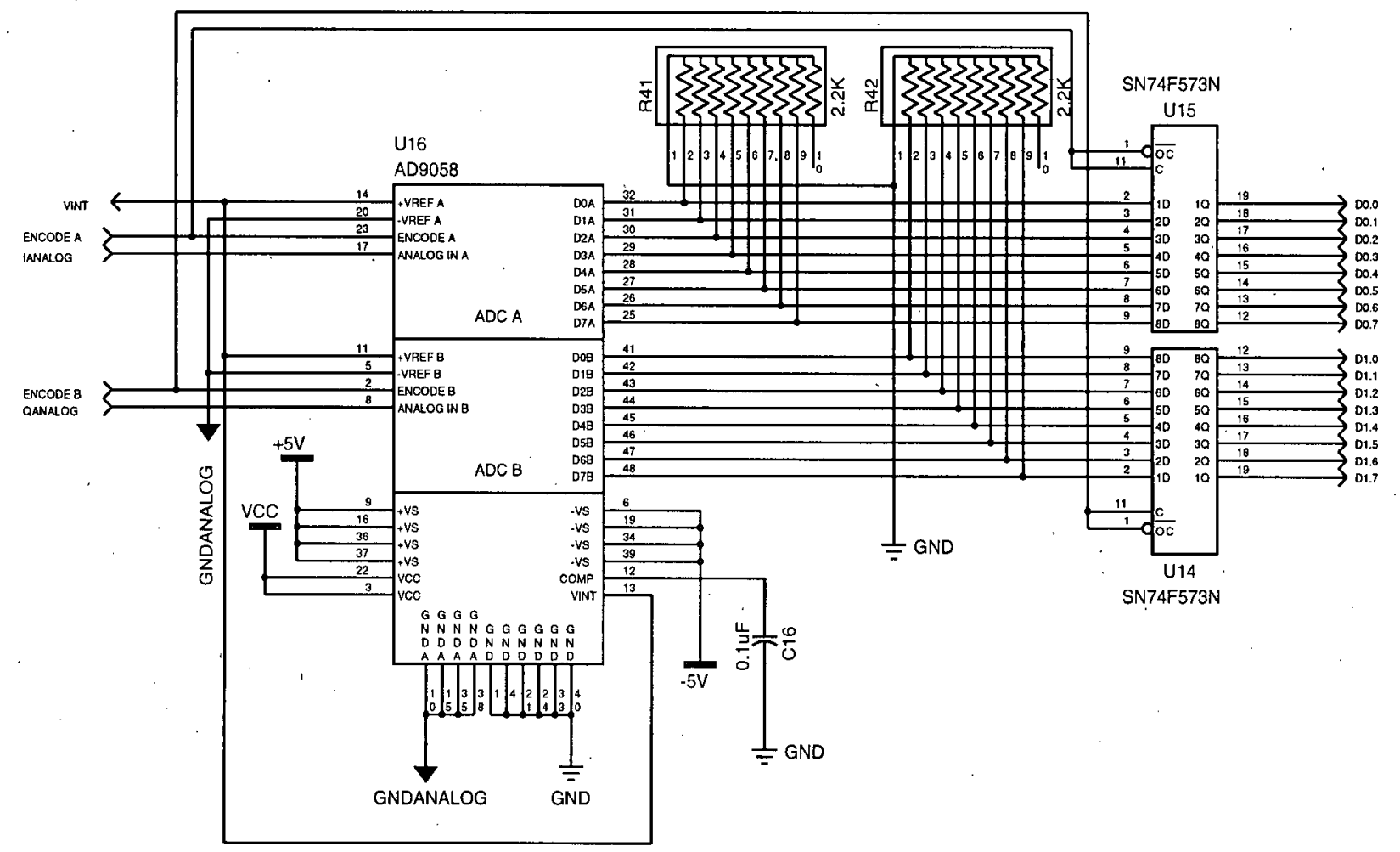

Figure 4.4 The A/D Converter Section of the IF Demodulator 


\subsection{The CPSK Decoder}

The CPSK decoder receives the digital data from the I channel A/D output of the IF demodulator and decodes the signal using CPSK signaling scheme. Although the decoding process is done digitally, the master clock needs to be adjusted by some analog circuits in order to maintain continuous tracking. Therefore, the CPSK decoder is composed of 2 sections, the digital and analog section. The digital section is implemented on a Xilinx 4010 FPGA device programmed using Cadence System [20] and the analog section is implemented with discrete components. The block diagram of the CPSK decoder is shown in Figure 4.5. Since the decoder is designed to decode CPSK signal with a PN sequence length of 127 and word (symbol) size of 3 as specified in Chapter 3, a 127-stage shift register and a PN code generator are used to provide PN codes with different phase shifts and 8 correlators are used to detect the 8 different values of a 3 bit word. The outputs of the correlators are then fed into 8 threshold devices and a decision device for changing the state of the system (i.e. the state of the search lock logic in Figure 3.5) according hit 1 and hit 2 in the figure, and locating the correlator which gives the largest correlated output. During acquisition mode; the PN sequence is shifted half a chip once every PN code cycle and this is done by the clock shift module. When the system is in tracking mode, a pair of early and late correlators and a 16-bit subtractor are used to determine the phase difference of the incoming signal and the local PN code. The difference value is then fed to the analog section for adjusting the master clock of the decoder [14]. The details of the design of each sub-module of the decoder are presented in the following subsections.

\subsubsection{Conventions Used in the Schematics}

In the following subsections, the diagrams are extracted from the schematics composed 


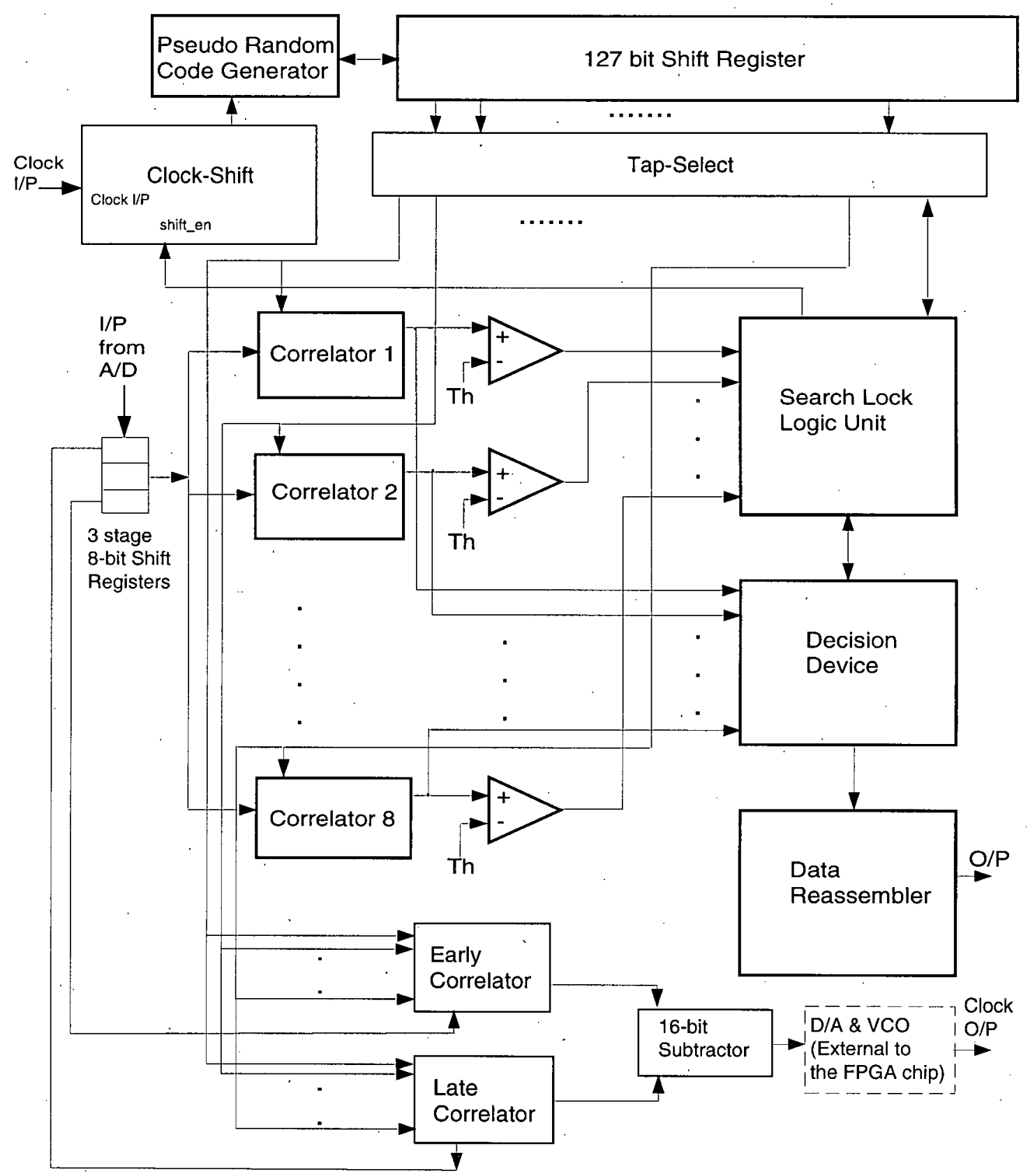

Figure 4.5 The Block Diagram of the CPSK Decoder

with Cadence system. Some of the important symbols and I/O conventions are addressed as shown by an example in Figure 4.6(a). The inputs $a<15: 0>$ and $b<15: 0>$ represent 216 bit bus signals being fed into the symbol named COMPMC16. The outputs of the symbol are gt and lt 
respectively. The description of the symbols used in this chapter are shown in Table 4.1. Moreover

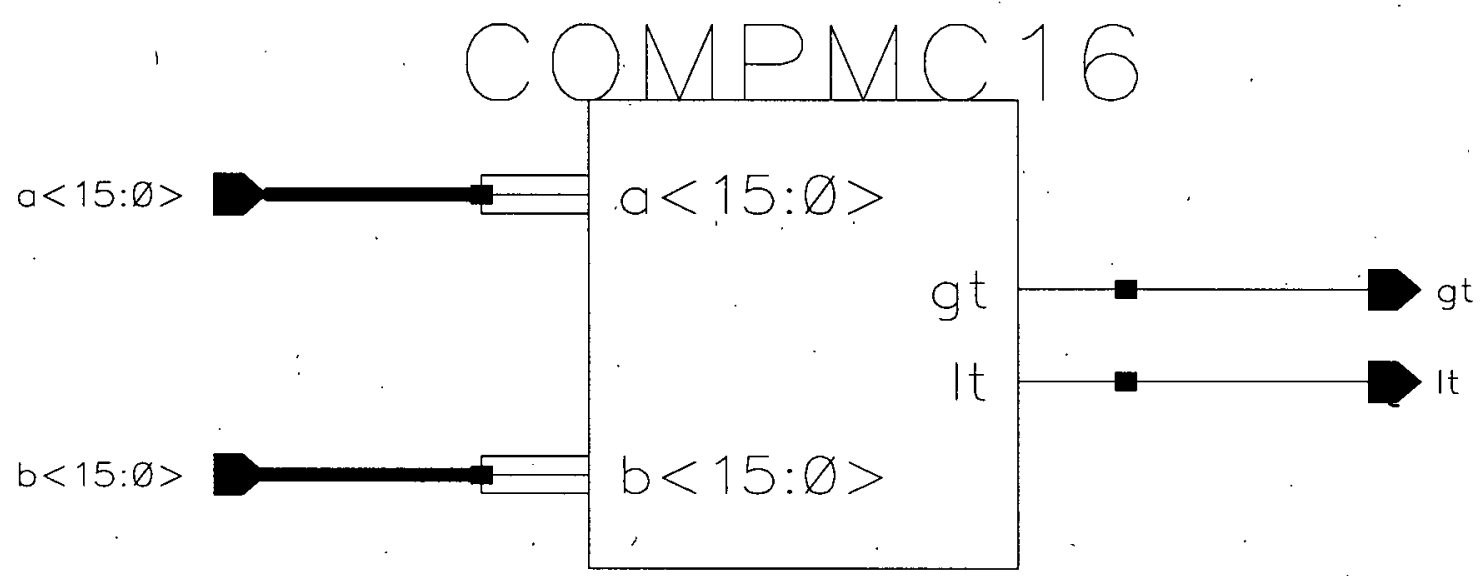

(a)
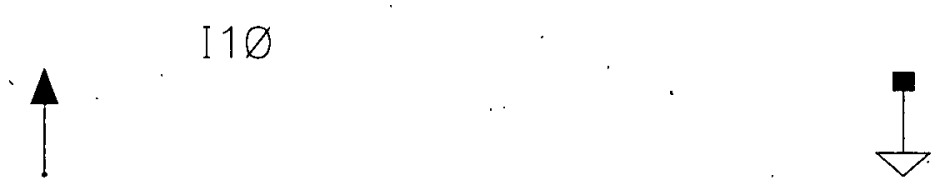

(b)

(c)

Figure 4.6 Symbols and I/O Conventions Used in the Schematics Composed with Cadence System

2 special symbols, as shown in Figure 4.6(b) and Figure 4.6(c), represent the connection to power and ground, respectively.

Table 4.1 Descriptions of the Symbols

\begin{tabular}{|l|l|}
\hline \multicolumn{1}{|c|}{ Symbol } & \multicolumn{1}{c|}{ Description } \\
\hline ACCx & x-bit accumulator \\
\hline ADDX & x-bit adder \\
\hline ANDxBy & x input AND gate with y bit inverted \\
\hline ADSUx & x-bit adder/subtractor \\
\hline
\end{tabular}


Table 4.1 Descriptions of the Symbols

\begin{tabular}{|l|l|}
\hline \multicolumn{1}{|c|}{ Symbol } & \multicolumn{1}{c|}{ Description } \\
\hline BUFEx & x-bit buffer with enable \\
\hline BUFTx & x-bit tri-state buffer \\
\hline CBxCE & x-bit binary counter \\
\hline COMPMCx & x-bit comparator \\
\hline FDC & D flip flop with asynchronous clear \\
\hline FDCE & D flip flop with asynchronous clear and enable \\
\hline FDR & D flip flop with synchronous reset \\
\hline FTC & T flip flop with synchronous reset \\
\hline INV & Inverter \\
\hline M2_1 & 2 to 1 multiplexer \\
\hline NORxBy & x input NOR gate with y bits inverted \\
\hline SR16CE & 16 bit shift register \\
\hline XOR2 & 2 input exclusive OR gate \\
\hline
\end{tabular}

\subsubsection{The Digital Correlator and the Threshold Device}

The incoming signal from the IF demodulator is correlated with different phase shifts of the local PN sequence by using digital correlators. The actual signal levels of the incoming signal and the local PN sequence are mapped to positive integers as shown in Table 4.2 and Table 4.3. where $\mathrm{M}$ is the peak level of the signal. 
Table 4.2 Digital Representations of Incoming Signal

\begin{tabular}{|l|l|}
\hline Real Value of incoming signal & Digital Representation (8 -bit number) \\
\hline -M to 0 & 0 to 127 \\
\hline 0 to $\mathrm{M}$ & 127 to 255 \\
\hline
\end{tabular}

Table 4.3 Digital Representations of PN Sequence

\begin{tabular}{|l|l|}
\hline Real Value of PN code & Digital Representation (1 bit number) \\
\hline+1 & 1 \\
\hline-1 & 0 \\
\hline
\end{tabular}

Since a PN sequence $c(i)$ consists of only +1 and -1 values, the digital correlation represented by

$$
R=\sum_{i=1}^{127} c(i) d(i)
$$

can be implemented by using an accumulator with increment and decrement capability: When c(i) is $+1, \mathrm{~d}(\mathrm{i})$ is added into the accumulator. When $\mathrm{c}(\mathrm{i})$ is negative, the value of the accumulator is decreased by d(i). To determine the size of the accumulator, the maximum and minimum possible correlation values need to be calculated. The correlation is a maximum when code phase of the incoming signal is the same as that of the local PN code and the correlation is a minimum when the code phase of the incoming signal is the same as that of the inverted local PN code. A 127 chip PN sequence contains 64 1's and 63 0's. The maximum and minimum possible values are:

$$
\begin{aligned}
& R(\max )=64 \times 255 \times(+1)+63 \times 0 \times(-1)=16193=3 \mathrm{~F} 41_{H} \\
& R(\min )=64 \times 0 \times(+1)+63 \times 255 \times(-1)=-16065=-3 \mathrm{EC} 1_{H}
\end{aligned}
$$

To eliminate the handling of negative numbers, the accumulator is offset for 16065 or 
$3 \mathrm{ECl}_{\mathrm{H}}$ at the beginning of each correlation cycle and this offset is compensated by the adjustment of the threshold. Therefore the maximum value of the accumulator with offset is $3 \mathrm{ECl}_{\mathrm{H}}+3 \mathrm{~F} 41_{\mathrm{H}}$ $=7 \mathrm{E} 02_{\mathrm{H}}<\mathrm{FFFF}_{\mathrm{H}}$ and a 16-bit accumulator is employed to perform the required correlation. The circuit of the correlator is shown in Figure 4.7. The meanings of the I/O pins are described in

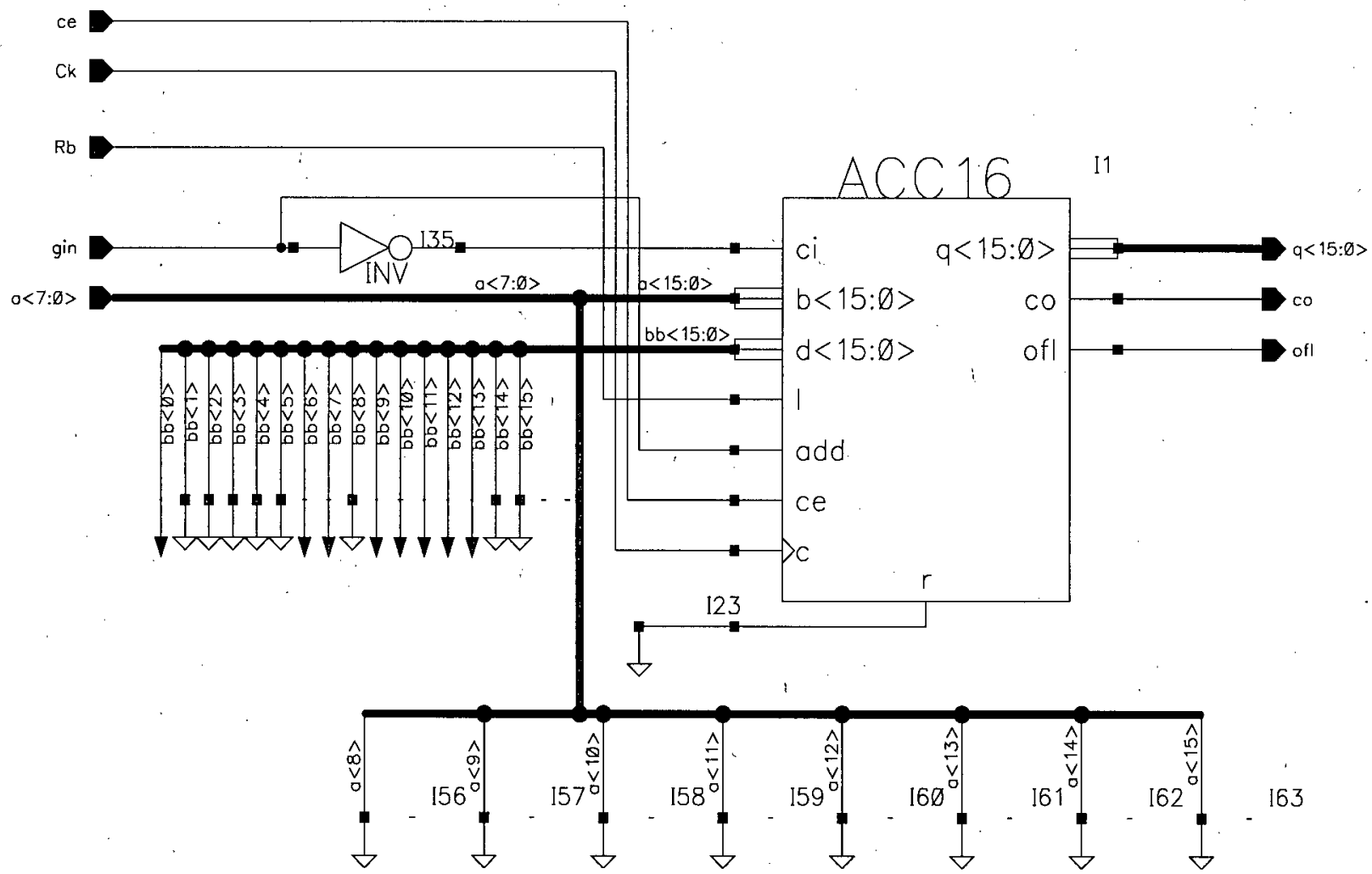

Figure 4.7 The Digital Correlator

Table 4.4.

The threshold device is used to determine whether the correlated value is large enough to declare a change of state from acquisition to tracking. As shown in Figure 4.8, the device is merely a 16-bit digital comparator that compares the correlated value with some threshold values. It is known from the.design of the IF demodulator that the signal feeding into the decoder has a 
180 degree of phase uncertainty. To combat this uncertainty, 2 thresholds thl and thu are selected in turn by the "compare" signal from the clock shift module to compare with the correlated output. The correlations of the in-phase and $180^{\circ}$ out-of-phase incoming signal with local PN Table 4.4 I/O Pin Definitions of the Digital Correlator

\begin{tabular}{|l|l|}
\hline \multicolumn{1}{|c|}{ Pin } & \multicolumn{1}{c|}{ Description } \\
\hline ce & enable input \\
\hline $\mathrm{Ck}$ & clock input \\
\hline $\mathrm{Rb}$ & reset \\
\hline gin & local PN code input \\
\hline $\mathrm{a}<7: 0>$ & 8 bit input signal \\
\hline $\mathrm{q}<15: 0>$ & 16 bit output signal \\
\hline co & carry out \\
\hline ofl & output overflow \\
\hline
\end{tabular}

code are shown in Figure 4.9. If the correlated output is greater than thu, the incoming signal is in phase with the local PN code. If the output is smaller than thl, the incoming signal is $180^{\circ}$ out of phase with the local PN code.

The input s (connected to "compare" in the clock shift module) is used to select whether thl or thr is used as the threshold for comparison. When the correlated value is lower than thl or higher than thu, the output o of the device becomes 1 . This output is used by the search lock logic to determine whether a system state change is needed. The change of state is discussed in detail in Section 4.2.7. The thresholds were originally chosen to be the midpoints of the triangular curves in Figure 4.9 with values thl $=201 \mathrm{~F}_{\mathrm{H}}$ and thu $=5 \mathrm{E} 22_{\mathrm{H}}$ respectively. However in actual implementation, thl is set to a higher value while thu is set to a lower value because the signal strength is usually not in full scale and the probability of losing lock is decreased. For example, when the 


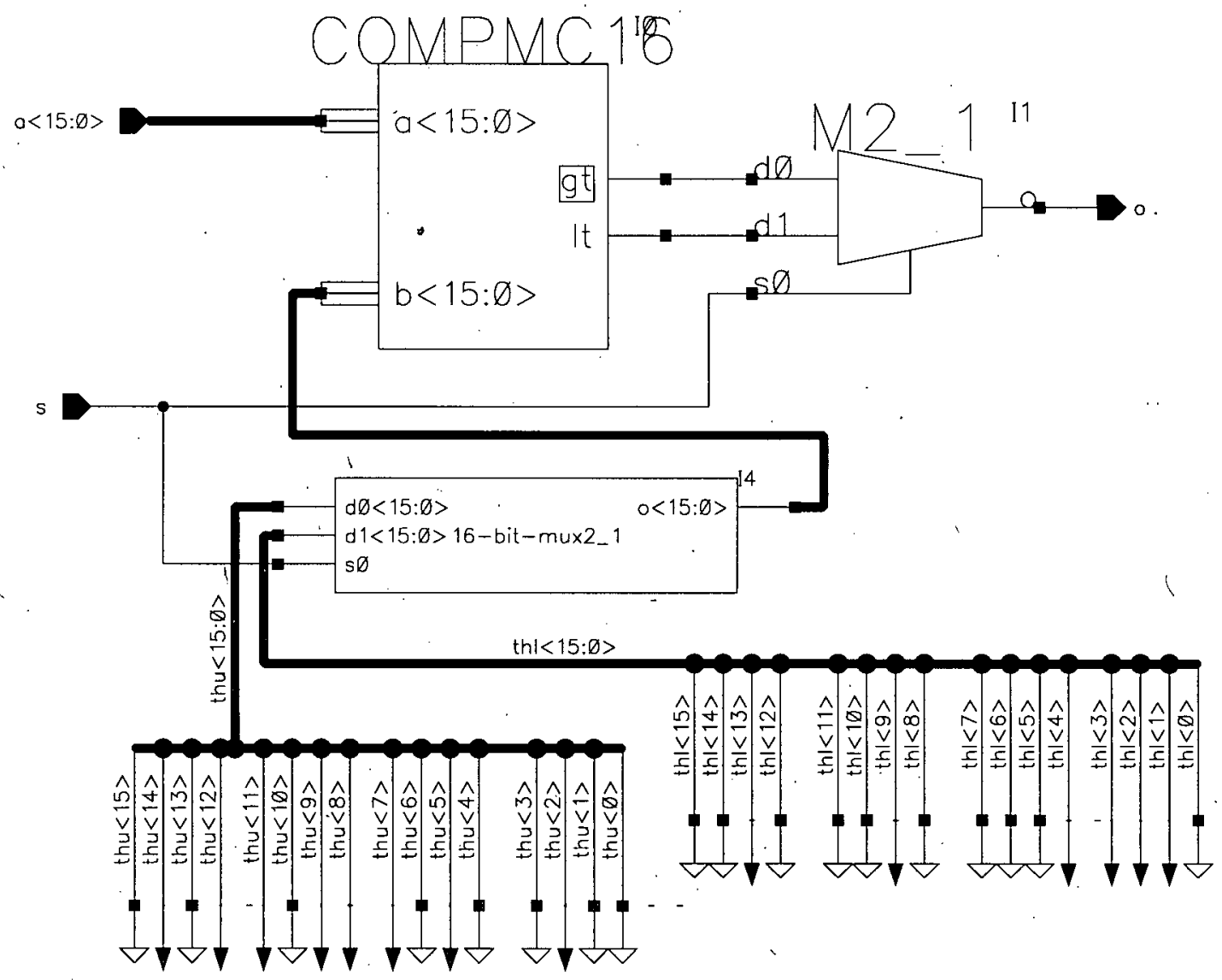

Figure 4.8 The Threshold Device

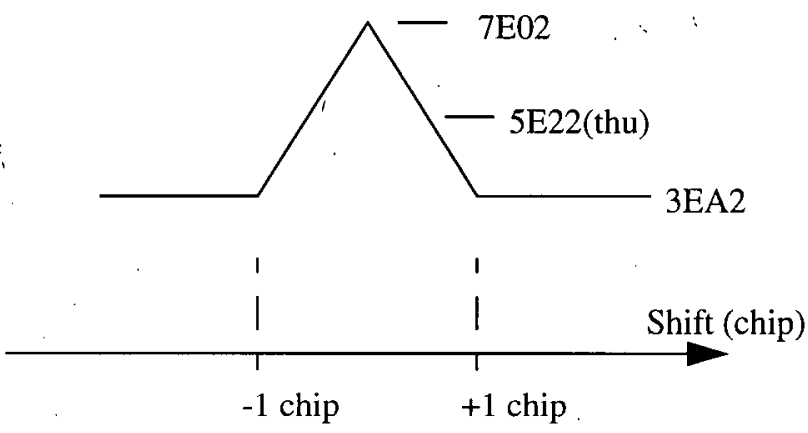

(a) With in-phase incoming signal

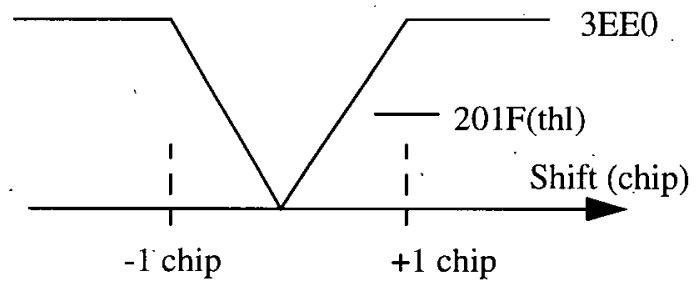

(b) With 180 degree out-of-phase incoming signal

Figure 4.9 Correlations of Incoming Signal with Local PN Code

amplitude of the input signal is $90 \%$ of the full scale value, the mid-point or the threshold should 
be adjusted to $90 \%$ of the ideal value. If the amplitude of the input signal (without any noise or jamming) is adjusted to the full scale value of the A/D converter, any extra noise or jamming signal will make the overall signal larger than the full scale value and the peaks of the digitized signal will be clipped or flattened and so the correlation contributed by the signal itself will be lowered.

\subsubsection{The PN Code Generator with Shift Register}

To generate a 127 chip PN sequence, a 7 stage linear feedback shift register is used. A linear feedback shift register consists of a delay line with taps that are modulo 2 summed and fedback to the first stage of the shift register. From [1], 7 different configurations of a 127 chip PN code generator are available. The one used in the CPSK decoder is one of the simplest type in that only the first and the last taps of the delay line are fed back to the first stage. To minimize the delay of the modulo 2 summers, a modular type generator is designed as shown in Figure 4.10 [1]. By feeding the clock signal to the input ck, the PN code is generated at the output q. Rs is the reset input to set q to zero when the decoder is reset.

In order to get different phase shifted versions of the local PN code, a 127 stage shift register is connected to the PN code generator to store the whole PN sequence and the taps to different flip-flop outputs of the shift register provide the required phase shifted PN codes.

\subsubsection{The Tap Select Module}

When the CPSK decoder is in the acquisition state (state aq1 in Figure 3.5), the incoming signal is fed into each of the 8 correlators to despread the signal with 8 different phase shifted versions of the local PN code that are tapped from the 127 stage shift register. The need of the tap 


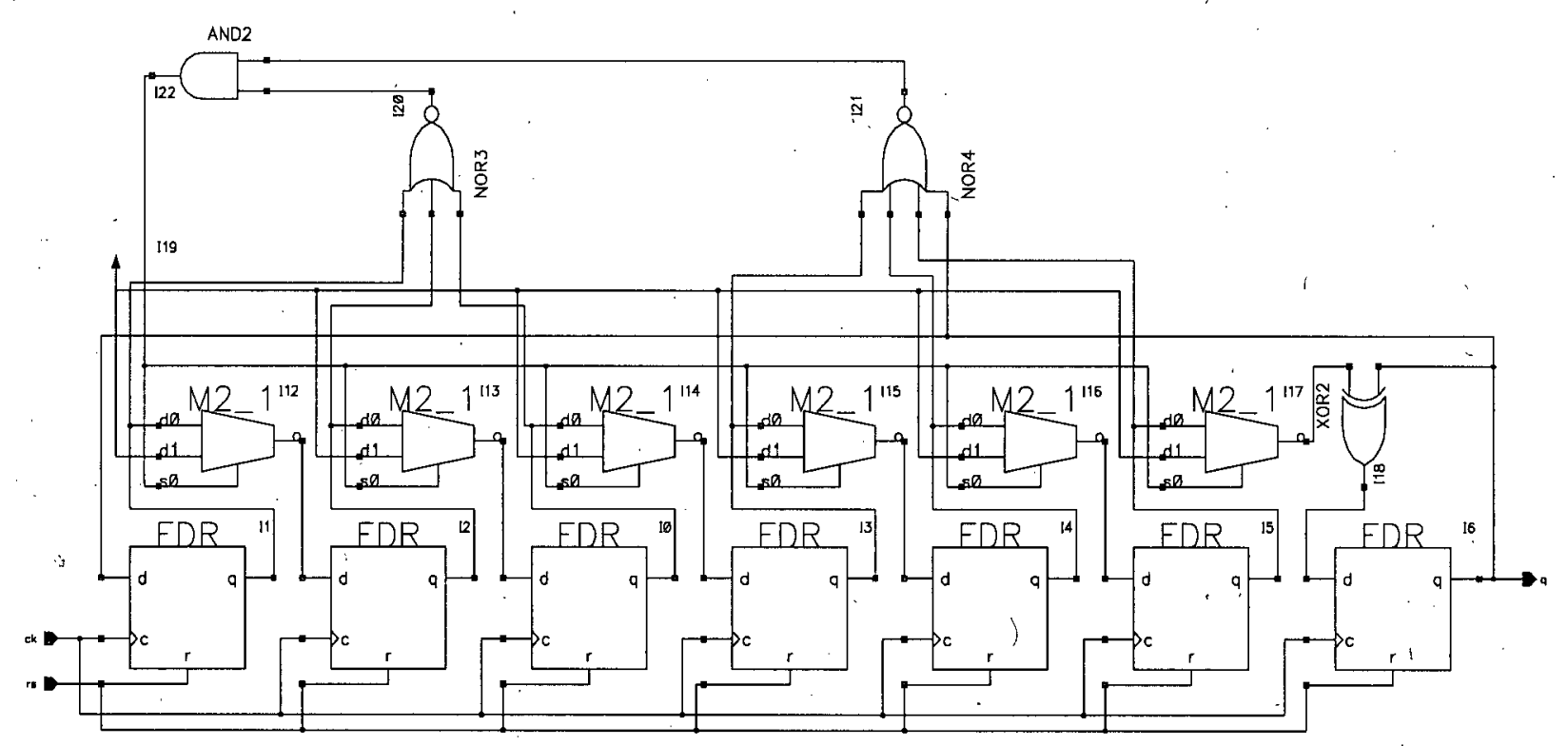

Figure 4.10 The 127 Chip PN Code Generator

select module is due to the fact that the phase shift between the first and the last sequence is 15 chips while the other adjacent pair of sequences are separated by 16 chips. During the acquisition mode, the phase shift between the PN codes fed into any adjacent pair of the correlators is 16 chips. When the system is first changed to the tracking mode, the address or the location of the correlator which gives the largest correlation is stored as the reference. When the reference correlator is not correlator 1 , the taps from the 127 bits shift register needs to be adjusted to make sure that the PN codes fed into the reference correlator and the one placed before it are separated by 15 chip while the codes fed to any other 2 adjacent correlators are differed by 16 chips.

As shown in Figure 4.11, the outputs $\mathrm{m} 0$ to $\mathrm{m} 6$ of the tap select circuit are used to select the taps from the shift register. The inputs ad<2:0> are connected to the register that store the reference address of the reference correlator. Since the phase difference between 2 adjacent PN codes is either 15 or 16 chips, 2:1 multiplexers controlled by $\mathrm{m} 0$ to $\mathrm{m} 6$ are connected to the shift 


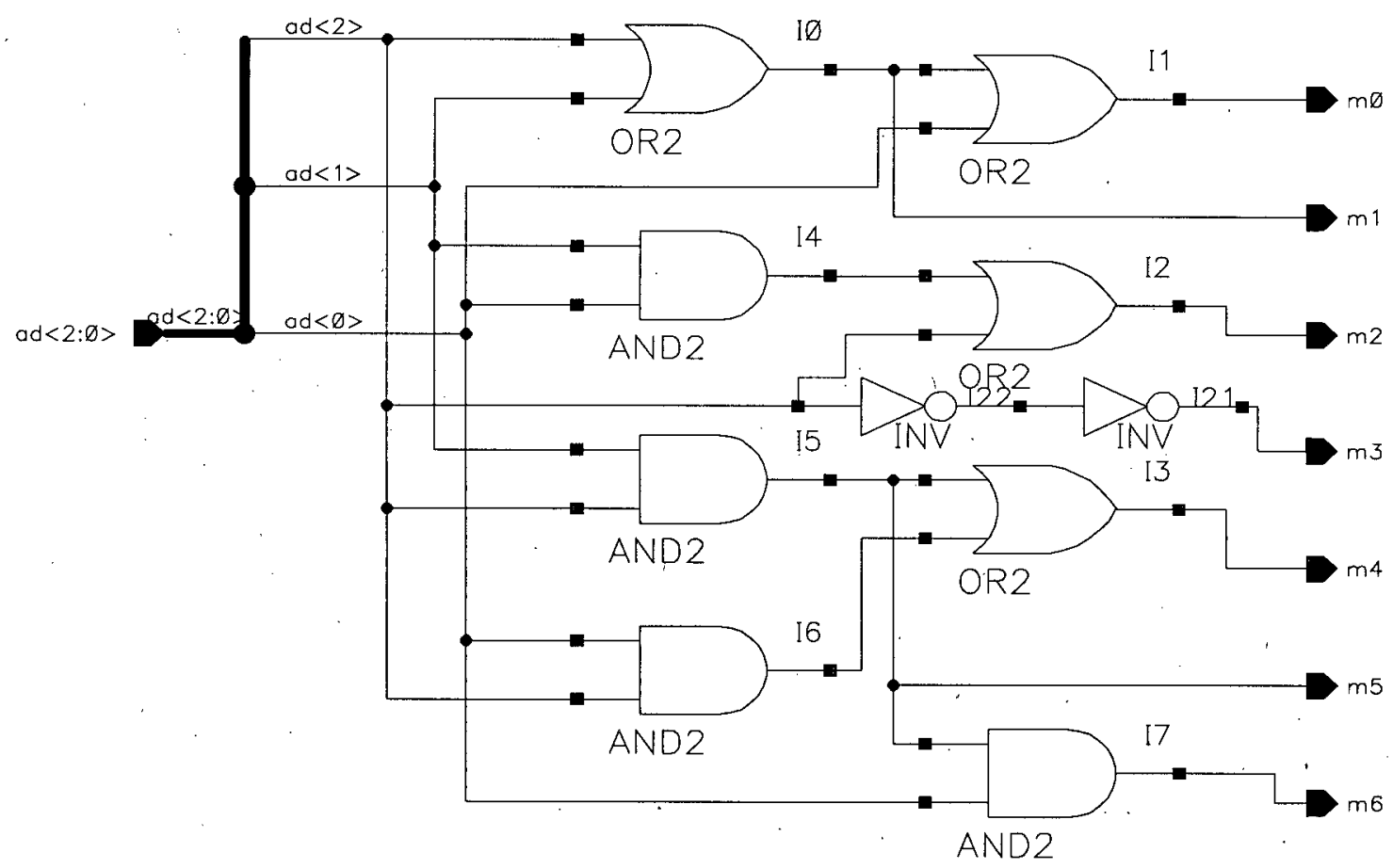

Figure 4.11 The Tap Select Module

register to select the correct phase shifted codes. The connections of the tap select module, the multiplexers, and the shift register are shown in the full schematic of the decoder in Figure 4.20.

\subsubsection{The Decision Device}

The decision device locates the correlator that gives the highest correlation. As shown in Figure 4.12, the 16 bit outputs $c 0<15: 0>$ to $c 7<15: 0>$ from the correlators are compared pairwise, and the results are fed into the logic circuit on the right hand side of the diagram for decoding the location or the address of the correlator $(\mathrm{psft}<2: 0>)$ with the highest correlation. It is known from previous sub-sections that the incoming signal has a 180 degree phase uncertainty. This uncertainty is resolved by the threshold devices as described in previous sections. The input u_l set by the threshold devices is used to select whether the decision is based on the largest value or the smallest value. Note that the comparators are 8 bits in size while $c 0<15: 0>$ to $c 7<15: 0>$ are 
16 bits in size. 16-bit comparators are not used mainly because they occupy too many configurable logic blocks (CLBs) in the FPGA chip (see Chapter 6 for a detailed description on CLBs). The total required resource of the whole CPSK decoder circuit will be too big to fit into the Xilinx 4010 chip if 16-bit comparators are used. Since the lower 8 bits of the correlator outputs contribute only $1 / 125$ of the full scale output value, the effect of these bits do not significantly affect the decision making process by the decision device so the lower 8 bits of the correlator outputs can be ignored [21]. An alternative to this approach is scaling down everything in the decoder so that the resolution of the input signal is reduced to 4 bit. However, this involves the redesign of other modules in the decoder. The replacement of the 16-bit comparators with 8-bit comparators is the easieśt way to accommodate the resource limitation of the chip.

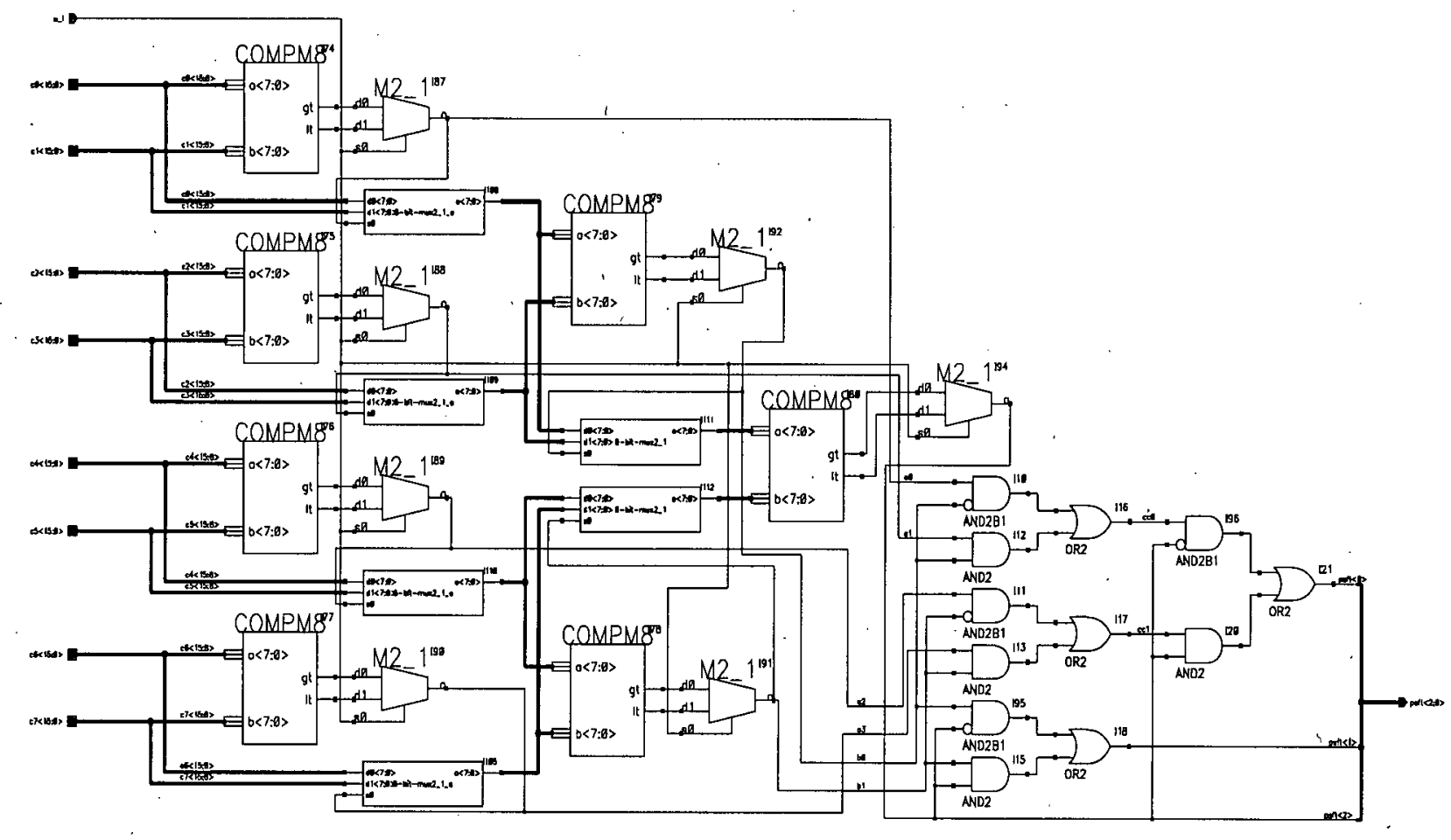

Figure 4.12 The Decision Device 


\subsubsection{The Clock Shift Module}

During the acquisition state (aq1), the local PN code needs to advance or retard half a chip once every correlation cycle (127 chips of correlation) in order to slide the phase of the local PN code until it is matched with the phase of the incoming signal. This is done by shifting the phase of the clock signal for the PN generator by half a chip using the clock shift module. This module also provides timing signals for doing data comparison, storage, state change, and data clear in the decoder.

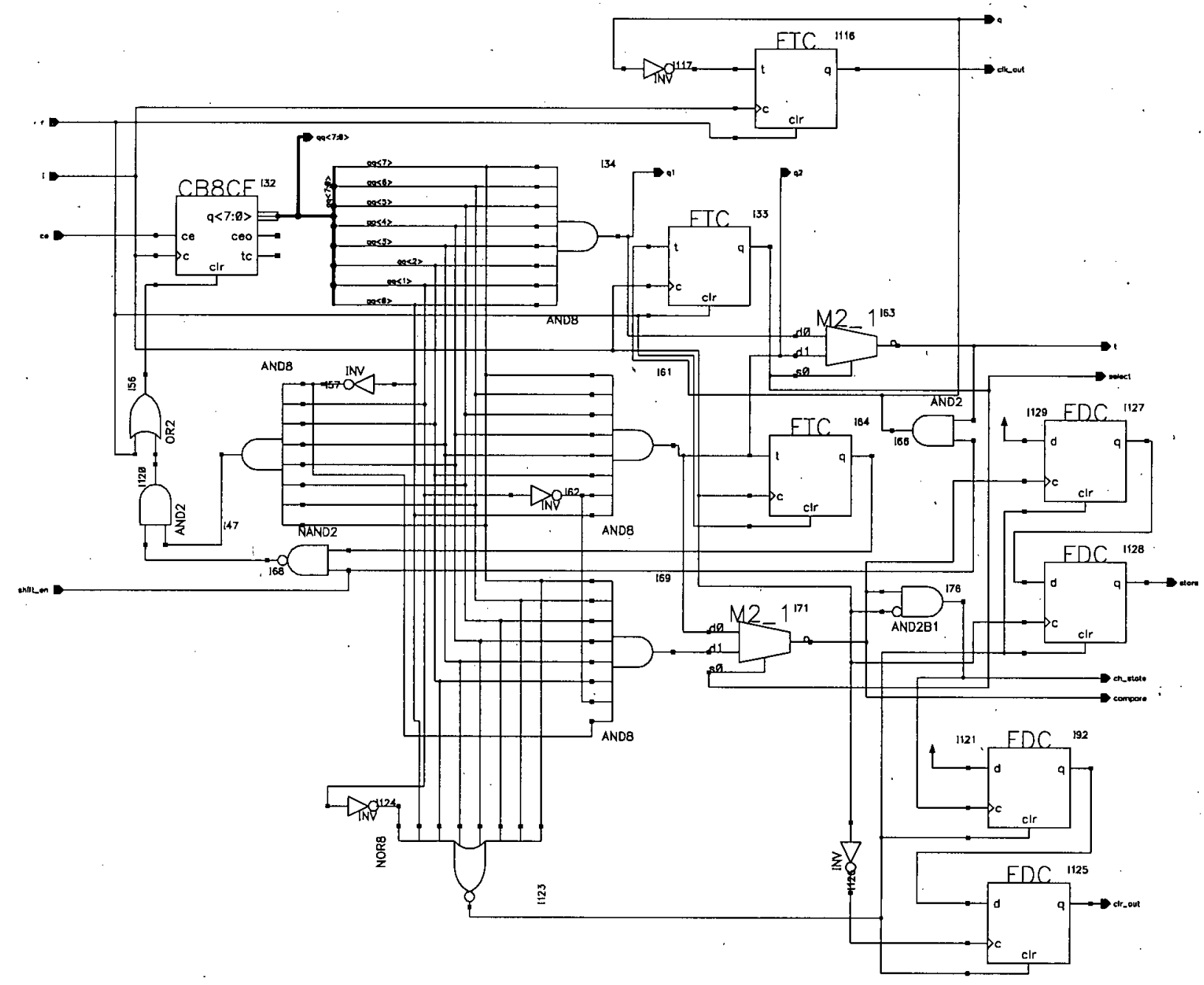

Figure 4.13 The Clock Shift Circuit 
To achieve the half-chip shifting, a clock signal with rate equal to twice the required chip rate is fed to the clock shift circuit and an 8-bit counter is added to keep track on each correlation cycle. The pin shift_en is used to select whether the chip is half-shifted. The resulting clock signal with half-chip shifting capability is the output clk_out as shown in the figure.

At the end of each correlation cycle, several tasks must be done sequentially before the data can be decoded correctly. The signals used to direct the tasks include compare, ch_state, store, and clr_out as shown in Figure 4.13. Compare is used to switch the thresholds for detecting out-of-phase incoming signal. Ch-state is used to change the state of the search lock logic according to hit 1 and hit2. Hit 1 is set when any of the threshold device output is 1 indicating that a high correlation is detected. Hit2 is set under one of the two conditions: 1) When the search lock state is in aq1, hit $1=1$, and the address of the reference correlator is equal to the address of the correlator which gives the highest correlation output at the end of the present correlation cycle. 2) When the search lock state is in either lock 1 or lock 2 and hit $1=1$. Store is used to store the reference address or location of the correlator that gives the highest correlation during acquisition mode and store the decoded data during the tracking or lock mode. Finally, the values stored in the correlators are cleared by the cli_out signal before the start of another correlation cycle. These task are completed in the last 2 chips (or 4 clock cycles) of each correlation cycle. Since the last 2 chips of each correlation cycle are not correlated, each correlated value is roughly decreased by $2 / 127$ or $1.6 \%$ and this can be compensated by lowering upper threshold thu and raising the lower threshold thl by the same amount. Three outputs namely $\mathrm{q}, \mathrm{t}$, and select are used for testing purpose and are not connected externally. 


\subsubsection{The Search Lock Logic Module}

The search lock logic is implemented using a modified double dwell serial search scheme [15] as shown in Figure 3.5 and the schematic is shown in Figure 4.14. Aq1, aq2, lock1, and lock2 are 4 possible states of the decoder. These states are represented by a 2-bit binary number \{qa qb where qa is the MSB of the number. The mapping of aq1, aq2, lock1, and lock2 to $\{\mathrm{qa}$ qb $\}$ is shown in Table 4.5. Note that lock 1 and lock 2 cannot be distinguished by \{qa qb\}. The 2 lock states are the internal states of the search lock logic and only the or-ed value of the 2 states is significant to the other parts of the decoder. The change of state is governed by 2 signals, hit 1 and hit 2 as represented by $\mathrm{h} 1$ and $\mathrm{h} 2$ in the schematic respectively. Pin ck is connected to the ch_state output of the clock shift circuit to update the search lock state. The purpose of $\{q a \mathrm{qb}\}$ are used externally as follows. When $\{\mathrm{qa} \mathrm{qb}\}=\{10\}$, the address of the reference correlator is stored; When $\{q \mathrm{qab}\}=\{11\}$, the decoded output is stored in the output register. the tracking loop is enabled, and the PC interface is signalled (along with clr_out in clock shift module) to read the decoded output.

Table 4.5 Representation of the Search Lock States

\begin{tabular}{|l|c|}
\hline \multicolumn{1}{|c|}{ Search Lock State } & qa qb \\
\hline System Reset & 00 \\
\hline aq1 & 01 \\
\hline aq2 & 10 \\
\hline lock1 or lock2 & 11 \\
\hline
\end{tabular}

\subsubsection{The Tracking Loop}

When the system first changes to the lock state (or tracking mode), the phase difference between the reference local PN code and that of the incoming signal may still be as large as $1 / 2$ chip. Also, both chip rates are not synchronized resulted in drifting of chips. A tracking loop is 


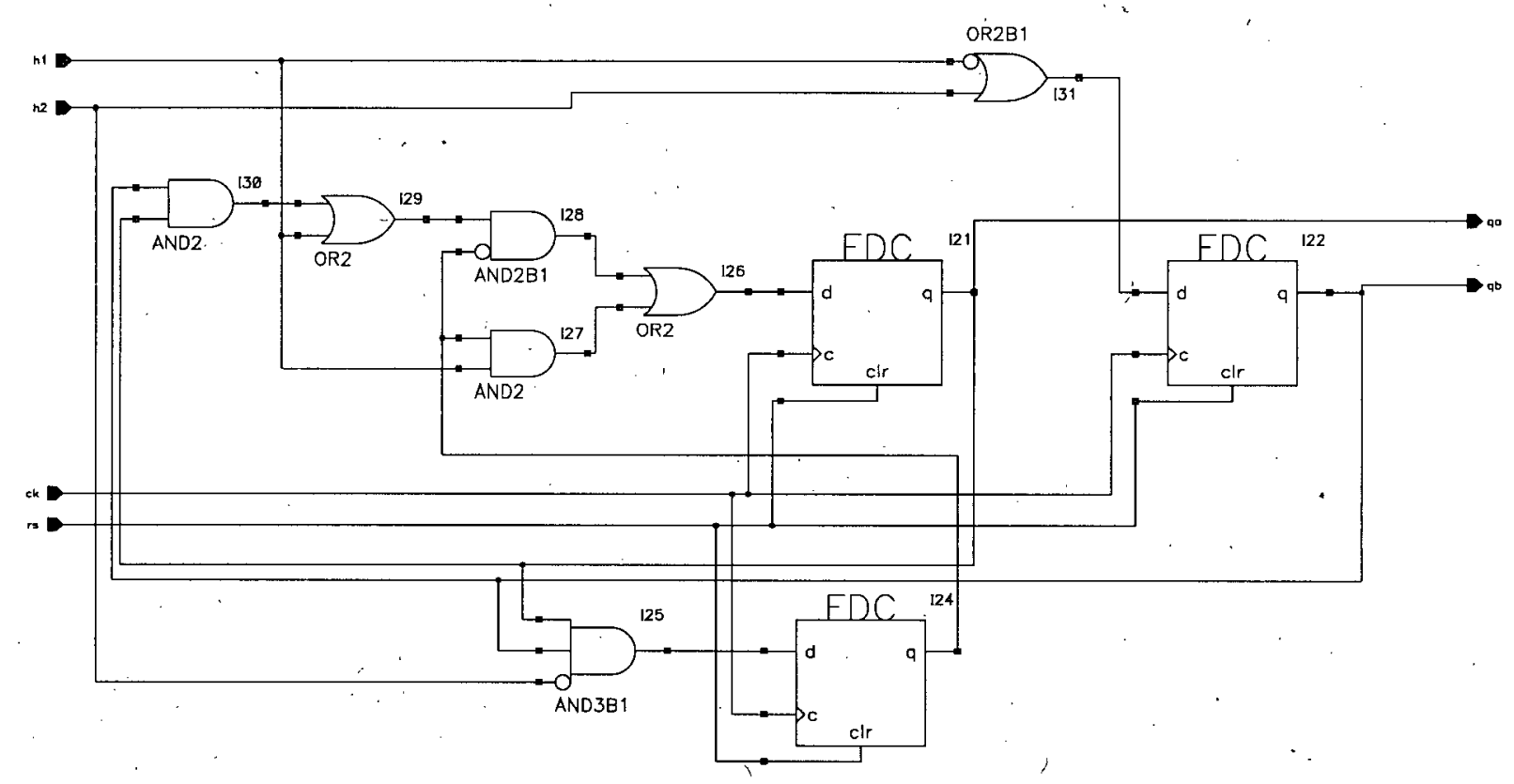

Figure 4.14 The Schematic of the Search Lock Logic Circuit

necessary to adjust the system clock to align the reference PN code phase with that of the incoming signal [13][16][17].

As shown in Figure 4.5, a pair of early and late correlators are used to calculate the correlation of the half-chip advanced and retarded versions of the incoming signal with the local PN code. The 2 versions of the incoming signal are obtained as follows. The incoming signal is sampled with twice the chip rate of the PN code and the samples are fed into an 8-bit-3-stage shift register (see Table 4.6 for the I/O pins definitions) as shown in Figure 4.15. Then, the late, punctual, and early versions of the incoming signal are obtained by the outputs from the first, second, and last stage of the shift register respectively. The outputs from the pair of correlators are then subtracted, D/A converted, and fed into a VCO for adjusting the master clock.

Since each symbol is decoded at the end of every correlation cycle, it is impossible to 


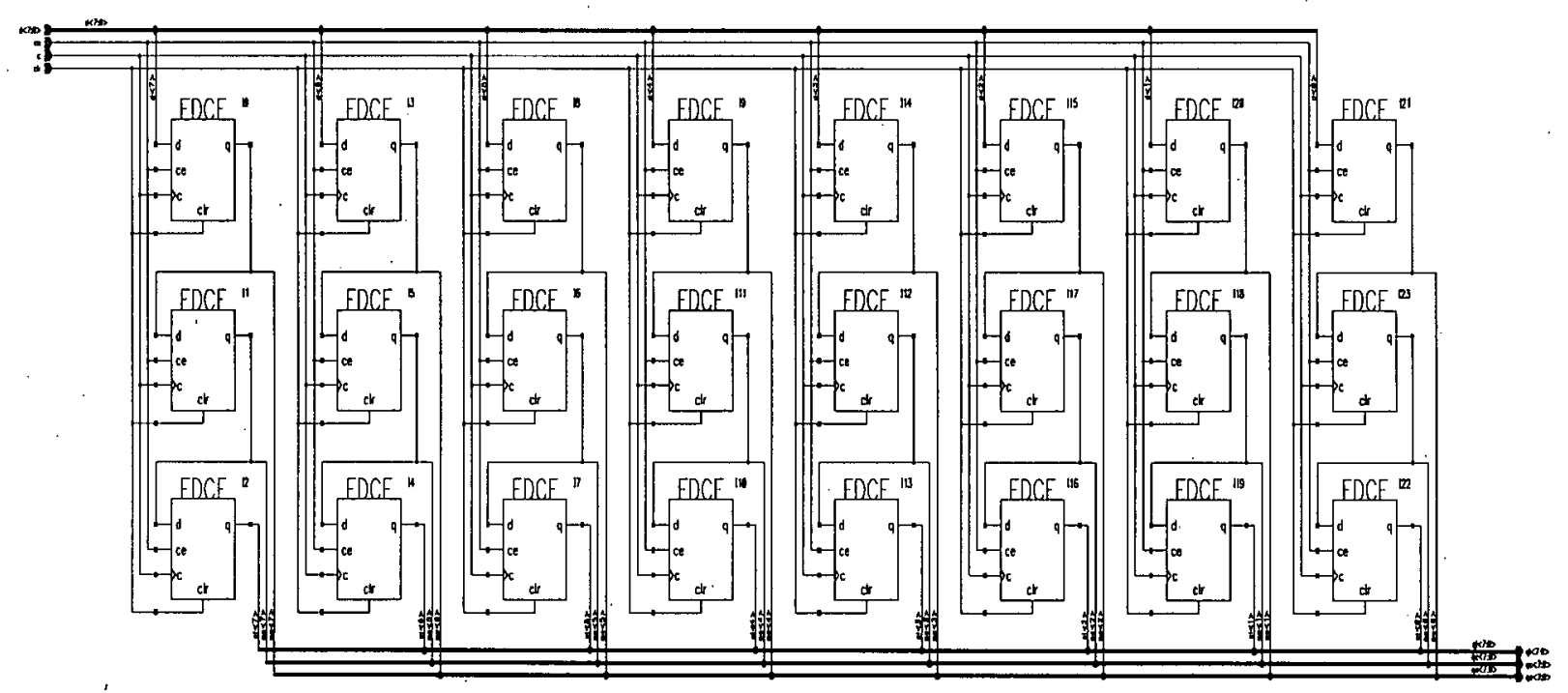

Figure 4.15 The 8-bit-3-stage Shift Register

choose which of the 8 versions of the PN code at the beginning of each correlation cycle to correlate with the early and late versions of the incoming signal for evaluating the correct phase error. One way to get the correct phase error is to store the incoming signal for a length of a whole PN sequence cycle. The approach would need 2 X 127 X $8=2032$ flip flops and this amount is much larger than the number of flip flops available in the FPGA chip [19]. Therefore, another approach is used: If the local PN code input to the pair of early and late correlators is connected to the local PN code input to one of the correlators, for example, correlator 1, the phase error from that pair of correlators is 0 except when correlator 1 gives a high correlation that reflects the value of the decoded symbol. Thus if the values of the 8 versions of the PN code are summed up and correlated with the half-chip advanced and retarded versions of the incoming signal, the result should be the correct phase error for adjusting the master clock.

Table 4.6 V/O Pin Definitions of the 8-bit-3-stage Shift Register

\begin{tabular}{|l|l|}
\hline \multicolumn{1}{|c|}{ I/O Pin } & \multicolumn{1}{c|}{ Description } \\
\hline $\mathrm{d}<7: 0>$ & Signal input \\
\hline
\end{tabular}


Table 4.6 I/O Pin Definitions of the 8-bit-3-stage Shift Register

\begin{tabular}{|l|l|}
\hline \multicolumn{1}{|c|}{ L/O Pin } & \\
\hline $\mathrm{ce}$ & Clock enable \\
\hline $\mathrm{c}{ }^{\prime}$ & Clock input \\
\hline $\mathrm{clr}{ }^{\prime}$ & Reset \\
\hline $\mathrm{ql}<7: 0>$ & Late version of the incoming signal \\
\hline $\mathrm{qc}<7: 0>$ & Punctual version of the incoming signal \\
\hline $\mathrm{qe}<7: 0>$ & Early version of the incoming signal \\
\hline
\end{tabular}

A 4-input adder (Figure 4.16) is designed for summing up the $8 \mathrm{PN}$ codes in two groups. Since each PN code contains only 1's and 0's (corresponding to actual values of +1 's and -1 's), all the inputs to the 4-bit adder (a0, a1, a2, and a3) have a size of 1 bit and the maximum output is 4 . Thus the output can be sufficiently represented by a 3-bit number ( $\mathrm{y} 2$ y1 y0). To sum up all the 8 PN codes, the outputs of 2 4-input adders are summed by a 3-bit adder to yield a 3-bit number. In the schematic, however, a 4-bit adder is used in place of 3 bit adder mainly because the 4-bit adder is provided by the Cadence system as a macro and in this case it will be trimmed to a 3-bit adder automatically when the circuit is compiled for programming the FPGA.

The designs of the early and late correlators are very similar to the ones used for decoding data except that the incoming signal is correlated with the summed value of the 8 PN codes. A close examination of the PN codes reveals that only the possible values of the sum of the 8 PN codes for any particular chip are $7,6,5,4,3,2,1$ (corresponding to actual values of $+6,+4,+2,0$, $-2,-4,-6)$. Therefore, a module called 4_4_decode is designed to select the correct multiplicand to the incoming signal for each chip. As shown in Figure 4.17, \{a2 a1 a0 $\}$ is the 3-bit input for the summed value of the $8 \mathrm{PN}$ codes and $\mathrm{s}$ is the phase uncertainty of the incoming signal. The first output of the module, a0, is used to select whether the accumulation is an increment or decrement. The next four outputs, a1, a2, a3, a4, are used to activate the multiplicand to be multiplied to the 


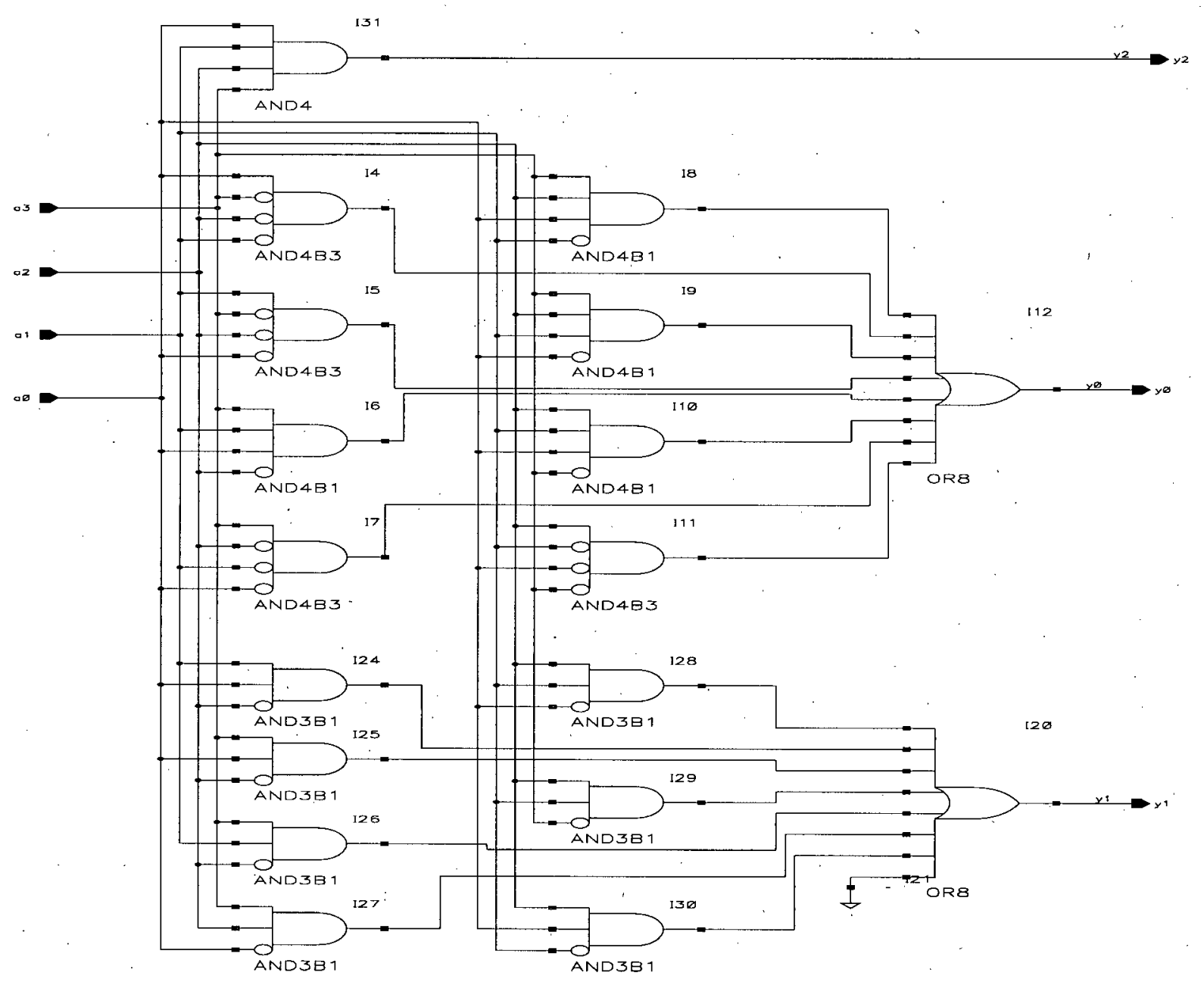

Figure 4.16 4-input Adder

incoming signal.

The schematics of the early and late correlators are shown in Figure 4.18 and Figure 4.19 respectively. They are identical except that the offset of the accumulator in the early correlator is much higher than that in the late correlator to eliminate the handling of negative numbers when taking the phase error value by subtracting the output of the late correlator from that of the early 


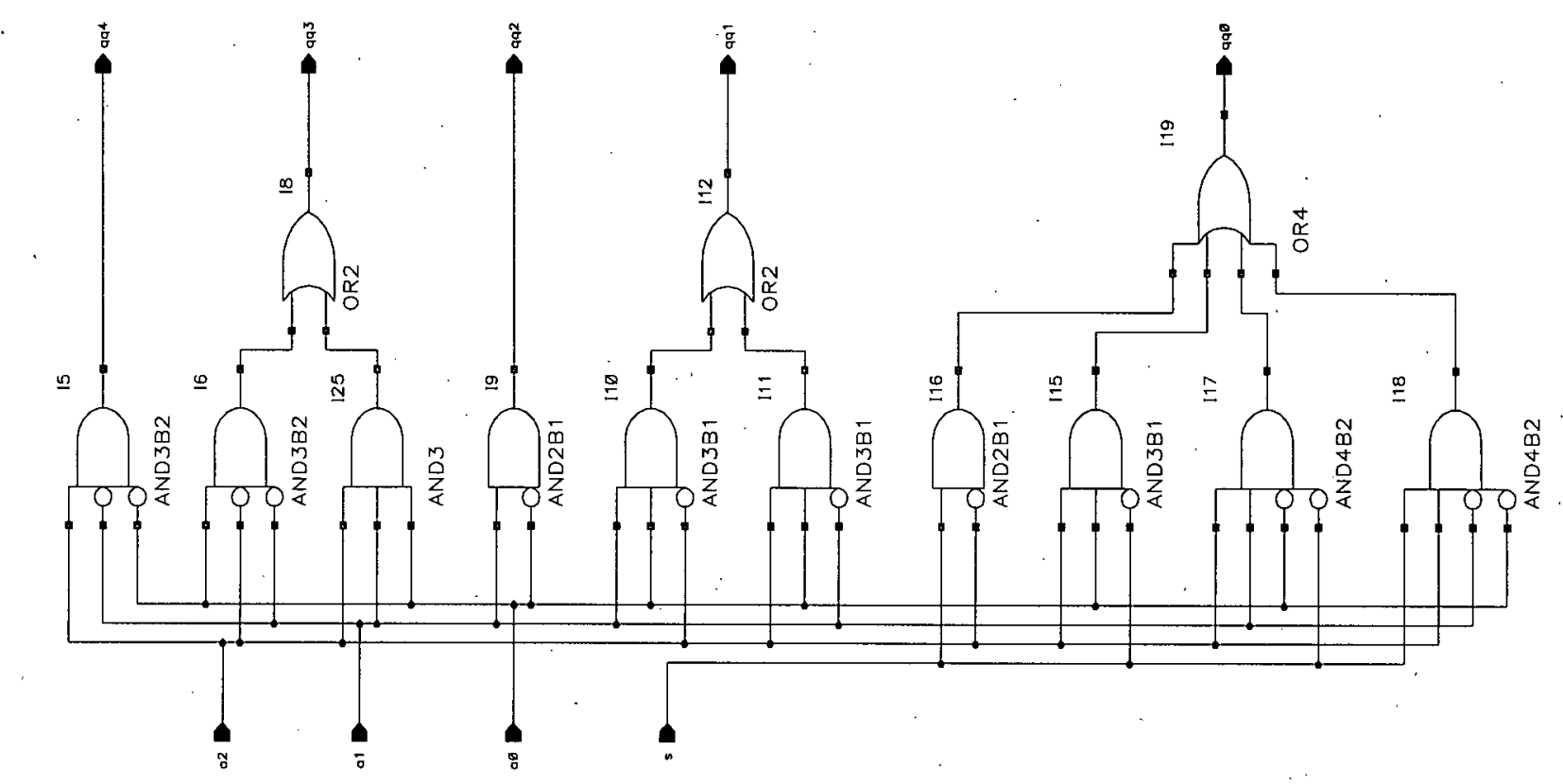

Figure 4.17 4-4-Decode Circuit

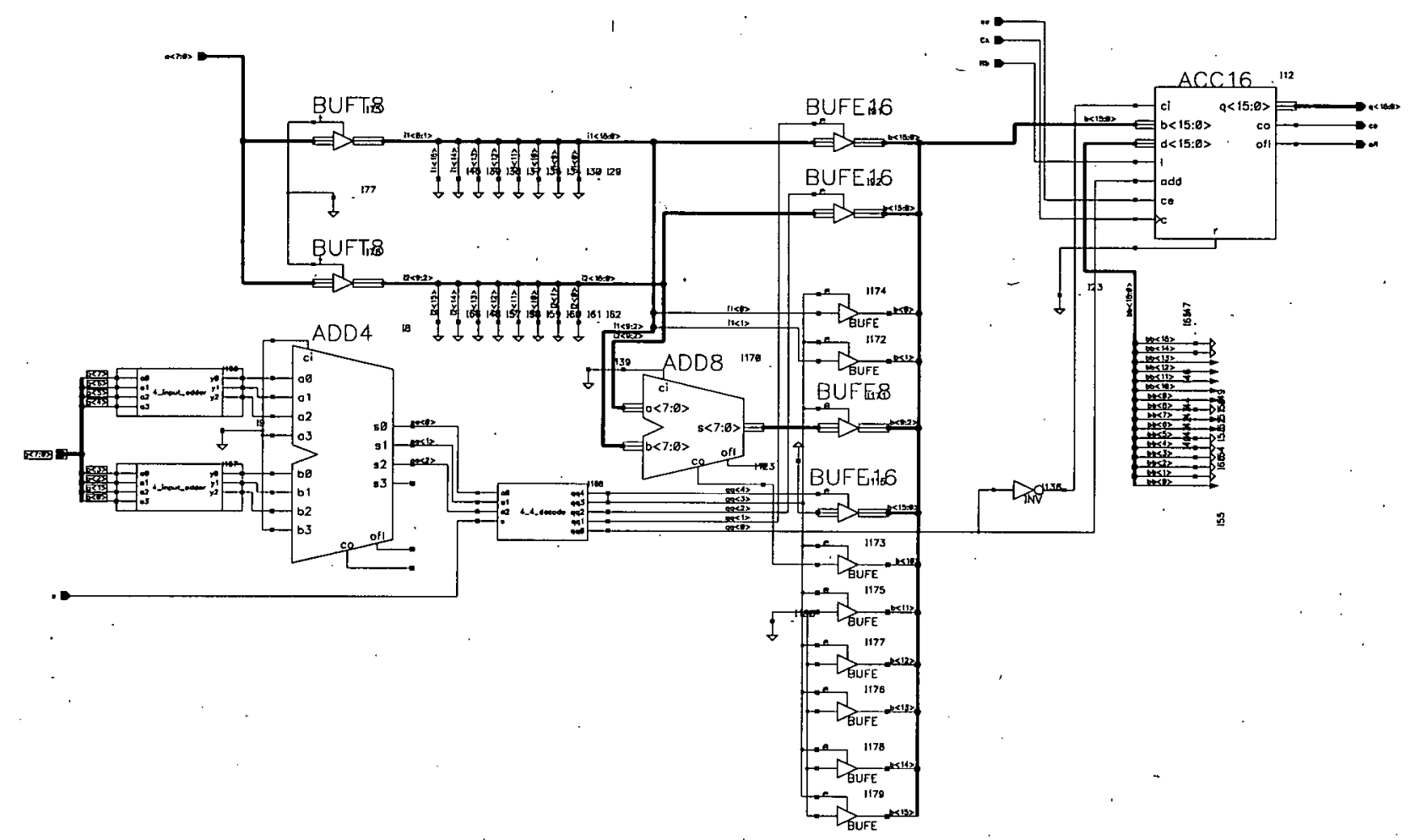

Figure 4.18 Early Correlator

correlator. This phase error is fed into the analog section of the decoder for adjusting the master 
clock of the system as described in the next sub-section.

Finally, the schematic of the CPSK decoder, without the analog section, is shown in Figure

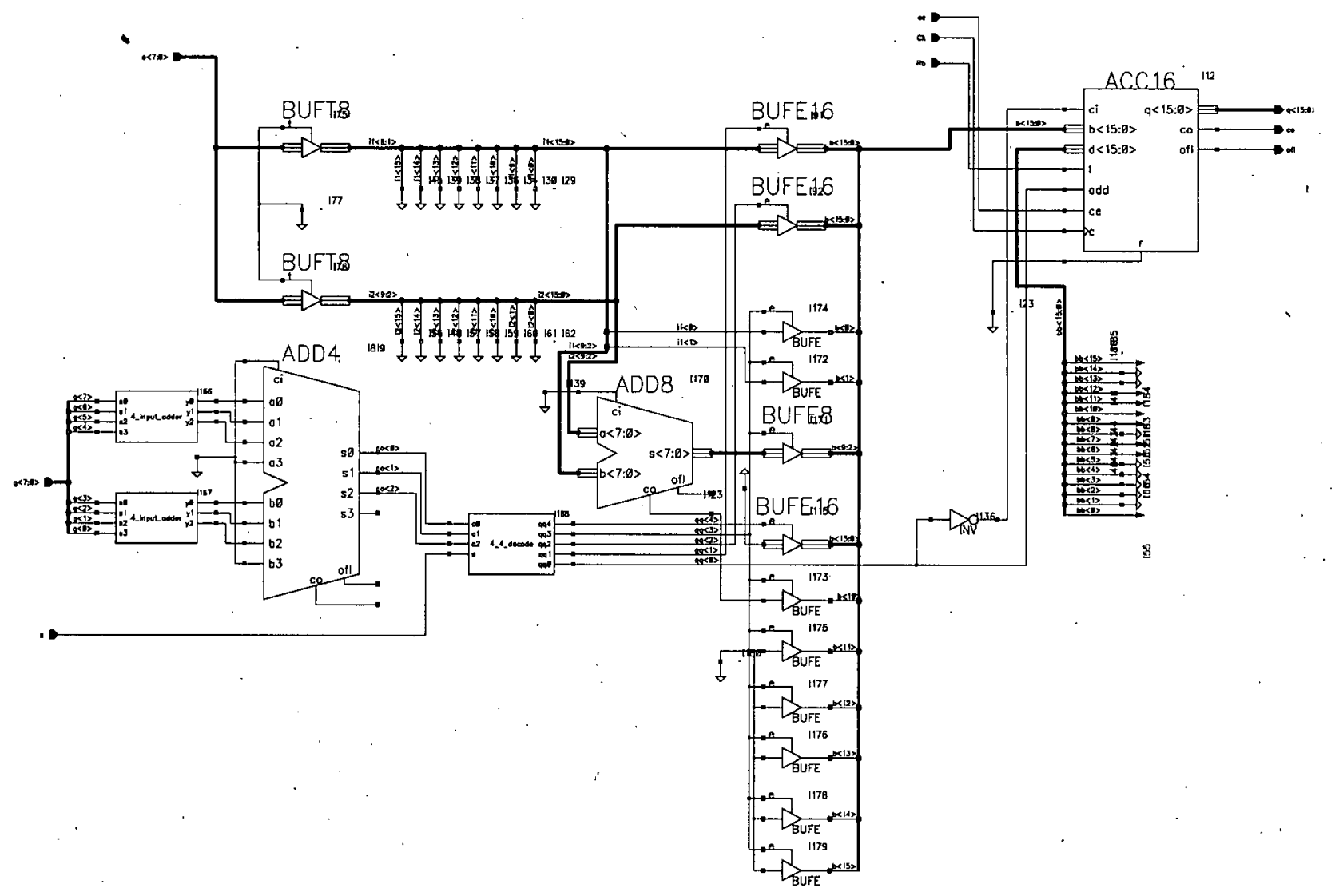

Figure 4.19 Late Correlator

4.20. The pin assignment and the function of each pin of the decoder implemented in the XC4010 FPGA is explained in detail in Appendix A.

\subsubsection{The Analog Section}

The major components in the analog section include an 8-bit D/A converter and a VCXO 


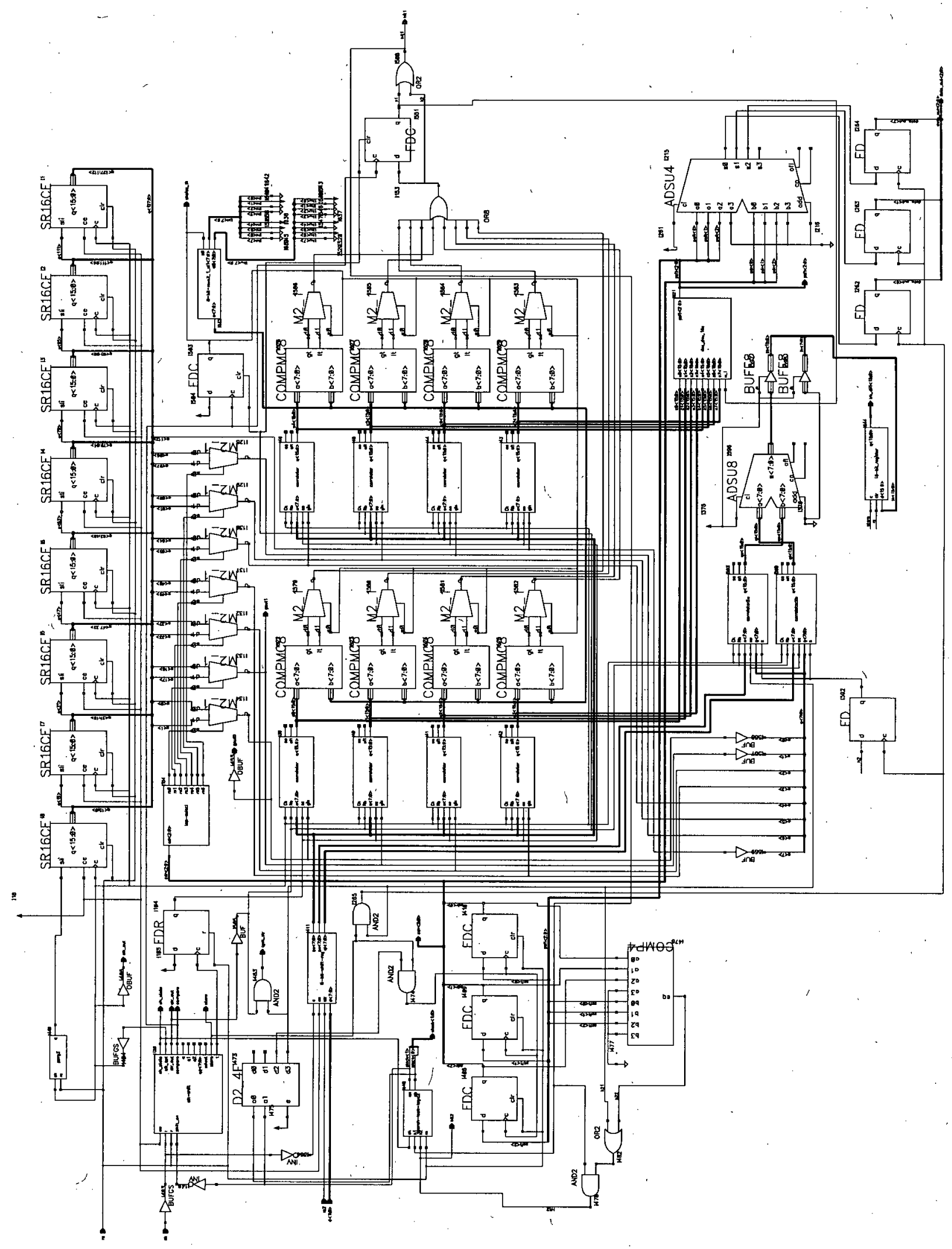


(Voltage Controlled Crystal Oscillator). As shown in Figure 4.21, the phase error from the pair of early and later correlators are converted to an analog voltage to control the frequency of the VCXO. The output of the oscillator provides the master clock signal. Two potentiometers, R5 and R7, are used to adjust the control range and the center frequency generated by the crystal oscillator.

The frequency range of the clock signal is calculated as follows. Assume that the nominal clock frequency is $16 \mathrm{MHz}$ and the length of the PN sequence is 127 chips. In order to let the system go through from the acquisition state to the lock state successfully (which at least requires the duration of 3 PN cycles), the clock signal should be stable enough so that the drifting is less than 1 chip in a duration of $3 \mathrm{PN}$ cycles. Thus the maximum drift of the clock signal with respect to the nominal value is $+/-1 / 3$ chip in each PN cycle. At nominal frequency, the duration of a PN cycle is:

$$
T_{d}=\frac{1}{16 M} \times 127 \times 2=15.875 \mu S
$$

If the drift is $1 / 3$ chip less in $1 \mathrm{PN}$ cycle, the chip duration becomes:

$$
T_{c}=\frac{T_{d}}{127-1 / 3}=125.329 n S
$$

Thus the frequency required to have 1 chip deducted is:

$$
f_{l}=\frac{1}{T_{c} / 2}=15.958 \mathrm{MHz}
$$

Similarly the frequency required to have 1 chip increased in a PN cycle is: 


$$
f_{u}=\frac{1}{T_{c}^{\prime} / 2}=\frac{1}{\frac{1}{16 M} \times \frac{127}{127+1 / 3}}=16.042 \mathrm{MHz}
$$

Therefore, the maximum frequency control range is $(15.958 \mathrm{MHz}, 16.042 \mathrm{MHz}){ }^{\prime}$

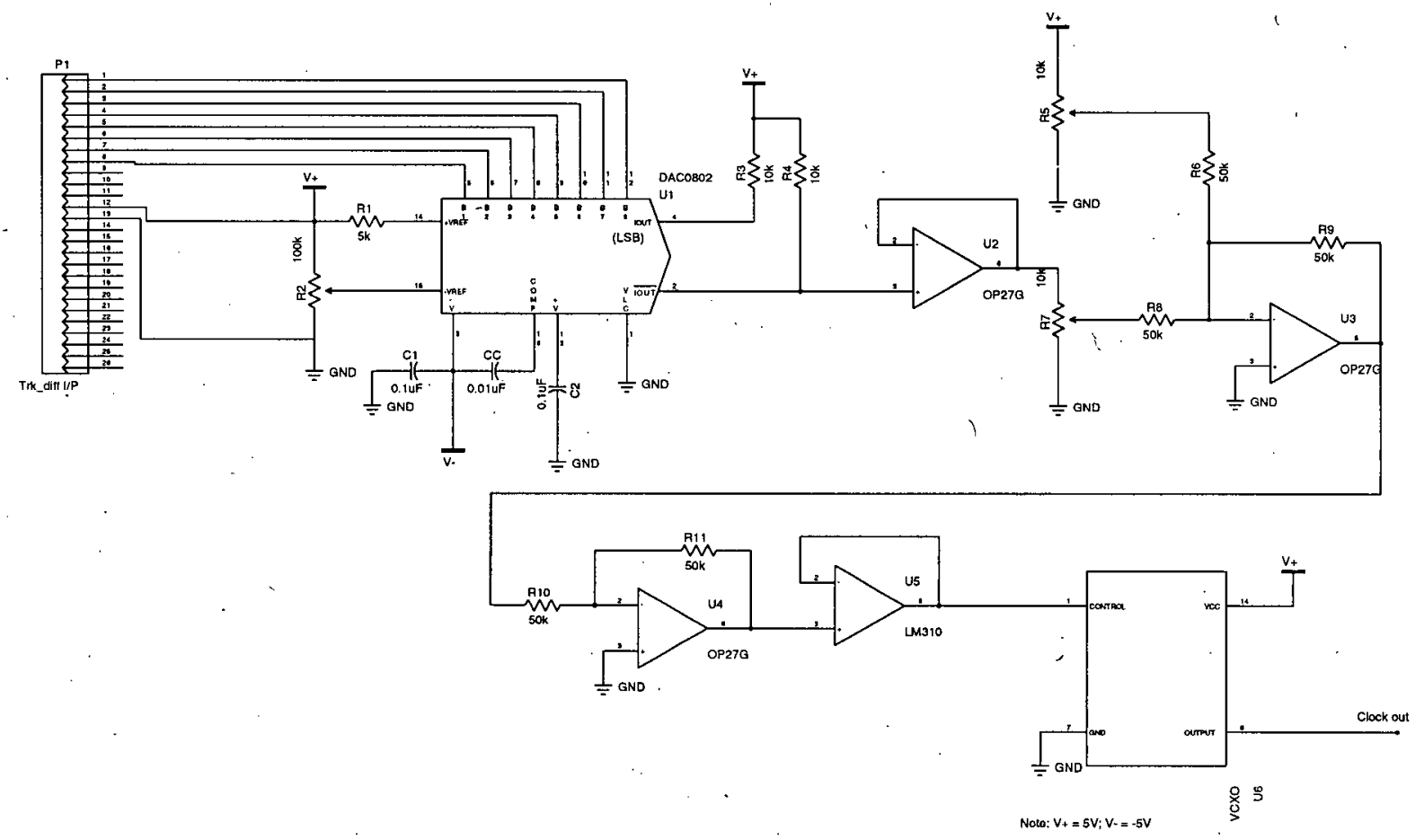

Figure 4.21 The Analog Section of the CPSK Decoder

It is important that the oscillator is stable enough such that the variation range is less than the range calculated above in order for the acquisition mode to work properly: Therefore, a Raltron VC7025-LZ-200-16.000MHz VCXO with frequency stability of $100 \mathrm{ppm}$ and a control range of $200 \mathrm{ppm}$ is chosen to generate the master clock signal.

\subsection{The PC Interface}

As explained in the Chapter 3, the major function of the PC interface is reading data and 
some status signals from the CPSK Decoder. The major components of the interface include a FIFO memory and some registers. Besides assigning the $\mathrm{I} / \mathrm{O}$ addresses for reading the data and the status signals from the decoder, two other I/O addresses are needed to set the mode of the FIFO and write data into the FIFO from the PC for testing. The assignment of the addresses is listed in Table 4.7.

Table 4.7 Addresses used by the PC Interface

\begin{tabular}{|c|c|}
\hline Function & Address (hex) \\
\hline FIFO Mode & 0301 \\
\hline Read Flag Status & 0301 \\
\hline Read/write Data & 0300 \\
\hline FIFO Reset & 0302 \\
\hline
\end{tabular}

The SN74ACT72241L FIFO memory provides separate clock inputs for reading and writing data so that both processes are independent of each other. The FIFO also provides some flags for indicating various status of the memory, as defined in Table 4.8 .

Table 4.8 Status Flags of the FIFO Memory

\begin{tabular}{|l|l|}
\hline Flag & Purpose \\
\hline FF (Full Flag) & The FIFO is full \\
\hline EF (Empty Flag) & The FIFO is empty \\
\hline AEF (Almost Empty Flag) & The FIFO is almost empty. The level of the emptiness can be programmed. \\
\hline AFF (Almost Full Flag) & The FIFO is almost full. The level of fullness can be programmed. \\
\hline
\end{tabular}

The mode is controlled by the REN1, WEN2/LD, and WEN1 pins on the FIFO chip which are set by the mode register. The assignment of these signals to the mode register bits is shown in Figure 4.22.

The detail circuit as well as the interface to the PC bus and the CPSK decoder are shown in 


\begin{tabular}{|cccccccc|}
\hline D7 & D6 & D5 & D4 & D3 & D2 & D1 & D0 \\
W & $\mathrm{X}$ & $\mathrm{X}$ & $\mathrm{X}$ & $\mathrm{X}$ & REN1 & WEN2/LD & WEN1 \\
W: Write from PC bus (0) or from decoder (1) & & \\
X: No connection
\end{tabular}

Figure 4.22 The Mode Register

the schematic in Figure 4.23. The detailed information about address decoding and register read/ write can be found in [23] and [24]. The programming considerations of the interface are discussed in the next section.

\subsection{The PC Control Software}

The PC control software is written for testing the BER or SNR of the system under AWGN and interference from another transmitter. The testing is just like a conventional BER analyzer in that a predetermined data pattern is sent from the transmitter. When the data is received from the CPSK decoder, they are compared with the pattern and the number of errors and the amount of data read is recorded and displayed. The algorithm of the software is shown in Figure 4.24.

When the program is started, the FIFO memory is cleared and the mode of the FIFO memory is set. Then the program continues to read data from the FIFO until the required number of errors is reached. The source code is written in C and is listed in Appendix B. 


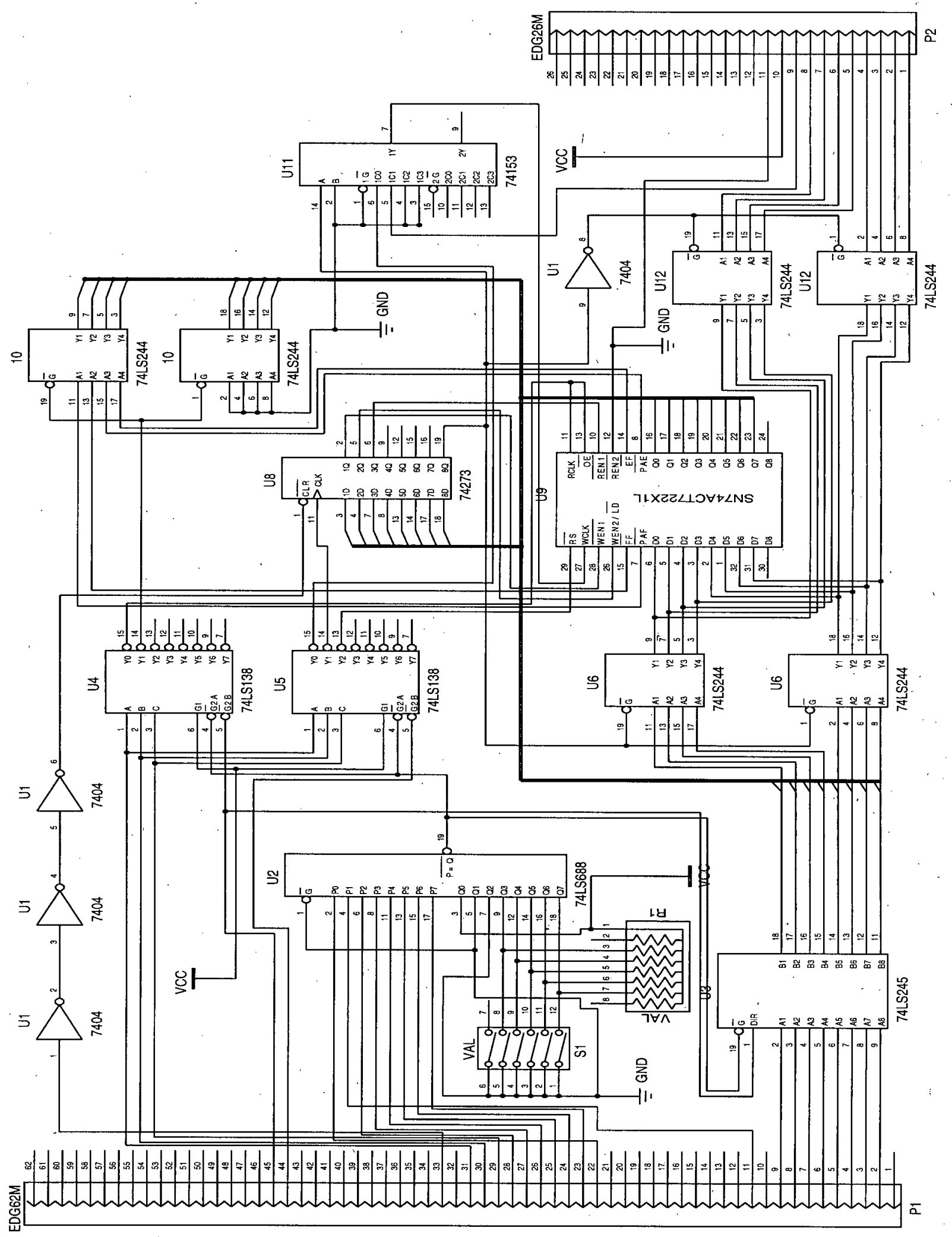

Figure 4.23 The Schematic of the PC Interface 


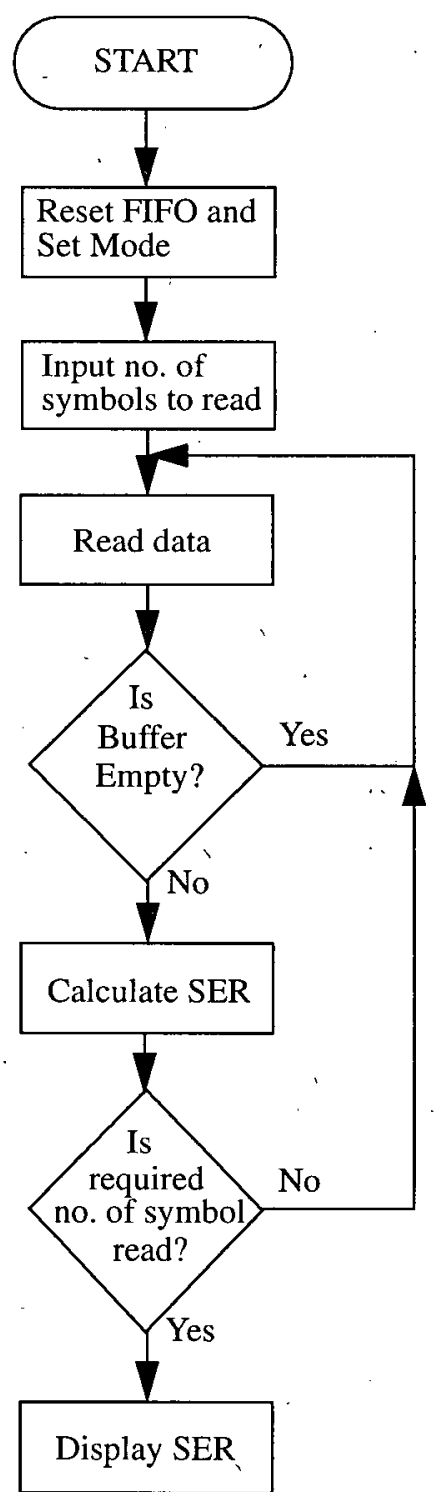

Figure 4.24 The Flow Chart of the PC Control Software 


\section{Chapter 5 Testing and Performance Evaluation}

\subsection{Testing}

Before measuring the performance of the CPSK receiver, some functional tests are needed to ensure proper operation of each part of the receiver. Unit testing on the IF demodulator, the CPSK decoder, and the PC interface were conducted which was then followed by a system test.

\subsubsection{Testing of the IF Demodulator}

The Costas loop needs to be tuned before it is tested. As seen in Figure 4.3, the circuit contains 3 potentiometers. $\mathrm{R} 1$ and $\mathrm{R} 2$ are responsible for adjusting the $\mathrm{DC}$ offsets to the $\mathrm{I}$ and $\mathrm{Q}$ inputs to the multiplier. The adjustments are made by feeding a $1 \mathrm{kHz}$ signal to each input and turning the potentiometer until the output contains no DC component. Then the $1 \mathrm{KHz}$ signal is removed and the loop is opened by disconnecting the I and Q inputs to the multiplier from the BPSK demodulator. At this time, the free running frequency (or the center frequency) is adjusted by R3 until the output frequency from the VCO is $140 \mathrm{MHz}$.

To test the functionality of the Costas loop, the output signals to the A/D converter are displayed on a Tetronix 2230 digital storage oscilloscope with the input signal from the DSP based transmitter [5]. If the Costas loop operates properly, one of the waveforms displayed on the scope should be the same PN sequence as sent by the transmitter. Then the demodulator is ready for system testing.

\subsubsection{Testing of the CPSK Decoder}

Since the decoder is designed using the Cadence system [20], verification on the logic functions by simulations can be done before downloading the circuit to the FPGA chip for real- 
signal testing. The Verilog simulation tools provided by Cadence is used for generating the input test patterns and capturing the signals at various points in the circuit. The detailed procedures in Verilog simulations can be found in [21]. Basally, a test script (which is called a test fixture) is written to specify the logic values of the input signals to the decoder at different time intervals. If the decoder works properly, the value of the decoded symbol should correspond to the phase shifts of the input PN sequence generated by the test script.

After simulation tests have been passed, the circuit is compiled into a bit stream configuration file which is burnt into an EPROM attached to the FPGA chip for in-circuit testing. A Tektronix 3001GPX logic analyzer is used to monitor the inputs and outputs of the chip. To isolate the effect on the IF modulation/demodulation process, the input to the decoder is directly connected to the output of the CPSK encoder part of the transmitter. The transmitter is then programmed to send out different data patterns and compared with the decoded symbol captured by the logic analyzer.

\subsubsection{Testing on the PC Interface}

The testing of the PC interface is straight forward. A piece of software is used to write various quantities of data with different size to the FIFO memory, read the data back from the FIFO memory, and check if there are any errors. The tests are performed by using functions test() and test2() as shown in Appendix C.

\subsubsection{System Testing}

The setup for system testing is shown in Figure 5.1. The CPSK transmitter produces a 3 $\mathrm{dBm}$ DSB-SC signal with carrier frequency at $140 \mathrm{MHz}$ and is attenuated by $30-50 \mathrm{~dB}$ using the 
KAY 437A step attenuator. The signal is then summed with AWGN by Mini-Circuits ZSC-2-1 0 degree summer/splitter.

The AWGN produced by W \& B white noise generator has a bandwidth from $6 \mathrm{kHz}$ to 25 $\mathrm{MHz}$ and so a LO signal with frequency near $140 \mathrm{MHz}$ is needed to bring the AWGN to cover the frequency range of the transmitted signal. This is done by mixing the AWGN with the HP 8656B signal generator using the Mini-Circuits MCL SBL-1 frequency mixer.

The mixed signal is then amplified by $28 \mathrm{~dB}$ with the Mini-Circuits ZHL-2-8 RF amplifier before feeding into the IF demodulator of the CPSK decoder for BPSK demodulation. The demodulated signal is then decoded by the CPSK decoder and finally read into the computer.

The system is tested by sending different data to the receiver and checking the data collected by the PC at different noise levels. The receiver should give no error when the noise is absent and the symbol error rate (SER) or bit error rate (BER) should increase with the amount of noise added to the system. Then detailed performance measurements are conducted as shown in the next section.

\subsection{Experimental Setup}

The setup for measuring the performance of the CPSK receiver is the same as the one used for system testing in Figure 5.1. The SNR of the signal is measured with the Tektronix 497P spectrum analyzer. To measure the BER or SER, simple signal patterns are generated from the transmitter. The receiver operates like a BER analyzer and counts the number of symbols that do not match the pre-defined pattern.

To evaluate the performance with jamming from another transmitter, some modification 


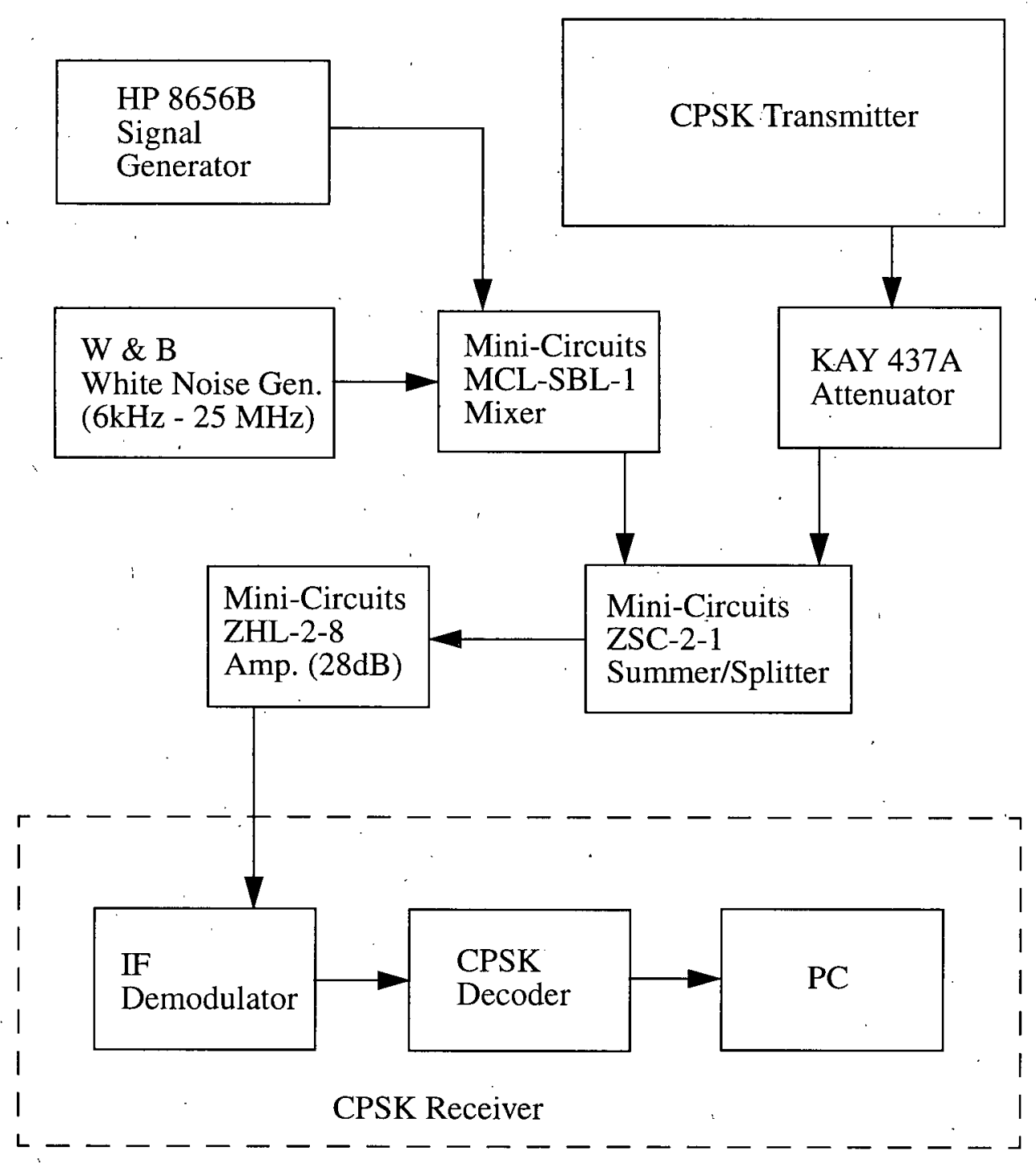

Figure 5.1 Experimental Setup

on the setup is needed. An additional Mini-Circuits ZSC-2-1 Summer/Splitter is inserted into the path between the $28 \mathrm{~dB}$ amplifier and the IF demodulator to mix with the signal from another CPSK transmitter.

\subsection{Performance of the CPSK Receiver Under AWGN}

By the addition of the Costas loop, the receiver can be operated independently (i.e., 
without the need of synchronized carrier signal from the transmitter.) However, it is of interest to measure the performance of the receiver with the carriers of both the transmitter and receiver perfectly synchronized because the measurements represent the upper limit of the performance of the receiver employing the Costas loop for carrier synchronization. The performance of the receiver with perfectly synchronized carriers and using the Costas loop are discussed in the following two subsections.

\subsubsection{Performance with Perfectly Synchronized Carriers}

The curve of the experimental BER versus SNR of the receiver using perfectly synchronized carriers is plotted in Figure 5.2. For comparison purposes, the ideal curve from theoretical computations is also included in the figure.

The ideal bit error rate is given by Equation (2.22) in Chapter 2. Since we are plotting BER against SNR, the conversion from SER to BER is needed. From [4], the conversion is given by:

$$
B E R=S E R \times\left(\frac{2^{(M-1)}}{2^{M}-1}\right)
$$

The results is plotted in Figure 5.2: Each point on the experimental curve represents the average of 10 measurements. Assume that the data is normally distributed (approximations to students' t distribution with degree of freedom 9), then the $95 \%$ confidence interval is $\frac{1.96 \times \sigma}{\sqrt{10}}=0.62 \sigma$ where $\sigma$ is the standard deviation of the data [25]. An error bar is therefore 
added to each point to indicate that the actual BER is bound by each error bar with $95 \%$ of confidence. When the BER is high (at the order of $10^{-3}$ ), the deviation of the measured values from the corresponding theoretical value are as small as $0.25 \mathrm{~dB}$. As the $\mathrm{S} / \mathrm{N}$ increases, the performance degradation gradually increases to about $0.85 \mathrm{~dB}$ at a BER of $10^{-6}$. Three factors contribute to the degradation: Since digital correlators with filter instead of optimal correlators are used to correlate the incoming signal, the correlation errors contribute to the performance degradation; Secondly, an 8-bit D/A converter is used to convert the quantized error signal to control the VCO which means that the local PN code may never be aligned perfectly with the incoming signal and as a result, the performance is degraded; Finally, the noise inherent in the receiver contributes to the degradation especially when the $\mathrm{S} / \mathrm{N}$ is high. The performance of the system employing synchronized carriers represents the upper limit of the performance when the Costas loop is employed. In the following subsection, the performance using the Costas loop is compared with this upper limit.

\subsubsection{Performance with Costas Loop}

The performance curve of the receiver with Costas loop implemented is shown in Figure 5.3. When compared to the curve with perfectly synchronized carrier, the performance is degraded by approximately $1.5 \mathrm{~dB}$ at $\mathrm{BER}=10^{-3}$ and $10^{-6}$, and $0.85 \mathrm{~dB}$ at $\mathrm{BER}=10^{-4}$. At low $\mathrm{S} /$ $\mathrm{N}$, the degradation is largely due to the instability of the Costas loop: The oscillating frequency of the loop is dependent on the error signal. As the noise is increased, the error signal is changed more rapidly and so the frequency generated by the VCO will move around the center frequency more frequently. Since the VCO becomes less stable, the PN sequence contained in the demodulated signal for decoding will be less accurate. After correlating with the local PN code, the 


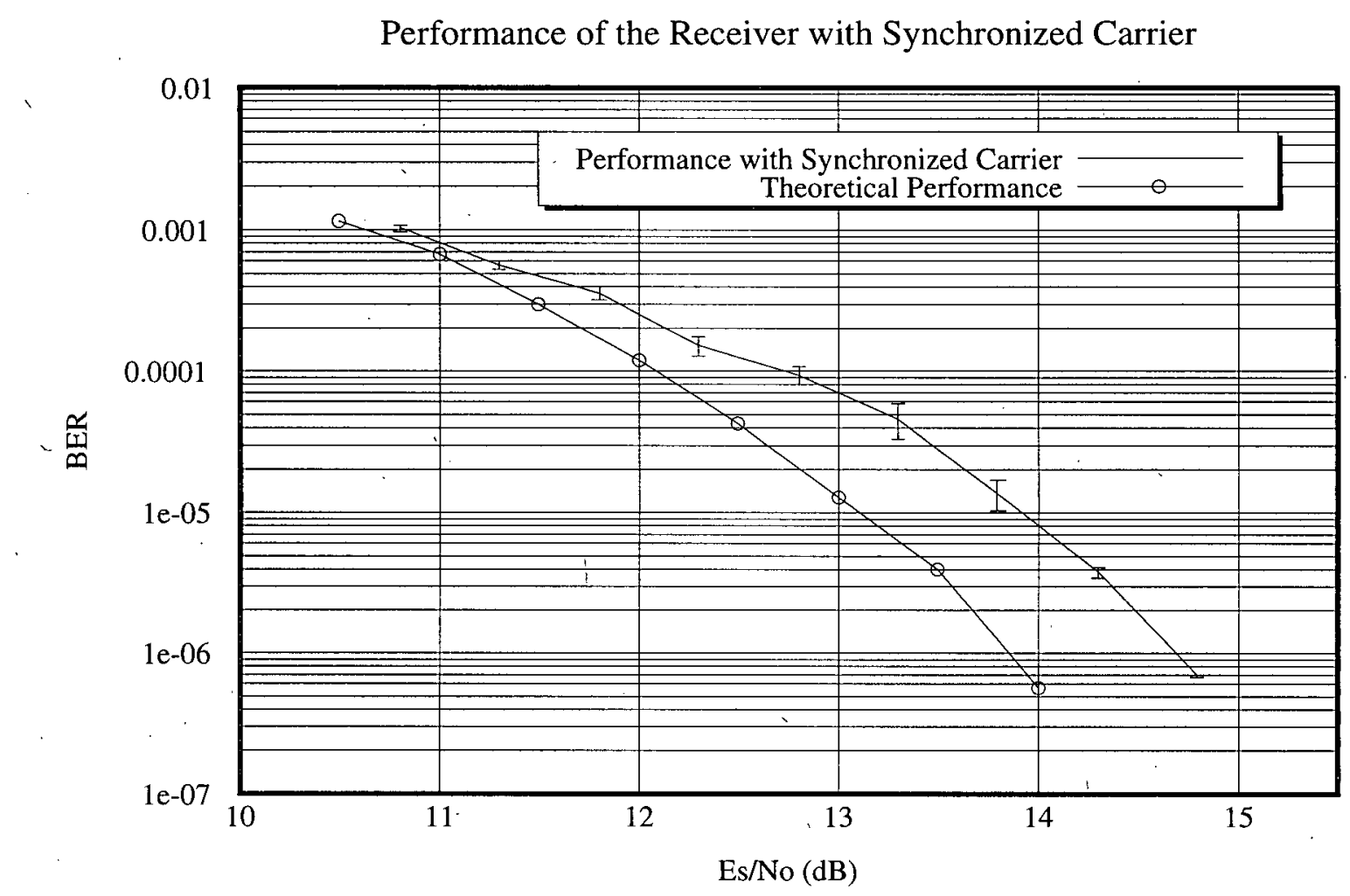

Figure 5.2 Performance of the Receiver with Synchronized Carrier

correlated value of the data portion of the received signal will be decreased and as a result, the symbol error rate is increased. As $\mathrm{S} / \mathrm{N}$ is increased, the Costas loop becomes more stable so that the BER is decreased. However, when the $\mathrm{S} / \mathrm{N}$ is further increased, the deviation increases again because at this $\mathrm{S} / \mathrm{N}$ level, the intrinsic noise (mostly contributed by harmonics in the Costas loop and noise picked up by the BPSK demodulator) in the receiver becomes significant.

\subsection{Performance of the CPSK Receiver With Jamming From Another CPSK Transmitter}

The performance is measured when the transmitter is sending pre-defined patterns while the jammer is sending a-purely zero pattern. The carriers are generated by 2 separate RF generators. This makes the jamming a realistic situation where the transmitter and jammers are not 


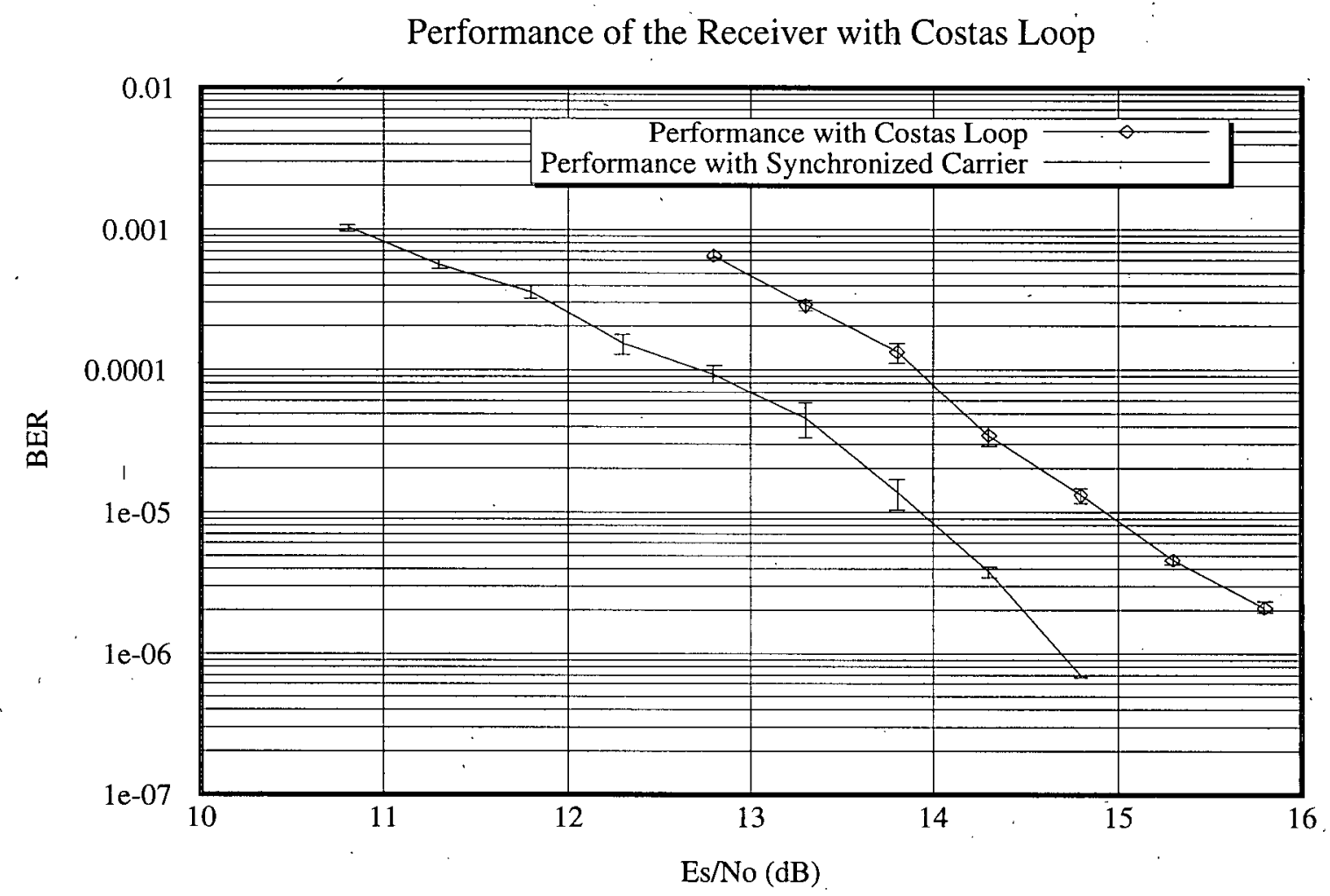

Figure 5.3 Performance of the Receiver with Costas Loop

synchronized. The PC compares the receiver decoded values with the pre-defined pattern to quantify the effect of the jammer on the BER. Four sets of measurements at SNRs of 12.8, 13.8, 14.8, and $15.8 \mathrm{~dB}$ were taken with different jamming levels and the respective BER curves are plotted in Figure 5.4. In general, the BER increases with the jamming level and as the S/N decreases, the system is more tolerant to the jamming signal. When the jamming signal is absent, the BER is around $2.5 \times 10^{-6}$ at an SNR of $15.8 \mathrm{~dB}$ as shown in Figure 5.3. With the introduction of the jammer with JSR at $-8.5 \mathrm{~dB}$, the SER is raised to $2.5 \times 10^{-3}$. Any further increase in jamming signal will make the decoder unable to maintain tracking. 


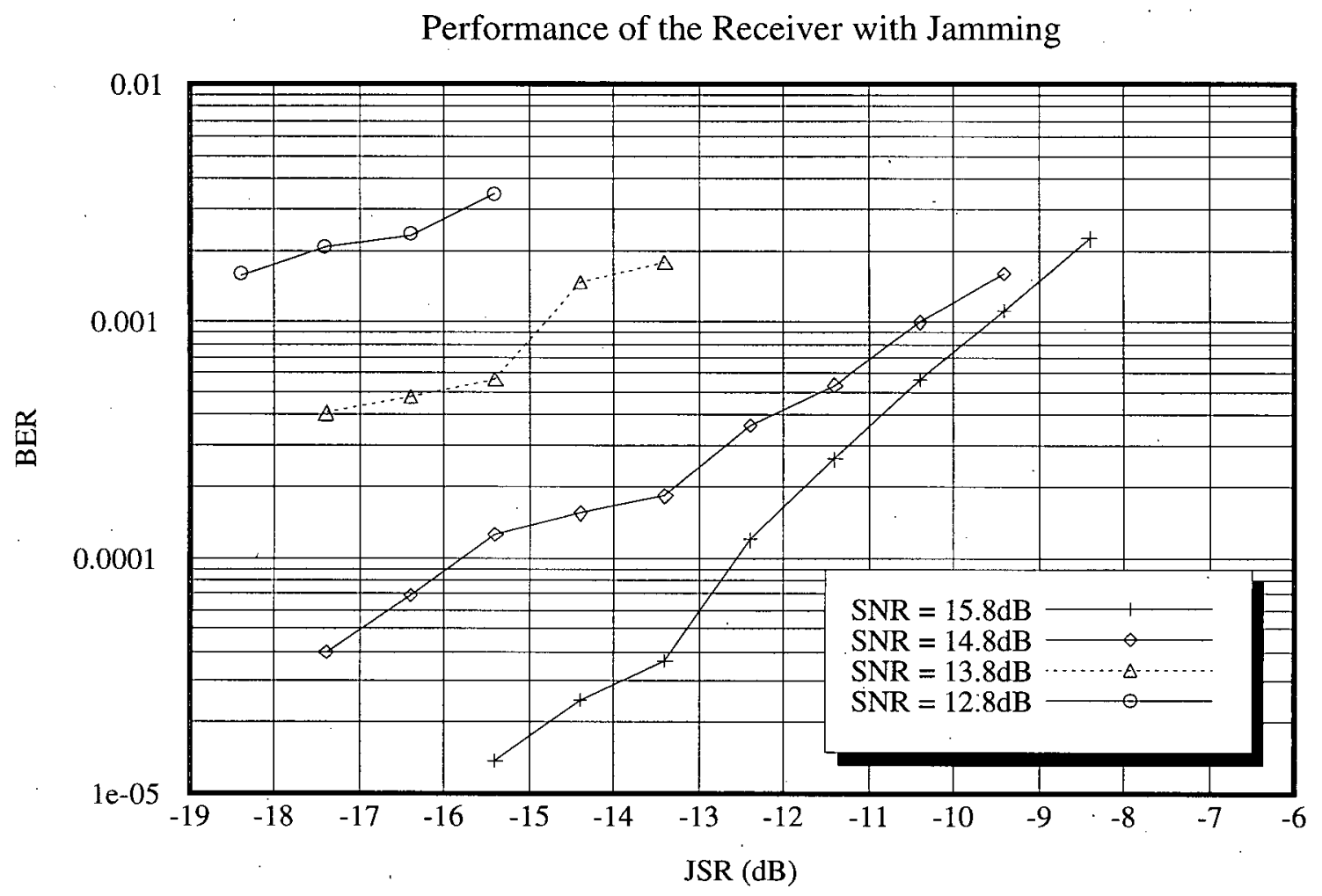

Figure 5.4 Performance of the Receiver With Jamming From Another Transmitter

To justify the performance degradation, note that the carrier phases are not synchronized, so that the BPSK demodulates both signals as shown in Figure 5.5. Error terms contributed by transmitters (the term with $A_{1}$ ) and the jammer (the term with $A_{2}$ ) are fed into the loop filter to control the frequency of the VCO. If the jammer is absent, the output is synchronized with the transmitter so that $\phi_{1}$ is approaching 0 and the VCO frequency is locked to the carrier frequency of the transmitter. If the jammer is present, the VCO will run at a frequency so that both error terms are minimized. Since the carrier phases are not synchronized, the VCO frequency varies with time and so the correlation of the demodulated signal with the local PN code is decreased and is more prone to noise. 


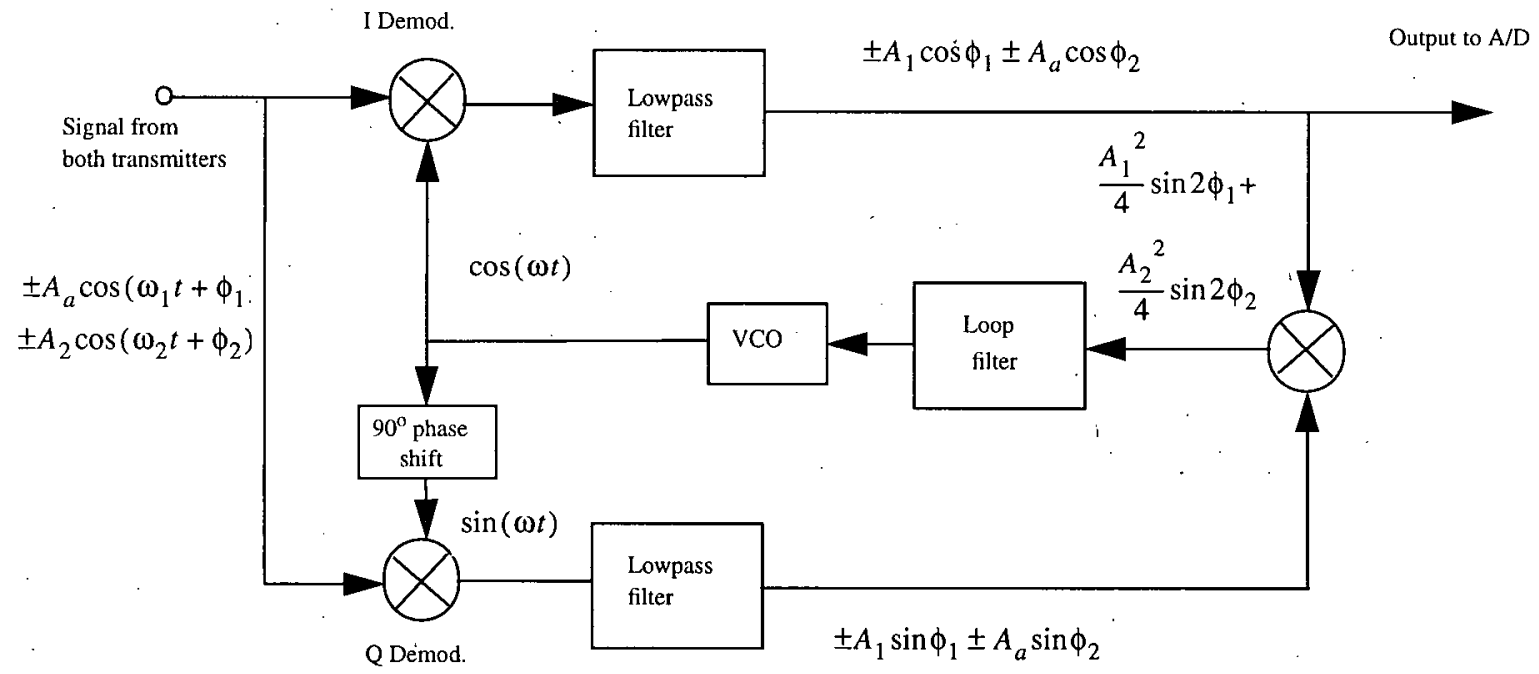

Figure 5.5 Costas Loop Demodulator With Signals From Both Transmitters 


\section{Chapter 6 Design Scalability of the FPGA Based Decoder}

The design of the CPSK decoder presented in Chapter 4 represents one possible configuration for decoding data with PN code length of 127 and symbol size of 3 . The decoder needs to be reconfigured if it is to decode data with different PN code lengths and symbol sizes. Since it,is implemented in a Xilinx 4010 chip which can be re-programmed from time to time, the decoder can be easily configured to decode data with another PN code length and symbol size by loading another data file that describes the circuit for decoding that type of data. However, the number of configurations that can be fit into the FPGA chip is limited by the size of the chip. Before we can modify the decoder with different configurations to fit into the chip efficiently, The basic architecture of the FPGA chip needs to be studied.

Also, the data rate of the decoder is dependent on the running frequency of the master clock to the FPGA chip. The maximum running frequency of the chip limits the maximum throughput as discussed in Section 6.3.

\subsection{Overview of the FPGA Chip}

The Xilinx FPGA (field programmable gate array) includes three major configurable elements: configurable logic blocks (CLBs), input/output blocks (IOBs), and interconnections. The CLBs provide the functional elements for constructing the users' logic. The IOBs provide the interface between the package pins and internal signal lines. The interconnections provide routing paths to connect the inputs and outputs of the CLBs and IOBs onto the appropriate networks. Customized configuration is established by programming internal static memory cells that determine the logic functions and interconnections implemented in the device. The details of CLBs, IOBs, and interconnections can be found in [19]. The CLBs are briefly discussed as 
follows:

The simplified structure of a CLB is shown in Figure 6.1. Each CLB contains two independent 4-input function generators and a pair of flip-flops and they can be accessed by thirteen CLB inputs and 4 CLB outputs. These inputs and outputs are connected to the interconnect resources outside the block. Each of the function generators (with inputs F1-F4, G1-G4 and outputs F', G') can be used to implement any 4-input Boolean function by means of memory look-up table. Therefore, the propagation delay of the CLB is not affected by the kind of Boolean function being implemented. F' and G' are connected to a 3-input function generator (with the third input $\mathrm{H} 1$ connected outside the block). Again this function generator can implement any 3input Boolean function. The outputs $Y$ and $X$ can be used to access the signals $G^{\prime}, H^{\prime}$ and $F^{\prime}, H^{\prime}$, respectively from the function generators. Therefore each CLB can be used to implement any 2 independent functions with up to 4 variables, or, any single function of five variables, or any function of four variables together with some functions of five variables, or even some functions of up to nine variables.

Two D-type flip-flops are used as the storage elements in the CLB and they share the same clock (K), clock enable (EC), and asynchronous set or reset (S/R) inputs. Each of the (S/R) inputs can be configured as a set or reset input independently by the S/R controls. Each flip-flop can be triggered on either the rising or falling clock edge and the data input (D) of each flip-flop can be programmed to accept signals $\mathrm{F}^{\prime}, \mathrm{G}^{\prime}$, and $\mathrm{H}^{\prime}$ from the function generators or the direct in (DIN) block input. The outputs of the flip-flops exit at pin XQ and YQ respectively.,

The F' and G' function generators in each CLB also contain some dedicated arithmetic logic (not shown in the figure). for the fast generation of carry and borrow signals so that the 


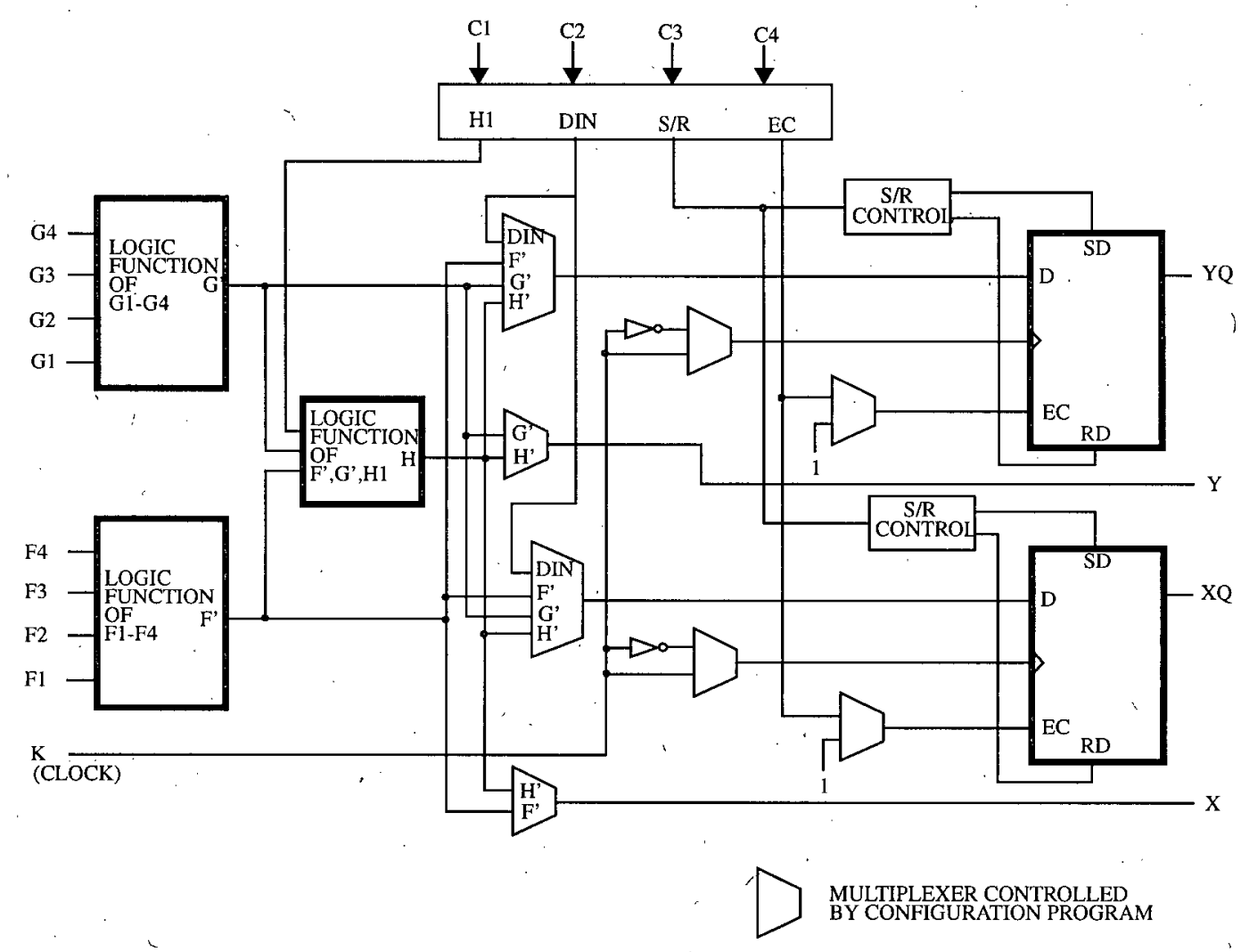

Figure 6.1 Simplified Block Diagram of a CLB

performance and the efficiency of adders, subtracters, accumulators, comparators and counters can be greatly enhanced. The inputs $\mathrm{C} 1-\mathrm{C} 4$ of the CLB can be mapped to the four internal control signals (H1, DIN, S/R, and EC) arbitrarily.

Since the flip-flops and function generators in the CLB can be treated as separate items, the inputs and outputs can freely swap positions within a CLB to provide the same functions, and the CLB is symmetrical, these attributes make the CLB highly flexible for implementing a design with ease of routing and high packing density.

\subsection{Design Scalability}

The CPSK decoder presented in Chapter 4 utilizes $98 \%$ ( 780 of 800 ) of the function 
generators and $40 \%$ (317 of 800) of the flip-flops which represents the design limit of the CPSK decoder in terms of the PN code length and the symbol size when a Xilinx 4010 FPGA is used. The basic design of the decoder for different PN code lengths and symbol sizes are the same (see Figure 4.5) except the number of correlators used. However, since the current design is optimized for decoding PN codes of length 127 and symbols size of 3 , the flexibility of the schematic for decoding codes of other PN code lengths and symbols sizes is limited.

If the decoder is used to decode signals with other PN code lengths and symbol sizes, modifications on some of the modules are needed. It is easy to modify each major component for decoding different codes. Thanks to the re-programmability of the FPGA chip, schematics of different configuration can be stored in separate data files and a switch of the configuration can be simply done by downloading the corresponding data file into the chip [22]. The following subsections outline the modifications of each component that have to be made for different configurations.

\subsubsection{The Digital Correlator}

For a $\mathrm{PN}$ code length of $\mathrm{G}$ and a data input from the $8 \mathrm{bit} \mathrm{A} / \mathrm{D}$ converter, the maximum and minimum correlated values (Cmax and $\mathrm{Cmin}$ ) are given by $+255 \mathrm{G}$ and $-255 \mathrm{G}$, respectively. As shown in Section 4.2.2, an offset equal to $\mathrm{ICminl}$ is added to eliminate the handling of negative numbers. Therefore, the accumulator should be designed such that it can accumulate values up to at least 510G. Table 6.1 shows some examples of the sizes of the accumulator needed with different $\mathrm{G}$ for 8 bit inputs. In general, for a data input from an $\mathrm{x}$ bit $\mathrm{A} / \mathrm{D}$ converter, the accumulator should be designed to handle numbers up to $2 \times 2^{x} \times G=2^{(x+1)} G$. Also, the offset to the accumulator needs to be re-calculated as described in Section 4.22. Obviously, this module is independent 
of the symbol size to be decoded.

Table 6.1 Size of Accumulator Needed According to G

\begin{tabular}{|c|c|c|}
\hline $\mathbf{G}$ & $\mathbf{k}$ & Bit Size of the Accumulator \\
\hline 15 & 4 & 13 \\
\hline 31 & 5 & 14 \\
\hline 63 & 6 & 15 \\
\hline 127 & 7 & 16 \\
\hline 255 & 8 & 17 \\
\hline
\end{tabular}

\subsubsection{The PN Code Generator with Shift Register}

The possible lengths of PN codes are given by $G=2^{N}-1$, where $N$ is a positive integer. In actual implementation, $\mathrm{N}$ and $\mathrm{N}-1$ also represent the number of flip-flops and 2-1 multiplexers needed in the PN code generator. The connection between the flip-flops and multiplexers are the same and only the feedback path consisting of AND and OR gates needs to be adjusted. The connection of the feedback path is shown in detail in [1]. The length of the shift register should also be modified such that the number of flip-flop stages is equal to $\mathrm{G}$.

\subsubsection{The Tap Select Module}

The design of this module depends on the symbol size used and is independent of the PN code length. Figure 4.10 shows the design for decoding 3 bit symbols. If a smaller symbol size is used, the higher order input and output pins can be grounded and remain unconnected respectively. The XACT compiler for the FPGA will automatically trim off the unused pins and the useless gates in the circuit will not occupy the resources in the FPGA chip. If a larger symbol size is used, however, the circuit needs to be redesigned as follows. Let the PN code length be G and the symbol size be $\mathrm{s}$. Then the number of taps to the correlators needed is $\mathrm{N}=2^{\mathrm{s}}$ and the number . 
of 2:1 multiplexers needed is $\mathrm{N}-1$ (for the last tap to the correlator does not need to swap positions). Define the control inputs to the multiplexers mux $(x)$ as $t(x)$ where $x=1 \ldots N-1$, the inputs to $\operatorname{mux}(x)$ as $i(x, 0)$ and $i(x, 1)$ where the code phase of $i(x, 1)$ lags that of $i(x, 0)$ by 1 chip. Let the output of the multiplexer $\operatorname{mux}(\mathrm{x})$ be connected to correlator $\mathrm{c}(\mathrm{x})$, and the address of the correlator $c(x)$ be a s-bit binary number $a(x)=\left\{a_{s} a_{s-1} \ldots a_{1}\right\}$. When $a(r)$ is the address of the correlator $\mathrm{c}(\mathrm{r})$, the control inputs $\mathrm{t}(1) \ldots \mathrm{t}(\mathrm{r}-1)$ must be set to 1 so that the multiplexers $\operatorname{mux}(1) \ldots$ $\operatorname{mux}(\mathrm{r}-1)$ select the input $\mathrm{i}(\mathrm{x}, 1)$. Then values for $\mathrm{t}(1) \ldots \mathrm{t}(\mathrm{N}-1)$ are tabulated against $\mathrm{a}(\mathrm{x})$. After converting $\mathrm{t}(\mathrm{x})$ to logic functions in terms of $\mathrm{a}(\mathrm{x})$ by using Karnaugh map, the corresponding combinational logic circuits can be implemented.

\subsubsection{The Decision Device}

The size of the decision device depends on the bus size of the output of the digital correlator and the symbol size. Normally, if the bus size is 16 bit, the digital comparators and multiplexers should be 16-bit wide. However, the lower order bits can be ignored if the PN code length is long enough and if there is not enough resource in the FPGA. The decision device designed in Chapter 4 illustrates the compromise of using 8-bit devices to compare 16-bit signals. The circuit in Figure 4.11 shows the circuit for making decisions among 8 values that corresponds to the decoding of 3-bit symbols. If a different symbol size is used, the comparator/multiplexer patterns need to be trimmed off according to the number of inputs to the device, and the decoding circuit on the right hand side of the circuit needs to be redesigned. The design of the decoding circuit can easily be done by setting up a truth table for each of the output pins followed by solving the logic equations using Karnaugh maps [9]. 


\subsubsection{The Clock Shift Module}

As mentioned in Chapter 4, the clock shift module provides the clock signal to the PN code generator and shift register, and 4 timing signals (compare, ch_state, store, and clear) to the decoder. The major components in this module include a counter, multiplexers, flip-flops and AND gates. In order to achieve half-chip shifting, a counter capable of counting up to a value corresponding to double the length of the PN code is needed. For example, if the length of the code is 127 , then a counter able to count up to 254 is needed and an 8 -bit counter is chosen. The 4 timing signals occur half a chip apart in the last 2 chips of the PN code. AND gates with some multiplexers and flip-flops are used to detect the 4 half-chips at the end of the code. The number of inputs to the AND gates depends on the size of the decoder. For example, if a 8 bit decoder is used, 8-input AND gates will be used. As with the digital correlator, the module is independent to the symbol size chosen.

\subsubsection{The Search Lock Logic}

Since the search lock logic is completely independent to the symbol size as well as the PN code length, there is no need to change the circuit.

\subsubsection{The Tracking Loop}

The tracking loop consists of 1 early correlator, 1 late correlator, a subtractor, a D/A converter, and a VCO. The correlators and the subtractor need to be changed according to the PN code length and symbol size used. It is the late and early correlators that take the most effort to accommodate the change. As shown in Figure 4.17 and Figure 4.18, each correlator can accommodate a maximum of 8 shifted PN codes corresponding to the decode of 3-bit symbols. The four-bit adder and the 4-input adders can be easily trimmed or expanded depending on the 
symbol size to be decoded. However, the 4-4 decode circuit has to be redesigned from scratch if another symbol size is used. The design of the circuit is outlined as follows:

Let the symbol size to be decoded be $\mathrm{k}$ and so $\mathrm{N}=2^{\mathrm{k}}$ shifted $\mathrm{PN}$ codes are used. After the summation of all the $\mathrm{N}$ codes at a time, (the codes contains only +1 and -1 values), the possible values are listed in the following table.

Table 6.2 Possible Values of the Sum of PN Codes

\begin{tabular}{|c|c|}
\hline No. of $+\mathbf{1}$ 's at $\mathbf{1}$ time & Sum \\
\hline $\mathrm{N}$ & $\mathrm{N}$ \\
\hline $\mathrm{N}-1$ & $\mathrm{~N}-2$ \\
\hline$\ldots$ & $\ldots$ \\
\hline $\mathrm{N} / 2$ & 0 \\
\hline$\cdots$ & $\cdots$ \\
\hline 1 & $-\mathrm{N}+2$ \\
\hline 0 & $-\mathrm{N}$ \\
\hline
\end{tabular}

The outputs of the circuit consist of a bit indicating the sign of the summed value (which is used to control the accumulator to add or subtract the value), and N/2 +1 bits for activating the multiplicand to be used according to the summed values. By using truth tables and Karnaugh maps, the decoding circuit can be easily derived.

For VCO and the D/A part of the tracking loop, no modification is needed except for some minor adjustment of the resistive trimmers.

\subsubsection{Possible Implementation on Xilinx 4000 FPGA Family}

A decoder with PN code length shorter than 127 and symbol size smaller than 3 can always be fit into the XC4010 FPGA chip. It is easily seen that the choices of the 2 factors, the PN 
code length and the symbol size are compromised when trying to fit the schematic into the FPGA with either the PN code length longer than 127 or the symbol size larger than 3 . The longer the PN code length, the smaller the symbol size can be decoded and vice versa.

It is very difficult and inaccurate to approximate the resource required for a decoder with different PN code length and symbol size combinations because the basic building block of a FPGA chip is a CLB which contains 24 input function generators and 2 flip flops. As discussed in Section 6.1, the function generators and flip flops in each CLB are not completely independent. The counting of $\mathrm{F}$ and $\mathrm{G}$ function generator involves the dividing a logic circuit into groups with a maximum of 4 inputs. The grouping is straight forward for a simple circuit like the tap select module in Chapter 4. For a more complex circuit like the early and late correlators, it is very tedious to divide the circuit into 4-input groups. The easiest way to count the resource used is to compile the circuit in Cadence system. The resource needed by the circuit is reported to show whether or not the circuit can be fitted into a particular FPGA. If a circuit requires too much resource to fit into the chip, one can either consider using a chip with more resource, or trim the circuit like the decision device discussed in Chapter 4.

\subsection{The Maximum Throughput of the FPGA Based Decoder}

For a CPSK decoder with PN code length $2^{\mathrm{M}}-1=\mathrm{G}$, symbol size $\mathrm{s}$, and chipping rate $\mathrm{R}_{\mathrm{c}}$, the data rate $R_{d}$ is given by:

$$
R_{d}=R_{c}\left(\frac{s}{G}\right)
$$

and the number of different code phases is $2^{\mathrm{s}}$, with the constraint that the phase shifts must be separated by at least 2 chips. By trying different s, G combinations, the maximum s/G ratio 
occurs when $s=2$ and $G=15$. From [19], the $\mathrm{XC4000} \mathrm{family} \mathrm{can} \mathrm{run} \mathrm{at} \mathrm{synchronous} \mathrm{clock} \mathrm{rates}$ of up to $60 \mathrm{MHz}$. The maximum $\mathrm{Rc}$ is therefore equal to $30 \mathrm{MHz}$ and the maximum data throughput $R_{c(\max )}$ is $4 \mathrm{M}$ bits/s. At this rate, the processing gain is 15 and the throughput is doubled as compared with conventional SS/DS signaling. Please note that the actual maximum clock should be checked with the maximum end-to-end delays compilation report after the design is compiled to ensure the circuit can be run at the maximum speed. 


\section{Chapter 7 Conclusions}

\subsection{Summary}

This thesis presented the design, implementation, and performance of a CPSK receiver. The receiver consists of 3 main parts, the IF demodulator, the CPSK decoder, and the PC interface. Priority in design was given to simplicity, modularity, and flexibility to decode with different parameters used in the CPSK signaling scheme. The key contributions in this thesis include solving the carrier and chip synchronization problems, and building the CPSK decoder that can be fit into a Xilinx FPGA chip.

To solve the carrier synchronization problem, a Costas loop consisting of a multiplier and a VCO was implemented in the IF demodulator to lock the phase of the LO to that of the carrier. However, there is still a 180 degree phase uncertainty which is resolved by the circuitry in the CPSK decoder using positive and negative thresholds.

The chip synchronization is done in 2 steps, acquisition and tracking. In acquisition mode, the phase of the local PN code slides in half-chip steps until it matches the code phase of the input signal to within half a chip difference. The system then switches to the tracking mode to decode data and to fine tune the phase alignment by employing a tracking loop. The switching between acquisition and tracking mode is governed by a search lock logic circuit employing a modified double dwell serial search scheme.

The CPSK decoder was designed to decode the CPSK signal with PN code length of 127 and symbol size of 3 . All the modules of the decoder, except a D/A converter and a VCO used to generate the master clock signal, was implemented in a Xilinx 4010 FPGA chip to minimize the 
number of components needed and ease system reconfiguration. The circuit was tested using Verilog Simulation before downloaded to the programmable chip. To eliminate the 180 degree phase uncertainty when demodulating the IF signal, extra circuitry consisting mainly of multiplexers is used to compare with 2 thresholds, in the cases when the phase of the demodulated is 0 and 180 degree shifted respectively. The decoder was found to decode the CPSK signal correctly.

The PC interface was built using discrete logic ICs and a FIFO memory chip so as to read the data from the CPSK decoder. Programs written in C were used to control the PC interface to read data and calculating the symbol error rate of the CPSK receiver.

To evaluate the performance of the receiver, AWGN noise and some jamming signal from another CPSK transmitter were injected into the communication channel for calculating the SER at different SNR and jamming level. After converting SER to BER, the measured BER vs. SNR curves with and without jamming are compared with those of an ideal receiver. It was found that there was a 0.25 to $0.85 \mathrm{~dB}$ of performance degradation when the carriers were perfectly synchronized during a BER measurement, and a further $1 \mathrm{~dB}$ degradation was measured when the Costas loop was used. Moreover, the addition of the jammer further degraded the BER performance and the decoder simply lost lock if the transmitter to jammer power ratio was lower than 6.3.

Since the decoder part of the CPSK Receiver is implemented in an XC4010 FPGA chip, the decoder can be easily configured to decode data with different symbol sizes and PN code length with some modifications on the original schematics. The possible symbol size / PN code length combinations and the outlines of modifications to each of the major components were also studied and presented. In the present design, the data throughput is relatively low because it is 
- limited by the length of the PN code and the symbol size used, and the chip frequency of the transmitter built with DSPs. However, for the Xilinx 4000 FPGA family, the maximum running clock frequency is $60 \mathrm{MHz}$. The maximum data rate that can be achieved by the decoder can be increased to $4 \mathrm{M}$ bits/s when a PN code length of 15 and symbol size of 2 are used.

\subsection{Future Work}

The thresholds used in the CPSK decoder were chosen based on the half of the maximum correlated value. They may not be the optimal ones when noise and jamming signals are used. To obtain a more optimal performance, the thresholds can be fine-tuned by further experimentation or theoretical analysis with the consideration of noise and jamming signal.

The CPSK decoder employs a modified double dwell serial search method with the 2 dwell times having the same length during acquisition. The acquisition time can be further shortened by reducing the first dwell time. This will involve the re-consideration of the timing problem and additional circuitry is needed.

The receiver was designed to decode CPSK signal with PN code length of 127 and symbol size of 3. Since the decoder is implemented in an FPGA, different versions of the decoder can be designed to form a hardware library so that CPSK signal with different code lengths and symbol sizes can be decoded. In this case, the PC interface should be designed so that different configuration files can be loaded into the FPGA chip.

The CPSK receiver can be applied and tested in a WLAN system. In that case, a suitable MAC layer protocol is needed to ensure reliable data transmission. IF/RF and RF/IF converters are also need in order for the system to work in an on-air environment. 


\section{Bibliography}

[1] R. C. Dixon, Spread Spectrum Systems with Commercial Applications, John Wiley \& Sons, Inc., 1994.

[2] Pickholtz et al., "Theory of Spread-Spectrum Communications", IEEE Trans. Comm., 30(5):855-884, May 1982.

[3] R. A. Scholtz, "The Spread Spectrum Concept", IEEE Trans. Comm., 25(8):748-755, Aug. 1977.

[4] A. Wong, Analysis of Techniques to Enhance the Performance of Direct Sequence Spread Spectrum Signaling for Wireless Data Communications, Master Thesis, Department of Electrical Engineering, UBC, July, 1995.

[5] R. Link, Design, Analysis, and Implementation of a DSP-BASED Modem for Code-PhaseShift-Keying, Master Thesis, Department of Electrical Engineering, UBC, October, 1996.

[6]. R. E. Ziemer and W. H. Tranter, Principles of Communications, 3rd. Edition, Houghton Mifflin Company, 1990.

[7] D. T. Magill, F. Natali, and G. Edwards, "Spread-spectrum Technology for Commercial Applications", Proc. IEEE, 82:572-584, Apr. 1994

[8] D. C. Kemdirim and JIM S. Wight, "DS SSMA with Some IC Realizations", IEEE Journal on Selected Areas in Communication, 8(4):663-674, May, 1990.

[9] M. M. Mano, Digital Design, 2nd Edition, 1991, Prentice-Hall

[10] L. W. Couch, Digital And Analog Communication Systems, 4th Edition, 1990, Macmillan Publishing Company.

[11] Mini-circuits, RF/IF Designer's Guide, Mini-circuitrs, 1996

[12] H. Wang, Spread Spectrum Modem IF Receiver, Technical Manual, Board Revision 1.0, June, 1995. 
[13] M.K. Simon, Spread Sprectrum Communications, Vols. 1,2,3, Computer Science Press, 1985.

[14] J. J. Jr. Spiker and D. T. Magil, "The delay-lock discriminator - An optimum tracking device", Proc. IRE, pp. 1403-1416, Sept. 1961.

[15] D. M. DiCarlo and C. L. Weber, "Multiple Dwell Serial Search: Performance and Application to Direct Sequence Code Acquisition", IEEE Trans. Comm., 31(5):650-658 May 1983 ,

[16] R. A. Yost, and R. W. Boyd, "A Modified PN Code Tracking Loop: Its Performance and Implementation Sensitivities", Rroc. Nat. Telecommun. Conf., Houston, TX, pp. 61.5.161.5.5, Dec. 1980.

[17] W. J. Gill, "A Comparison of Binary Delay-Lock Tracking-Loop Implementations", IEEE Trans. Aerosp. Elect. Syst., 2(4):415-429, July 1966.

[18] Texas Instruments, High-Performance FIFO Memories, 1994.

[19] Xilinx, The Programmable Logic Data Book, Xilinx Inc., 1994

[20] Cadence, Design Entry with Composer, Cadence Design Systems Inc., 1994

[21] Cadence, Digital Logic Simulation, Cadence Design Systems Inc., 1994

[22] Cadence, XACT Hardware and Peripherals Guide, Cadence Design Systems Inc., '1994

[23] L. C. Eggebrecht, Interfacing to the IBM Personal Computer, 2nd. Edition, Howard W. Sams and Company, 1994.

[24] T. Shanley and D. Anderson, ISA System Architecture, 3rd. Edition, Addision-Wesley Publishing Company, 1995.

[25] K. H. Yeung, A Course in Statistics and Probability, 3rd Edition, Youth Bookroom, 1985. 


\section{Appendix A. The Pin Assignment of the FPGA Decoder Chip}

The Xilinx 4010-PC84 FPGA chip is packed in a 84-pin PLCC package of which 61 pins can be used as either inputs or outputs. The other 23 pins are used for power and signaling pins during configuration. In this design, only 41 of the $61 \mathrm{I} / \mathrm{O}$ pins are used. The circuit for connecting the FPGA decoder to the 27256 EPROM used for programming, to the A/D converter from the IF demodulator, and to the PC Interface is shown in Appendix B. The pin assignments are shown in the following 2 tables. Table A.1 shows the pins that are actually used in the signaling and data paths while Table A.2 shows the pins that are used primarily as test points in different parts of the decoder circuit.

Table A.1 Pin Assignment For Control and Data Paths

\begin{tabular}{|c|c|c|}
\hline Pin \# & Name & Description \\
\hline 70 & $\mathrm{D}<0>$ & Bit 0 (LSB) of digital data input to the decoder \\
\hline 69 & $\mathrm{D}<1>$ & Bit 1 of digital data input to the decoder \\
\hline 68 & $D<2>$ & Bit 2 of digital data input to the decoder \\
\hline 67 & $\mathrm{D}<3>$ & Bit 3 of digital data input to the decoder \\
\hline 66 & $\mathrm{D}<4>$ & Bit 4 of digital data input to the decoder \\
\hline 65 & $\mathrm{D}<5>$ & Bit 5 of digital data input to the decoder \\
\hline 62 & $D<6>$ & Bit 6 of digital data input to the decoder \\
\hline 61 & $\mathrm{D}<7>$ & Bit 7 (MSB) of digital data input to the decoder \\
\hline 29 & CK & System Clock input (Twice to the chip rate) \\
\hline 50. & RS & Reset \\
\hline 47 & RS2 & Reset 2 (must be tied with RS) \\
\hline 5 & SYM_STR & $\begin{array}{l}\text { Strobe signal to write decoded data DATA_OUT }<2: 0>\text { in to } \\
\text { the FIFO of the PC Interface }\end{array}$ \\
\hline 39 & TRK_DIFF $<0>$ & Bit 0 (LSB) of the tracking error signal \\
\hline
\end{tabular}


Table A.1 Pin Assignment For Control and Data Paths

\begin{tabular}{|l|l|l|}
\hline Pin \# & \multicolumn{1}{|c|}{ Name } & \multicolumn{1}{c|}{ Description } \\
\hline 38 & TRK_DIFF $<1>$ & Bit 1 of the tracking error signal \\
\hline 37 & TRK_DIFF $<2>$ & Bit 2 of the tracking error signal \\
\hline 36 & TRK_DIFF $<3>$ & Bit 3 of the tracking error signal \\
\hline 28 & TRK_DIFF $<4>$ & Bit 4 of the tracking error signal \\
\hline 27 & TRK_DIFF $<5>$ & Bit 5 of the tracking error signal \\
\hline 26 & TRK_DIFF $<6>$ & Bit 6 of the tracking error signal \\
\hline 25 & TRK_DIFF $<7>$ & Bit 7 (MSB) of the tracking error signal \\
\hline 46 & SLOCK $<0>$ & Search Lock System State (MSB) \\
\hline 48 & SLOCK $<1>$ & Search Lock System State (LSB) \\
\hline 51 & DATA_OUT $<0>$ & Bit 0 (LSB) of decoded data \\
\hline 57 & DATA_OUT $<1>$ & Bit 1 of decoded data \\
\hline 79 & DATA_OUT $<2>$ & Bit 2 (MSB) of decoded data \\
\hline
\end{tabular}

Table A.2Pin Assignments For Testing Points

\begin{tabular}{|l|l|l|}
\hline \multicolumn{1}{|c|}{ Pin \# } & \multicolumn{1}{|c|}{ Name } & \multicolumn{1}{c|}{ Description } \\
\hline 35 & ADR<0> & Bit 0 (LSB) of the location of the reference correlator \\
\hline 4 & ADR $<1)$ & Bit 0 (LSB) of the location of the reference correlator \\
\hline 84 & ADR<2> & Bit 0 (LSB) of the location of the reference correlator \\
\hline 72 & CH_STATE & $\begin{array}{l}\text { Signal to change the state of the Search Lock Logic if } \\
\text { needed }\end{array}$ \\
\hline 19 & CHOICE_TH & $\begin{array}{l}\text { Signal to choose the upper threshold (thu) or lower threshold } \\
\text { (thl) for comparison }\end{array}$ \\
\hline 10 & CLK_OUT, & The clock signal to drive the PN code generator \\
\hline 14 & CLR_OUT & $\begin{array}{l}\text { Signal to clear the contents of the correlators at the end of } \\
\text { each correlation cycle }\end{array}$ \\
\hline 9 & COMPARE & $\begin{array}{l}\text { Signal to make comparison of the correlated outputs with the } \\
\text { thresholds }\end{array}$ \\
\hline 82 & GOUT0 & PN code faded into correlator 0 \\
\hline
\end{tabular}


Table A.2Pin Assignments For Testing Points

\begin{tabular}{|l|l|l|}
\hline \multicolumn{1}{|c|}{ Pin \# } & \multicolumn{1}{|c|}{ Name } & \multicolumn{1}{c|}{ Description } \\
\hline 83 & GOUT1 & PN code fed into correlator 1 \\
\hline 80 & HIT1 & Hit1 signal for the Search Lock Logic circuit. \\
\hline 81 & HIT2 & Hit2 signal for the Search Lock Logic circuit \\
\hline 8 & PSFT $<0>$ & $\begin{array}{l}\text { Bit } 0 \text { (LSB) of the location of the correlator that gives the } \\
\text { highest correlation }\end{array}$ \\
\hline 13 & PSFT $<1>$ & $\begin{array}{l}\text { Bit 1of the location of the correlator that gives the } \\
\text { highest correlation }\end{array}$ \\
\hline 7 & PSFT $<2>$ & $\begin{array}{l}\text { Bit 2(MSB) of the location of the correlator that gives the } \\
\text { highest correlation }\end{array}$ \\
\hline 41 & STORE & Signal to trigger various registers to store the values \\
\hline
\end{tabular}




\section{Appendix B. The Circuit for Configuring and Interfacing the FPGA Decoder Chip}

Every time when the system is powered up, the FPGA chip needs to be re-configured. This is done by attaching an EPROM that stores the configuration bit stream file to the chip. Since some of the I/O pins of the FPGA are used as address and data pins when reading the configuration file from the EPROM, data transceivers are used to separate the I/O pins connecting to the rest of the receiver and the data and address pins connecting to the EPROM. The circuit is shown in the Figure B.1. 


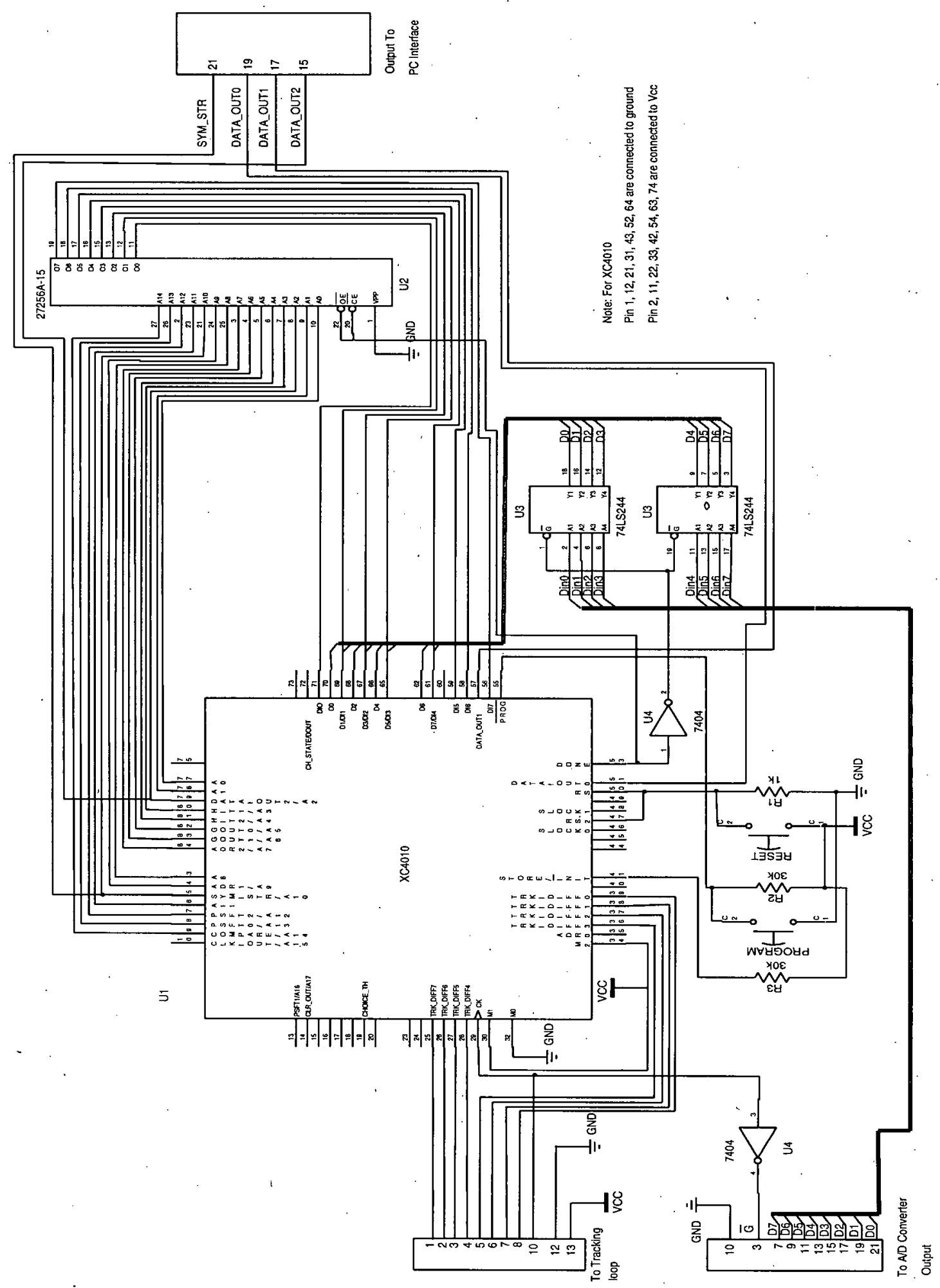

Figure B.1 Circuit for Interfacing the FPGA Chip 


\section{Appendix C. The Source Code for the PC Interface}

, The source code listed below is used for measuring the Symbol Error Rate (SER) of the CPSK Receiver when it is receiving data containing $0,1,2,3,4,5,6,7$ repetitive patterns. Programs for the reception of other data patterns were also written but since the source codes are identical except the function proTest(), they are not included in this appendix. The program prints the number of data symbols recevied and the number of errors accumulated for every 1000 symbols received.

PCI2.C:

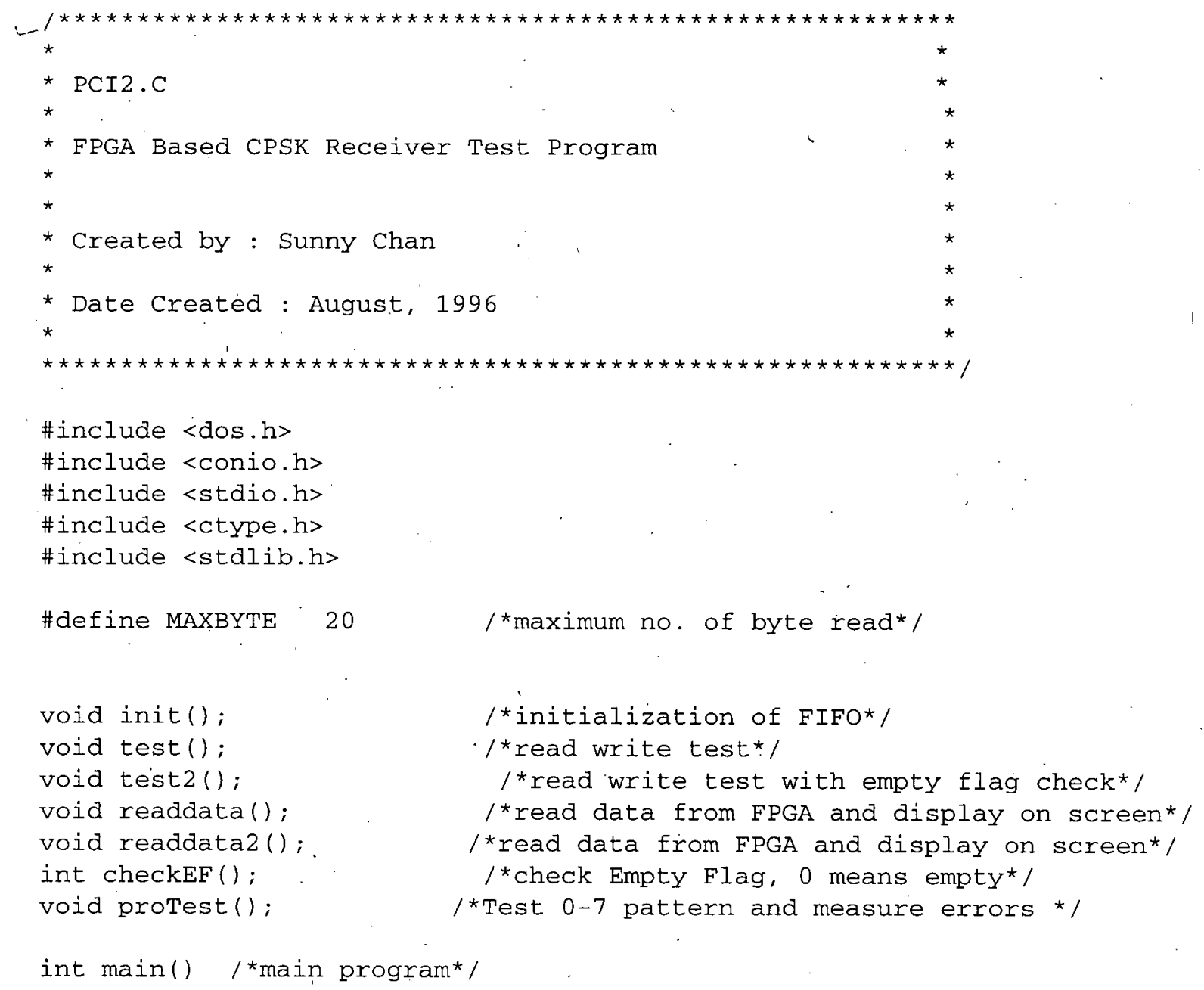




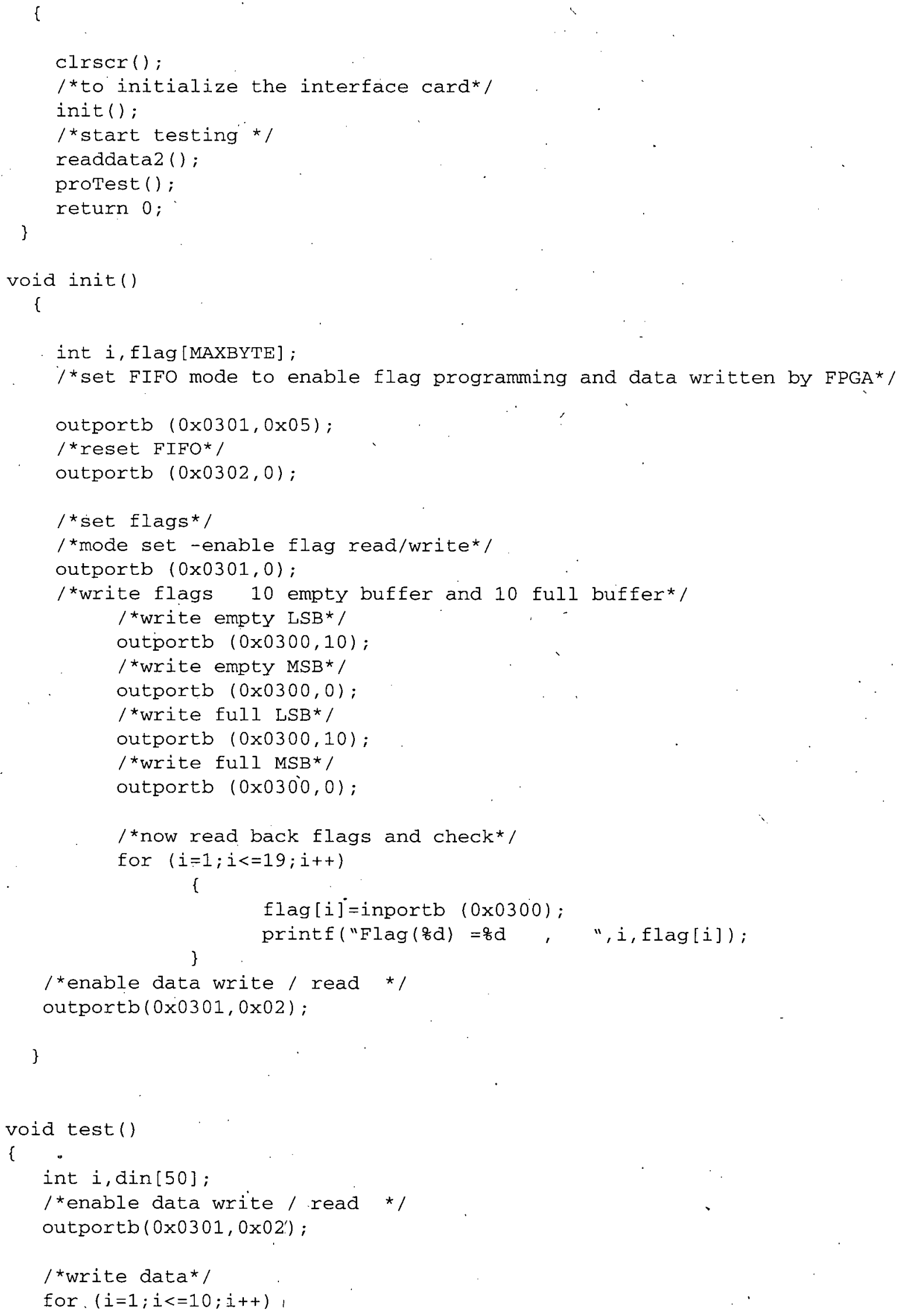




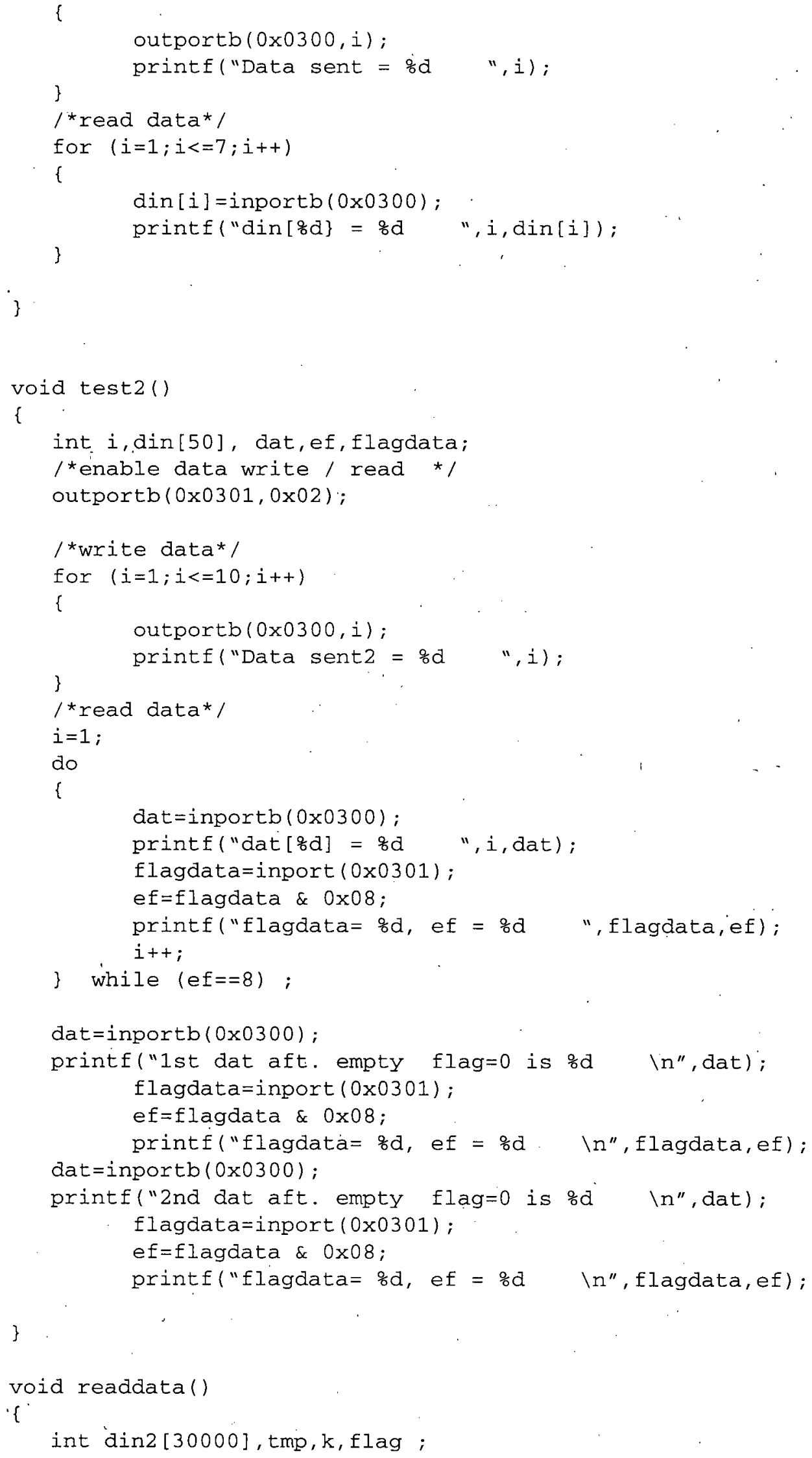




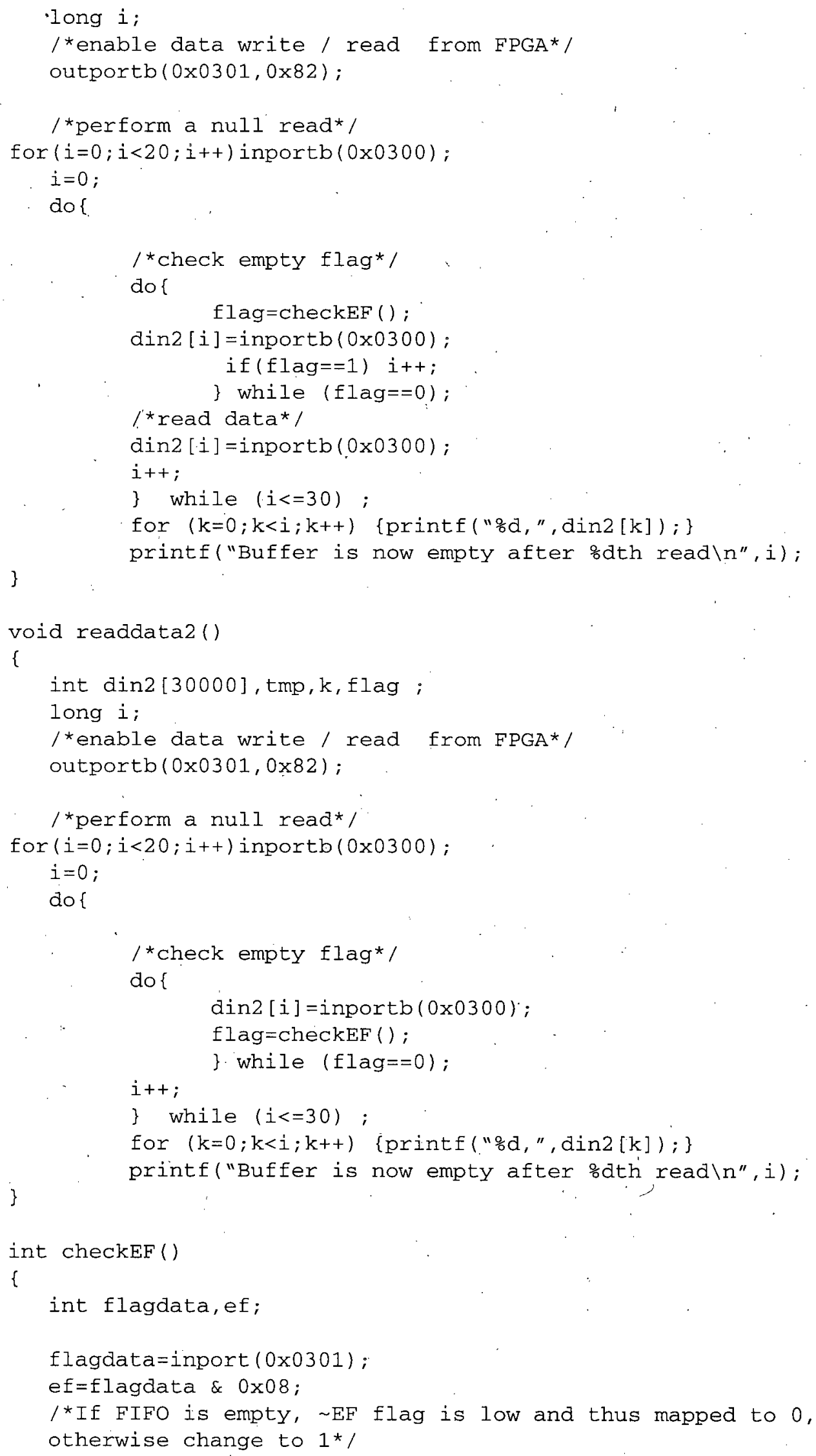




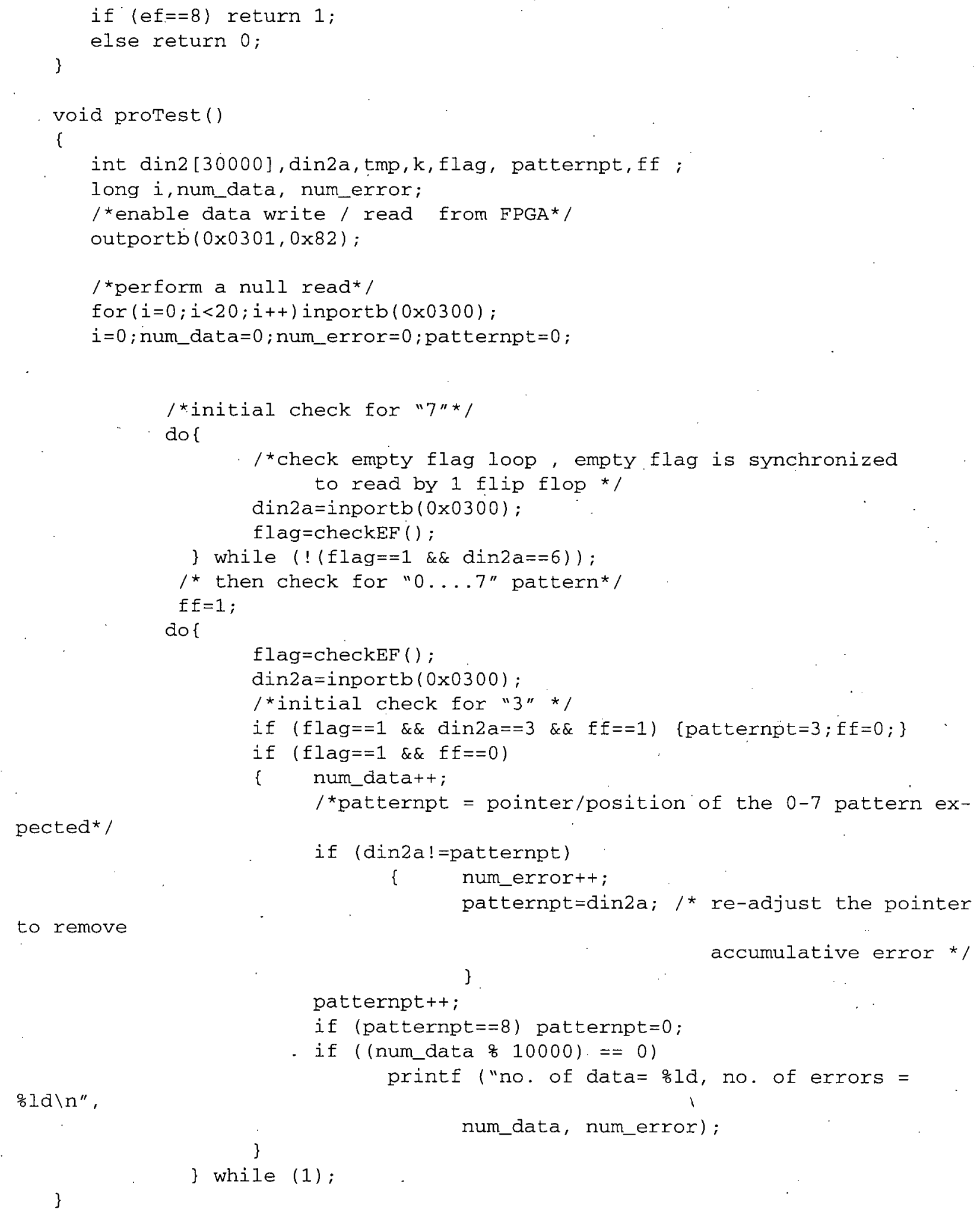

